\title{
WestVirginiaUniversity
}

THE RESEARCH REPOSITORY @ WVU

Graduate Theses, Dissertations, and Problem Reports

2010

\section{CFD applications in airship design}

\author{
Kevin Shields \\ West Virginia University
}

Follow this and additional works at: https://researchrepository.wvu.edu/etd

\section{Recommended Citation}

Shields, Kevin, "CFD applications in airship design" (2010). Graduate Theses, Dissertations, and Problem Reports. 2185.

https://researchrepository.wvu.edu/etd/2185

This Thesis is protected by copyright and/or related rights. It has been brought to you by the The Research Repository @ WVU with permission from the rights-holder(s). You are free to use this Thesis in any way that is permitted by the copyright and related rights legislation that applies to your use. For other uses you must obtain permission from the rights-holder(s) directly, unless additional rights are indicated by a Creative Commons license in the record and/ or on the work itself. This Thesis has been accepted for inclusion in WVU Graduate Theses, Dissertations, and Problem Reports collection by an authorized administrator of The Research Repository @ WVU. For more information, please contact researchrepository@mail.wvu.edu. 


\title{
CFD APPLICATIONS IN AIRSHIP DESIGN
}

\section{Kevin Shields}

\author{
Thesis submitted to the \\ College of Engineering and Mineral Resources \\ at West Virginia University \\ in partial fulfillment of the requirements for the degree of
}

Master of Science

In

Mechanical Engineering

\author{
Wade Huebsch, Ph.D., Chair \\ Gregory Thompson, Ph.D. \\ Gary Morris, Ph.D. \\ Department of Mechanical and Aerospace Engineering \\ Morgantown, West Virginia \\ 2010
}

Keywords: Airship, Computational Fluid Dynamics, Drag, Turbulence, Aircraft Design, Numerical Simulation, Fluent 


\section{ABSTRACT \\ CFD APPLICATIONS IN AIRSHIP DESIGN}

\section{Kevin Shields}

This thesis examines the aerodynamic characteristics and flight dynamics of a lighter-than-air airship using analytical and computational fluid dynamics (CFD). Modern airships have frequently been proposed for military operations; one of which, designated YEZ-2A, was a prototype whose design was initiated during the 1980's. Designs were evaluated through the wind tunnel tests of a 1:75 scaled model performed by Gomes. His tests relied on surface roughening techniques to compensate for the inability to maintain Reynolds number similarity among scales.

Later, the scaled prototype would receive further attention during a CFD study at WVU. This study was undertaken to numerically simulate the wind tunnel tests of Gomes on the scaled model. Current research was conducted subsequent to these investigations with multiple objectives. Most studies were satisfied by evaluating the prior scaled models in conjunction with full sized CFD models. These evaluations aided in validating the results of all three investigations while exploring potential errors in scaled modeling and turbulent studies. Goals included evaluating previous work through modern numerical analysis. This required a high fidelity CFD modeling approach with appropriate turbulent conditions. Sensitivity studies would ensure credible results independent of the approach. These conditions also served the second goal—providing a modern CFD approach to the design of airships.

This research validated the Gomes' data by means of CFD analysis of 1:75 scale and full scale models of the airship hull. Initial work reexamined the methods developed during the WVU study followed by their implementation for additional analysis. This research included axisymmetric temporal and grid sensitivity studies in addition to $3 \mathrm{D}$ analysis. This research confirmed that the techniques Gomes used were incapable of producing accurate full scale flight coefficients. Lastly, this research was presented as a design tool. It demonstrated a modern approach to the design and development of future airships via CFD analysis. 


\section{Acknowledgements}

First, I would like to thank my committee members, Dr. Wade Huebsch, Dr. Gregory Thompson and Dr. Gary Morris for the opportunity as well as their support. Thanks to Chris Menchini and Shanti Hamburg who were both vital contributors throughout my CFD learning experience. They were always ready and willing to provide me with some much needed assistance.

I would like to thank Dan Alkire, Spencer Clites, Travis Rowe and my brother, Ryan for their contributions. I need to thank my girlfriend, Stacy, for her continual support and encouragement. Thanks also to her mother, Dianne Mielecki. Her editing assistance was invaluable. Lastly, I must thank my parents, grandparents and the rest of my family for their constant love and guidance. 


\section{Table of Contents}

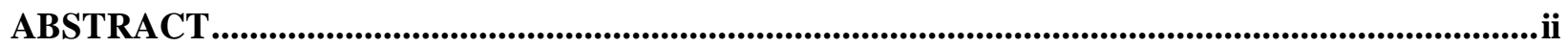

Acknowledgements ......................................................................................................................................................

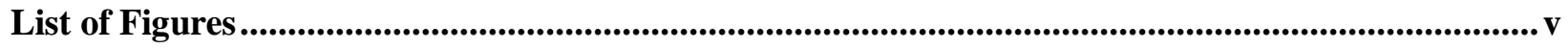

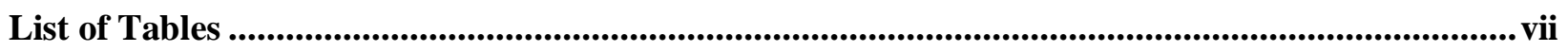

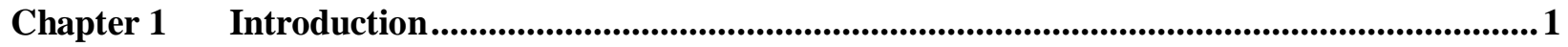

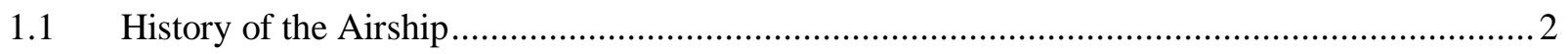

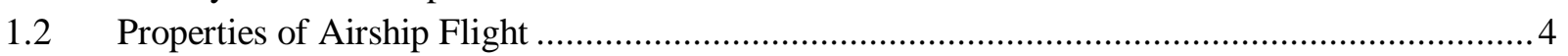

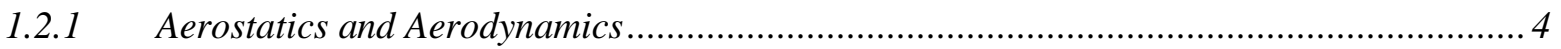

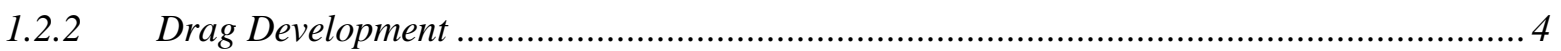

1.3 Theoretical, Experimental and Numerical Approaches .................................................... 8

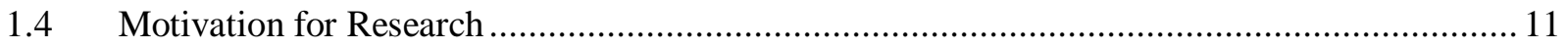

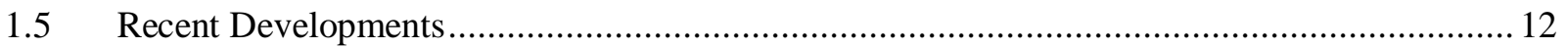

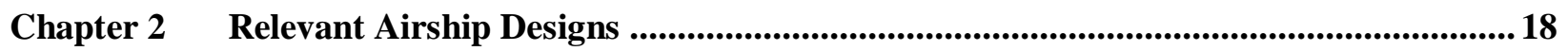

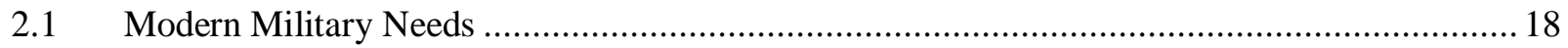

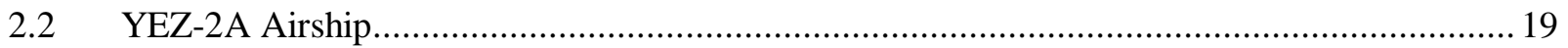

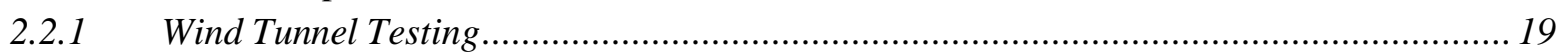

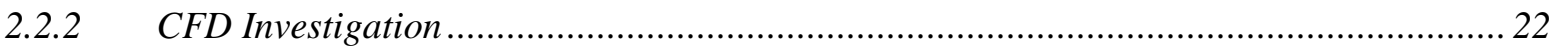

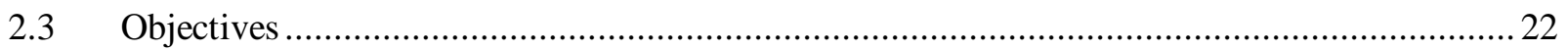

Chapter 3 Numerical Approach/Grid and Model Setup ...................................................................2 24

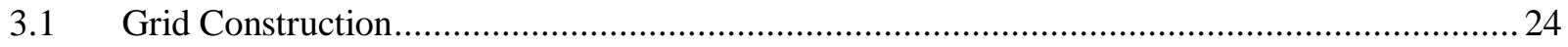

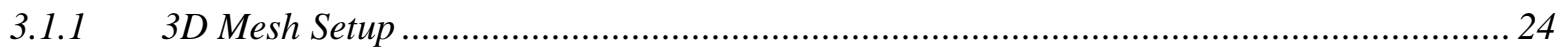

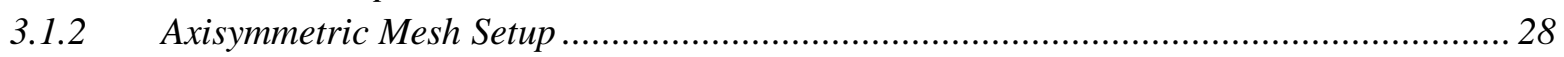

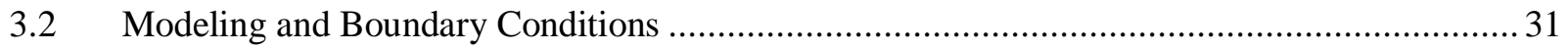

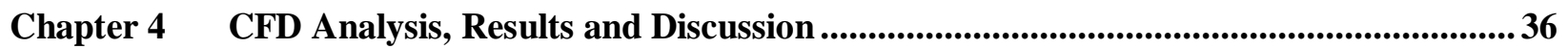

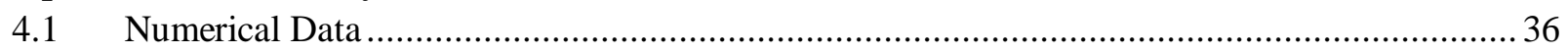

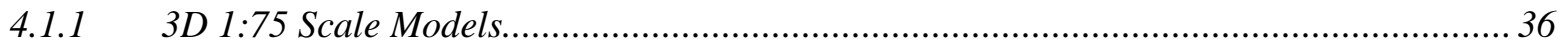

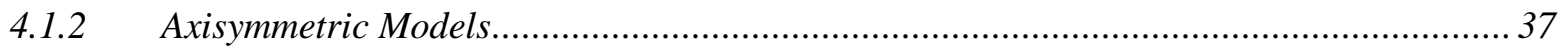

4.1.3 3D Full Scale Models .................................................................................................... 41

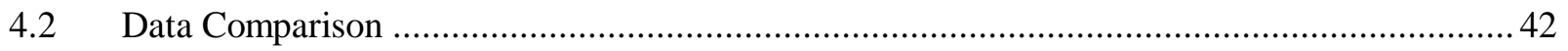

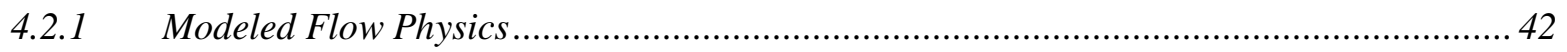

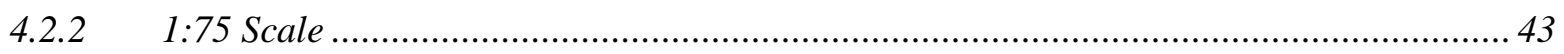

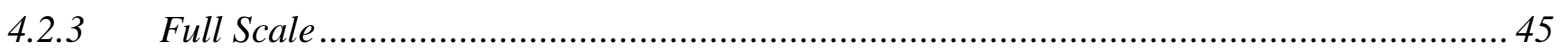

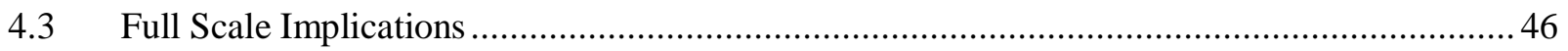

Chapter 5 Summary and Recommendations .......................................................................50

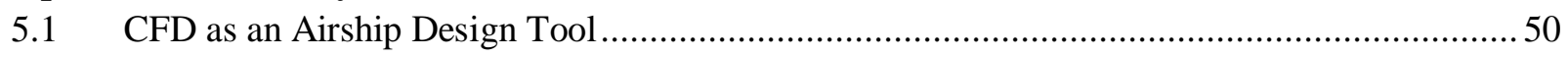

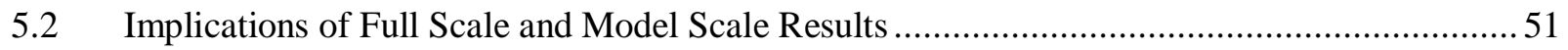

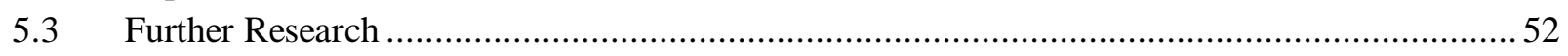

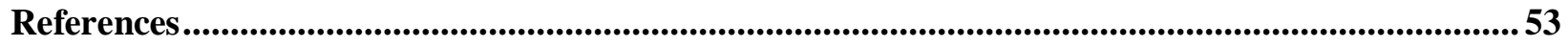

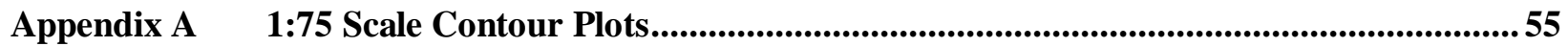

Appendix B Full Scale Contour Plots ......................................................................................................62 62

Appendix C Axisymmetric Investigations ........................................................................................69 


\section{List of Figures}

Figure 1-1: 1931 Demonstration Flight of Several U.S. Naval LTAs [7] .................................3

Figure 1-2: Streamlines of Mean Flow Showing Separation on a Spheroid at $30^{\circ}$ AoA [12] .......6 Figure 1-3: Transition of Flow Structure around a Cylinder with Respect to Reynolds Number [13] .8

Figure 1-4: Airship Volume as a Function of Pressure Height and Gross Weight [17] .............13

Figure 1-5: (Left) Aerocrane Scale Model Tested by the Navy and (Right) Heli-Stat Heavy-lift Vehicle [7].

Figure 1-6: Artist Rendering of HAA Concept and Corresponding Line of Sight Capabilities at 70,000 feet [17] 16

Figure 2-1: YEZ-2A General Features [4] ...................................................................... 19

Figure 2-2: Skyship 600 Full Scale (Left) and 1:75 Model Scale (Right) [4]........................21

Figure 2-3: YEZ-2A 1:75 Scaled Model in Wind Tunnel Test Setup Used by Gomes [4] .........21

Figure 3-1: Structured Hull Surface Mesh of 1:75 Scale YEZ-2A Airship Hull: (Top)

Longitudinal View and (Bottom) Angled View of Tail Mesh ....................................26

Figure 3-2: (Left) Unstructured Far-field Mesh Surrounding Airship Hull with Internal Mesh

Removed for Clarity and (Right) Horizontal Slice Showing Interior View of Total Mesh

Figure 3-3: (Left) Complete Near-field View and Near-wall Zoom View (Right) of the 3D

Airship Mesh ...................................................................................................28

Figure 3-4: Complete Hemisphere Grid Surrounding Airship Hull for Axisymmetric Models ...29

Figure 3-5: Zoomed Hull Region Showing Near-wall Grid of Course Mesh ...........................29

Figure 3-6: Axisymmetric Full Scale Grid with Zoomed Boundary Layer Region ....................30

Figure 3-7: 3D Model Overview with Boundary Conditions ...............................................34

Figure 3-8: Axisymmetric Model Overview with Boundary Conditions ................................35

Figure 4-1: Velocity Magnitude (m/s) Contour Plot of 1:75 Scale YEZ-2A Airship Hull at $10^{\circ}$ AoA

Figure 4-2: Lift Coefficient Comparison between 1:75 Scaled CFD and Wind Tunnel Models of the YEZ-2A and Skyship 600 Airship.

Figure 4-3: Drag Coefficient Comparison between 1:75 Scaled CFD and Wind Tunnel Models of the YEZ-2A and Skyship 600 Airship................................................................44

Figure 4-4: Lift Coefficient Comparison of 1:75 and Full Scale YEZ-2A CFD Models ............45

Figure 4-5: Drag Coefficient Comparison of 1:75 and Full Scale YEZ-2A CFD Models ...........46

Figure 4-6: Drag Coefficients Based on Wetted Area versus Reynolds Number for Several Airships with Various Fineness Ratios (1/d) [3] ........................................................47

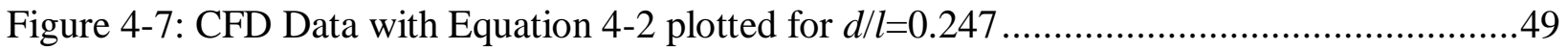

Figure A-1: Velocity Magnitude (m/s) Contour Plots of the YEZ-2A 1:75 Scale Hull-only CFD Model for Each Angle of Attack from the Longitudinal View .....................................55

Figure A-2: Pressure Coefficient Contour Plots of the YEZ-2A 1:75 Scale Hull-only CFD Model for Each Angle of Attack from the Longitudinal View ..... .56 
Figure A-3: Total Pressure (pascal) Contour Plots of the YEZ-2A 1:75 Scale Hull-only CFD Model for Each Angle of Attack from the Longitudinal View

Figure A-4: Pressure Coefficient Contour Plots of the YEZ-2A 1:75 Scale Hull-only CFD Model for Each Angle of Attack from the Longitudinal View ...........................................58

Figure A-5: Total Pressure (pascal) Contour Plots of the YEZ-2A 1:75 Scale Hull-only CFD Model for Each Angle of Attack from the Longitudinal View

Figure A-6: Shear Stress (pascal) Contour Plots of the YEZ-2A 1:75 Scale Hull-only CFD Model for Each Angle of Attack from the Longitudinal View....

Figure A-7: y+ Contour Plots of the YEZ-2A 1:75 Scale Hull-only CFD Model for Each Angle of Attack from the Longitudinal View

Figure B-1: Velocity Magnitude (m/s) Contour Plots of the YEZ-2A Full Scale Hull-only CFD Model for Each Angle of Attack from the Longitudinal View

Figure B-2: Pressure Coefficient Contour Plots of the YEZ-2A Full Scale Hull-only CFD Model for Each Angle of Attack from the Longitudinal View.

Figure B-3: Total Pressure (pascal) Contour Plots of the YEZ-2A Full Scale Hull-only CFD Model for Each Angle of Attack from the Longitudinal View

Figure B-4: Pressure Coefficient Contour Plots of the YEZ-2A Full Scale Hull-only CFD Model for Each Angle of Attack from the Longitudinal View.

Figure B-5: Total Pressure (pascal) Contour Plots of the YEZ-2A Full Scale Hull-only CFD Model for Each Angle of Attack from the Longitudinal View

Figure B-6: Shear Stress (pascal) Contour Plots of the YEZ-2A Full Scale Hull-only CFD Model for Each Angle of Attack from the Longitudinal View

Figure B-7: y+ Contour Plots of the YEZ-2A Full Scale Hull-only CFD Model for Each Angle of Attack from the Longitudinal View .68

Figure C-1: Wall y+ Values versus Axial Position (m) for Each Model and Grid Resolution .....69

Figure C-2: Dynamic Pressure (pascal) versus Axial Position (m) for Each Model and Grid

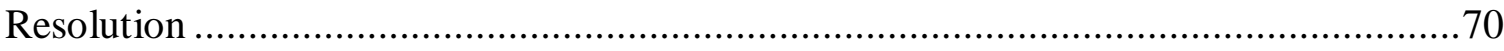

Figure C-3: Wall Shear Stress (pascal) versus Axial Position (m) for Each Model and Grid

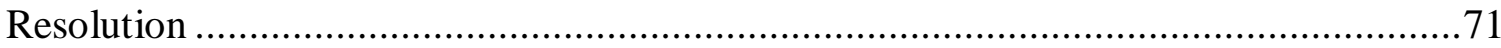

Figure C-4: Total Pressure (pascal) versus Axial Position (m) for Each Model and Grid Resolution

Figure C-5: (Top) Calculation of Original Time Step for Transient Analysis and (Below) Drag Coefficient Results for all Time Steps. .73

Figure C-6: Full Scale and Model Scale Transient Drag Coefficient for Each Time Step .74 


\section{List of Tables}

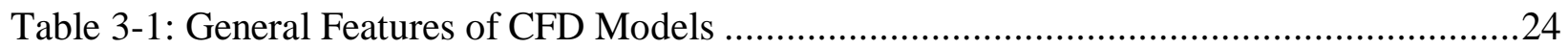

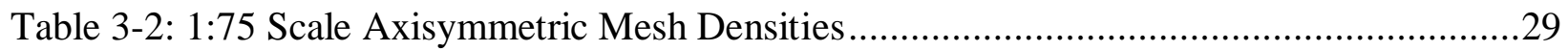

Table 3-3: Full Scale Axisymmetric Mesh Densities ..............................................................

Table 3-4: CFD Model Sensitivity Analysis Using the 1:75 Scale Hull with Fins Configuration

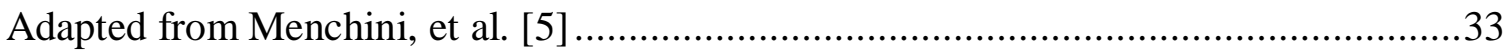

Table 3-5: Hull Dimensions and Critical Flow Parameters Applied to CFD Models ....................34

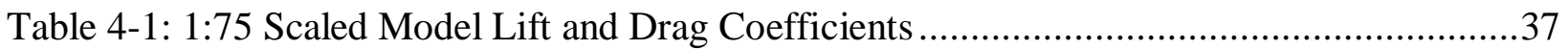

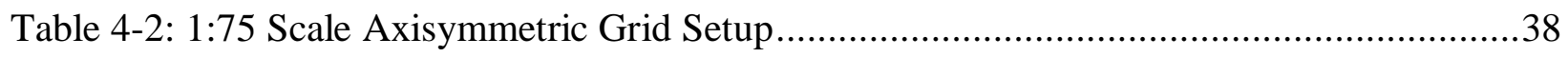

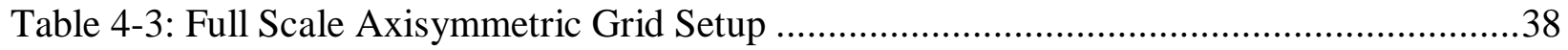

Table 4-4: 1:75 Scale Grid Convergence Results with Error Estimates ......................................39

Table 4-5: Full Scale Grid Convergence Results with Error Estimates........................................39

Table 4-6: Results of Transient Sensitivity Study Performed on Axisymmetric Medium Mesh

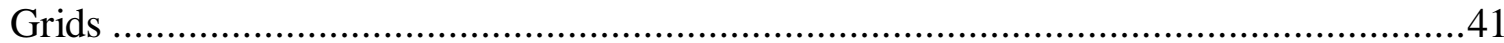

Table 4-7: 3D Full Scale Model Lift and Drag Coefficients....................................................42 


\section{Chapter 1 Introduction}

Aerodynamic forces that develop on an object arise from a complex and dynamic process that depends on a multitude of factors [1]. Each flow is essentially unique-developed specific to the object and conditions. For aircraft design much attention is directed towards the production of lift and drag forces. Attempts are made to minimize drag while generating enough lift to sustain flight. Airships and aerostats alike which rely on buoyancy to generate static lift see a major emphasis on drag reduction. Their shape is a major contributor to high drag values, though drag is dependent on a range of factors. Forces develop specific to the flow which can feature transitions, boundary layer separation, random vorticity ranging to the smallest of scales, and wake formation. All of these depend on their own range of factors and affect drag to a high degree. These conditions can combine to give such a complicated process that no complete theoretical understanding exists despite millennia of scientific research [2].

These intricate factors are essentially responsible for the aerodynamics of each object and the wealth of scientific considerations hint at their importance. Aircraft design must consider aerodynamic response to a variety of conditions and success depends on accurate predictions. With an absence of a theoretical solution, fluid dynamics evolved from experimental research to also include numerical approaches. Modern technology has given practical applications for numerical analysis. Digital computers are capable of modeling many flow scenarios with a high degree of accuracy. However, like the theoretical shortcomings before, numerical modeling is challenged when complex flows are encountered. It is now common to perform numerical analysis on aircraft using computational fluid dynamics (CFD). But most considerations are given to conventional aircraft designs while airships see less attention. The insight CFD provides is vast; it can give detailed and specific analysis never before possible. This modern numerical approach provides a valuable design tool to update the airship.

As always, it is imperative to validate any predictions. The lack of complete fluid theories meant many experiments were performed. In fact a physical understanding of fluidbody interactions developed from experimental observations. Until recent, scale modeling was the most common method for evaluating aerodynamics. Experimental data was useful for deriving semi-empirical equations for future predictions [3].

Successful scaled modeling requires an understanding of the physical processes that create drag. One should comprehend all individual features that contribute to the resulting flow. 
This understanding is beneficial in explaining complex scenarios encountered in many flows and a necessity for designers.

This thesis, therefore, considers the numerical analysis of a prototype airship using CFD with semi-empirical predictions and experimental data for validation. An attempt at explaining the physics involved in fluid flows is presented followed by a discussion on scaled model experiments of Gomes [4] and CFD analysis of Menchini, et al. [5]. A brief history of the airship is described prior to exploring aerodynamics. In summary, a thorough knowledge of the physical fluid mechanism is desired. This along with experimental evidence and available predictions allow for a successful approach to apply modern computer analysis to airship design.

\subsection{History of the Airship}

The origin of the modern airship can be dated to centuries ago. Before fixed wing aircraft pierced the skies man had already taken flight with the use of airships. Manned flight began with Lighter-than-Air (LTA) aircraft in 1783 when the hot air balloon was invented [6]. The balloon's flight capabilities relied on lift generated through buoyancy, which was created by heating air inside of the balloon. The balloon enabled man to first achieve flight.

LTA advances came in the $19^{\text {th }}$ century when propulsion systems were incorporated into balloons to provide thrust. Controlling these powered balloons required designers to introduce rudders and flaps to the aircraft. During this time the balloon's shape began to change as well. Envelopes changed from spheroid to ellipsoid designs creating a more streamlined body and with it the birth of the airship. These components still form the basis of the modern day airship. Today, objects like the balloon and airship which rely on buoyancy to generate lift are often referred to as aerostats.

Airship functionality was enhanced when internal combustion engines were introduced to efficiently replace human-powered propulsion. The $20^{\text {th }}$ century experienced a boom in the industry thanks to this new design. Almost immediately airships were used for military and commercial applications. Eruption of the first world war led several nations to use airships for reconnaissance and bombing missions. They proved rather ineffective at the latter but their success performing surveillance and communications was obvious.

Use continued following the war. Figure 1-1 shows several U.S. Navy aerostats during a 1931 demonstration [7]. The first transatlantic flights became commercially available on 
airships. Many also found use for expeditions and experiments. Airships returned to significant military use during the second world war. The U.S. Navy operated a fleet of 168 airships in antisubmarine roles to escort ships. Of the 89,000 ships escorted none were sunk, and only one airship would be lost to enemy fire [8]. Airship flight steadily declined following WWII as many nations phased out their use. Decline continued and in 1961 the Navy terminated its LTA program, placing the airship's future with the U.S. military in jeopardy [7]. Their use in other fields dwindled as well. Over the next four decades, the bulk of remaining airship operations would service the advertising industry.

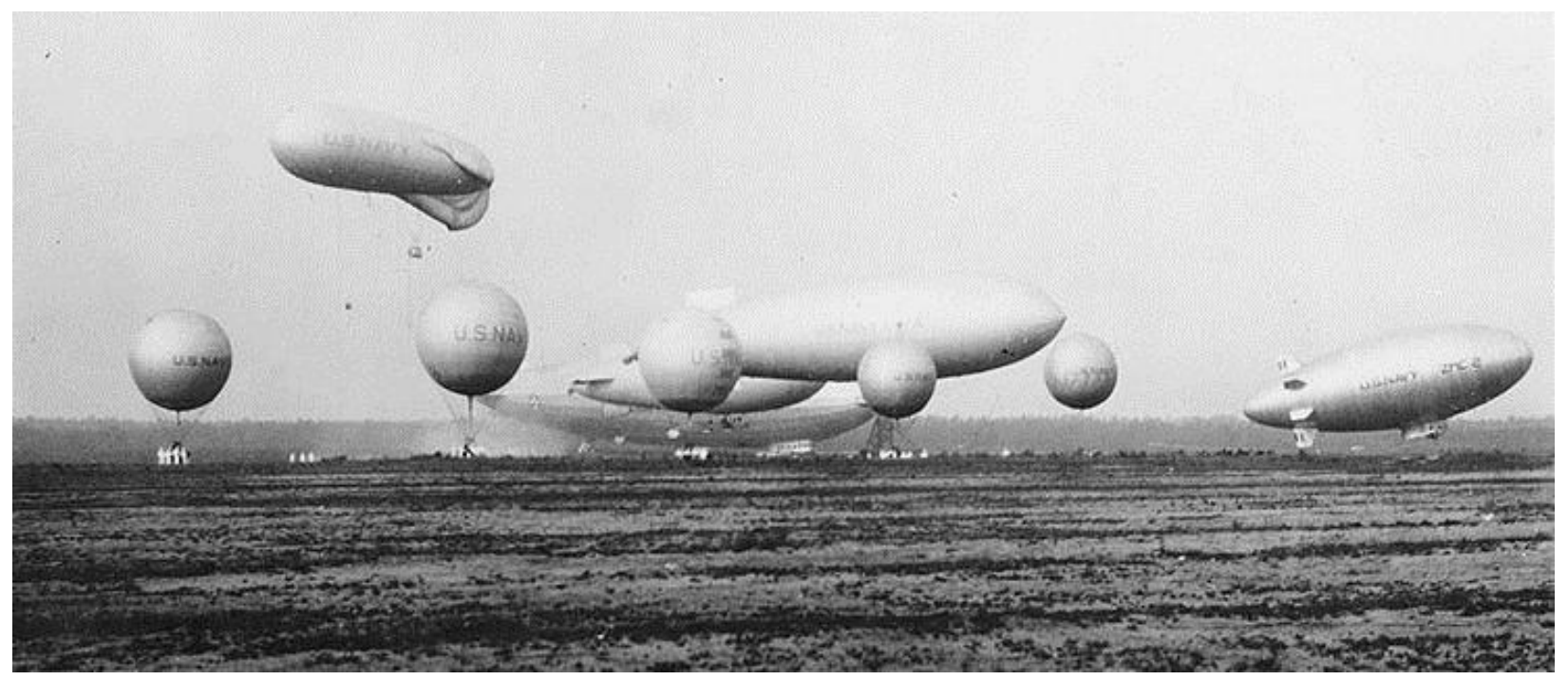

Figure 1-1: 1931 Demonstration Flight of Several U.S. Naval LTAs [7]

However, the vast military benefits offered by airships prompted revived interest decades later. Many experimental projects were undertaken beginning in the ' 70 s and ' 80 s. Ultimately these projects faced many setbacks that limited their success and airship use remained minimal. Although, they were successful in spawning a new interest into airships and the research yielded a wealth of data for future use. Over the past two decades airships have received more attention. The demand for research has increased and many previous studies see relevance once again. Present-day interest has continued to grow; airships are beginning to emerge in a variety of fields and their future appears promising. 


\subsection{Properties of Airship Flight}

\subsubsection{Aerostatics and Aerodynamics}

An aerostat is an aircraft which relies on the principle of buoyancy to sustain flight. A body immersed in the atmosphere is subjected to an upward buoyancy force equal to the weight of the displaced atmosphere. Every object surrounded by a fluid experiences this force. However, earth's atmosphere is composed of low density gasses that limit the generation of buoyant forces. For most objects any buoyancy force produced is greatly exceeded by the objects' weight. Therefore no lift is generated. This limit is what became the driving force for the design of aerostats and airships alike.

When the surrounding fluid is the atmosphere a large volume must be displaced to achieve an appreciable buoyant force. Upward lift is generated only when the weight of a body is lower than the buoyant force produced. Therefore, achieving static lift requires the object to displace a large volume of the surrounding atmosphere while weighing less than the atmosphere it displaces (i.e. Lighter-than-Air) [9]. Airships accordingly feature large lifting envelopes, or volumes, which pose many challenges to design and control.

Operating an airship in controlled flight posed one of the earliest aeronautical challenges. As a blunt object passes through air a large drag force develops that opposes the direction of motion, reducing the objects flight speed and hindering maneuverability. Constructing an object as a streamlined body will help reduce form drag and aid in the object's aerodynamic stability. Lutz, et al. [10] examined the non-linear behavior of forces and moments on an airship. Despite its streamline form "the bare hull shows an unstable aerodynamic moment up to very large angles of attack." The addition of control surfaces helps reduce instabilities. However, any additions to the hull affect drag in their own way and can complicate the flow process. There are many ways to alter the flow field, intentionally or not, which shapes the aerodynamic response. It is therefore important to understand each factor as it affects the drag development; the physical details of which follow.

\subsubsection{Drag Development}

Airship drag is generated from the hull and appendages (fins, engines, control car, etc.). "Due to its large size and volume, the bare hull can account for $60-70 \%$ of the total drag; this percentage increases with airship size as appendages become smaller in relation to the hull. 
Appendages affect drag due to their own resistance but also interfere with the flow over the hull" [9]. Developed flow is influenced heavily by interference effects arising from appendages or any surface imperfections. This dependency inhibits the separation of total drag into specific feature contributions on the airship. However, insight into flow structure can be gained with a physical understanding of the individual features that develop flow.

Interactions encountered between a submerged body and the surrounding fluid have been considered for hundreds if not thousands of years. One can argue that man was aware of the forces associated with such an interaction prior to recorded history when hunting spears were developed as slender streamlined objects. Early man may have been aware of the resulting forces but he lacked an understanding of the physical process that developed the flow. As time progressed differential calculus was introduced and theoretical predictions emerged. These early theories, which neglected viscosity, provided only a few exact solutions to fluid flow problems and their practical applications were limited. One example was the d'Alembert paradox. Published in 1752, it predicted that a body submerged in a non-viscous flow would have zero drag. This was obviously not true and was finally accounted for in 1904 when Prandtl introduced his boundary layer theory. Prandtl proposed the existence of a thin layer of fluid along a body's surface where viscous effects existed. This boundary layer theory would finally provide some answers to the question of how drag developed [11].

Centuries passed before scientists understood physical processes responsible for drag (or other aerodynamic forces) in a fluid-body interaction. Essentially, forces arise due to the transfer of momentum from a moving body to its surrounding fluid [3]. This exchange can develop drag forces on the body in two primary ways. First, the fluid offers some resistance due to friction generated when passing the body's surface. Secondly and depending on its shape, a high pressure region will develop in front of the object while a low pressure region develops in the rear, further resisting motion. These forces are labeled skin friction drag and pressure (or form) drag, respectively. Total drag on an object is usually a combination of these forces but it is highly dependent on shape. Drag on a thin flat plate parallel to a flow is almost entirely due to skin friction; however, pressure drag will dominate when it is perpendicular to the flow. A bluff body will therefore generate most of its drag through pressure drag. Streamlining the body, like an airship hull, will reduce the pressure drag but increase skin friction drag. 
Viscous effects are responsible for developing skin friction drag forces. These forces result from a shearing action in the flow that forms the boundary layer along a body's surface. Reduced fluid velocity in this region results in a loss of momentum which generates the drag force [9]. Additionally, drag can develop on a body in other ways. Frictional boundary layer losses may contribute to drag even more when the boundary layer separates from the surface of the body. If local fluid velocity is reduced enough in the inner portion of the boundary layer, conservation laws require the fluid to travel outward from the surface. This causes the boundary layer to separate from the surface of the object forming a wake region downstream. The separation affects static pressure on the object's surface within the wake region and contributes to pressure drag. This phenomenon will occur wherever the boundary layer thickness and adverse pressure gradients are large enough, usually along the rear side of a body [3; 9]. Figure 1-2 illustrates these effects on a prolate spheroid. A time-averaged flow pattern is visualized on the model showing streamlines as they converge towards the aft of the body before separating to form a wake [12].

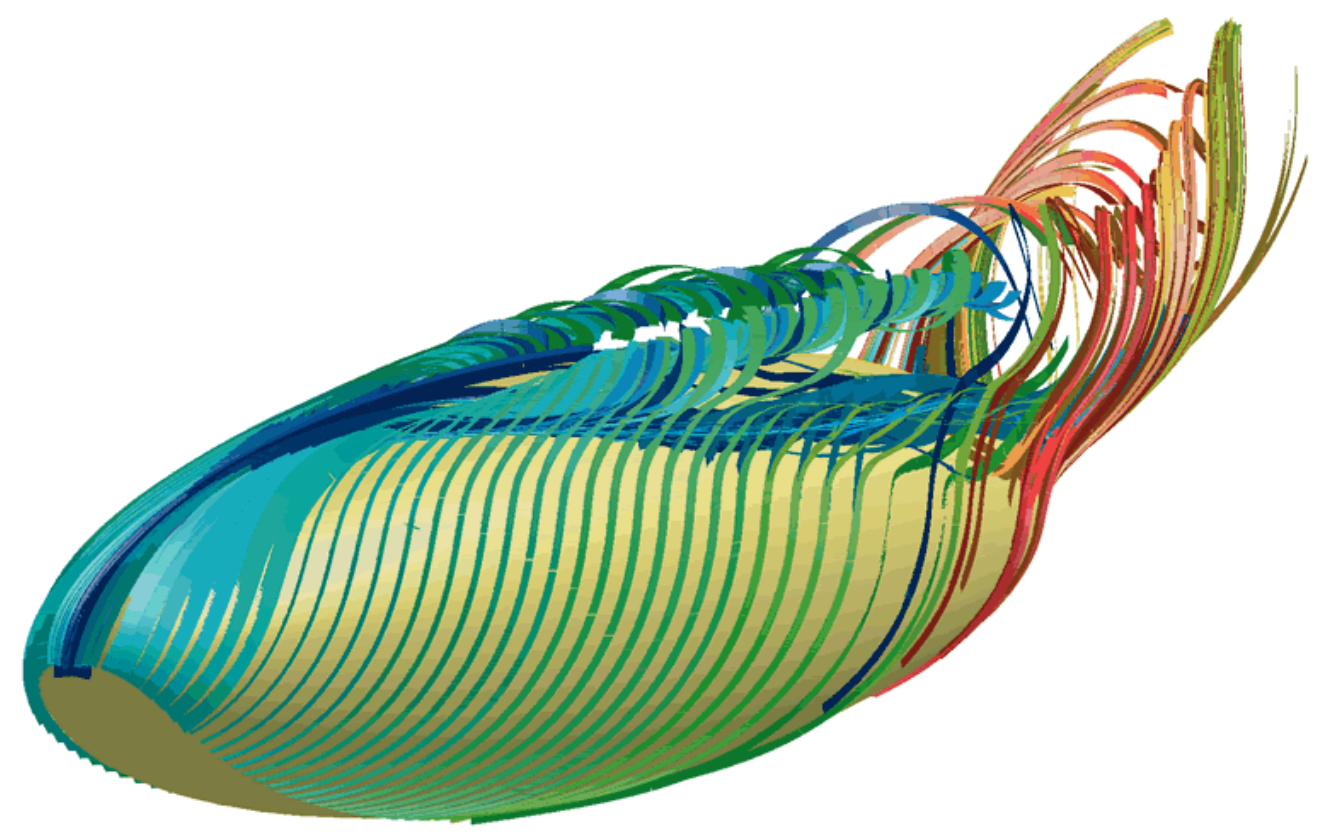

Figure 1-2: Streamlines of Mean Flow Showing Separation on a Spheroid at 30 ${ }^{\circ}$ AoA [12]

The separation line is highly dependent on the angle of attack (AoA). Inclined angles will also produce a lift force on the body and can induce an additional drag, termed induced drag. This occurs when surface pressures are not symmetrical on upper and lower surfaces of a body, 
as is the case for dynamic lift. The effects on a body inclined at an angle to the free stream include an increase in flow velocity along the upper surface and a reduction in velocity along the lower surface. This dynamic process forms a new pressure distribution that generates lift and some drag. The altered pressure distribution can affect boundary layer development, which in certain cases can be highly influential on the other drag forces [9].

In summary, drag forces can arise through various means. Explaining how these forces develop was not possible until viscosity effects were considered. Boundary layer development is influenced heavily by the pressure gradient along the body's surface and is foremost responsible for the generation of drag. Overall aerodynamic forces result from a complex process and exhibit a strong non-linear dependence on the condition of flow. Drag is related to an object's velocity but also depends on many other factors.

The Reynolds number, which relates inertial forces to viscous forces non-dimensionally, is often used to characterize fluid flow past an object. Effects of the Reynolds number on the flow around a cylinder are show in Figure 1-3. As the Reynolds number is increased beyond a specific stability limit the boundary layer flow transitions from laminar to turbulent flow. In the turbulent regime the flow eddies due to oscillating velocity components in a random nonlinear process that exchanges momentum. Transition often begins with a wave motion generated in the laminar boundary layer whenever dynamic flow forces dominate viscous forces. These waves can be "excited by surface roughness, through mechanical vibrations, by sound waves, or because of the turbulent oscillations in the artificial stream of a wind tunnel" [3]. When boundary layer flow along a body transitions to the turbulent regime the effects are witnessed in the resulting flow physics. This phenomenon induces greater shearing stresses leading to an increase in viscous drag. 


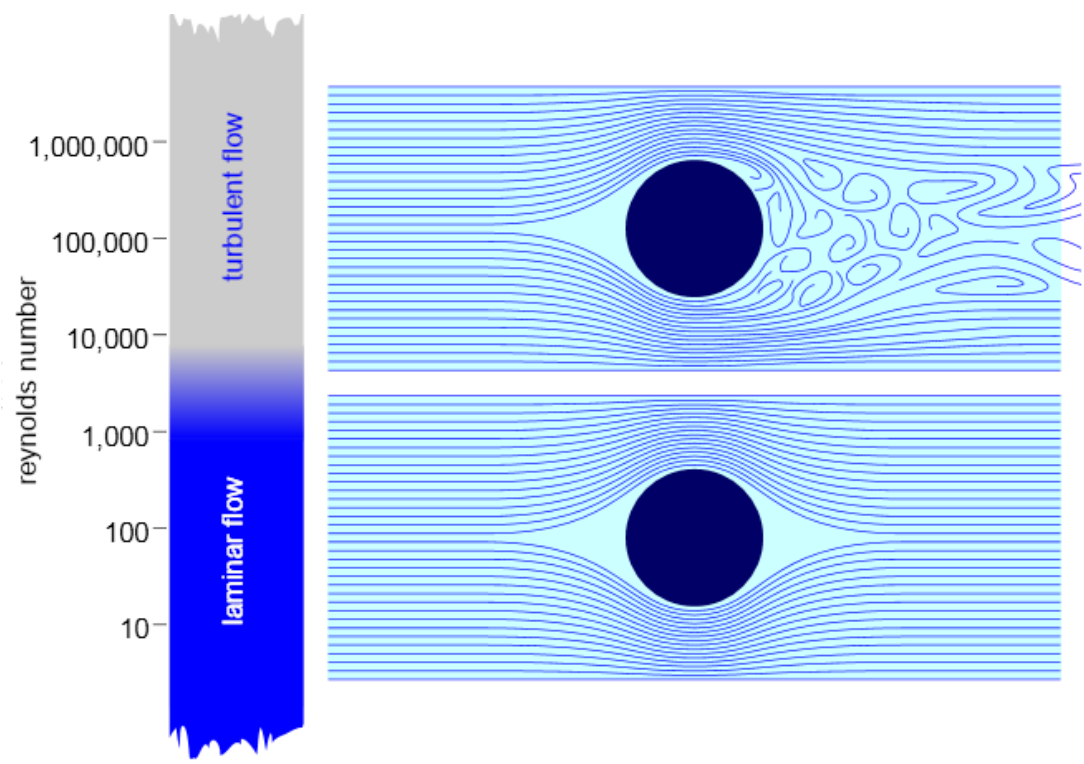

Figure 1-3: Transition of Flow Structure around a Cylinder with Respect to Reynolds Number [13]

A boundary layer transition point may occur earlier along a body when surface imperfections are present. This scenario can be highly influential on drag generation; this is often the case in reality and is sometimes unavoidable for full scale aircraft. For other circumstances surface imperfections are deliberately introduced. Manipulating an object to influence the location of a boundary layer transition can be of practical use and is often achieved by creating a rough surface. The effects of turbulence can delay boundary layer separation, which lowers the amount of pressure drag. Ultimately, under certain conditions the total amount of drag on a body can be reduced when a turbulent boundary layer is present.

\subsection{Theoretical, Experimental and Numerical Approaches}

All of the aforementioned effects can be experienced by an airship in flight, and are hence responsible for the aircraft's aerodynamics. It would be very beneficial to have a reliable means for calculating aerodynamic forces. However, each interaction contributes to an immensely complex flow structure, hindering attempts at theoretical predictions. Some situations do, however, match some limited theoretical solutions, such as the prediction of induced drag or use of boundary layer theory to predict separation. All in all, available pure theories see few practical applications for 3D flow around an object. These theoretical limitations forced early researchers to rely on scaled model experiments for analysis. Semi- 
empirical equations subsequently developed, like that of skin-friction drag coefficient, providing additional analysis and future predictions [3].

Scaled testing (e.g. scaled model in a wind tunnel) must preserve certain non-dimensional groups between model and prototype. Airships require subsonic wind tunnel modeling and it is usually the Reynolds number based on hull length that must be maintained. A consistent Reynolds number will allow direct comparisons between force coefficients of different sized but identically shaped models. However that can be a difficult task, so alternate techniques must sometimes be used. "It has long been established that the most important objective is to ensure that the transition from laminar to turbulent boundary layer occurs at about the correct position on the model" [9]. With this in mind the benefit of introducing turbulence through a rough surface or other means becomes evident. Skin friction coefficient is altered by a rough surface but form drag and lift are preserved. Since skin friction dominates airship flight a considerable error can arise; great care must be given to find the appropriate level of surface roughness.

Correlating results from small scale tests to full scale can be done in proportion to skinfriction drag. For bodies of revolution like an airship, drag associated with roughness and appendages is essentially constant with regard to Reynolds number. It is then possible to assume total drag is composed of two components. One constant drag component and another that varies in proportion to turbulent skin-friction drag coefficient as described by Hoerner. However, "the theoretical analysis of turbulent skin-friction drag is complex" [3]. No exact solution exists, but statistical functions from experimental data are available. They are semi-empirical in nature but provide practical solutions for turbulent skin-friction drag. As more and more data became available methods were refined to provide more accurate predictions.

As technology advanced, complex flows were increasingly numerically analyzed through computational fluid dynamics (CFD). Numerical analysis is one method that avoids potential problems associated with wind tunnel testing (e.g. scaling effects due to different Reynolds number). In addition, "the interesting parameters including the geometry can be changed very easily, and flow details can be investigated better compared to experiments" stated by Jakobi, et al. [14]. However, modeling the true fluid dynamic effects proves difficult, especially for three dimensional (3D) turbulent flows and worse yet for boundary-layer separation.

The Navier-Stokes (N-S) equations are considered to be the governing equations of fluid flow. They exist as a set of nonlinear and non-unique partial differential equations. They can 
provide a complete mathematical description for a variety of flows. However, no general analytical scheme exists to solve the equations, requiring individual evaluations for each problem [15]. The complex nature of the equations makes exact solutions difficult to obtain, and most are due to simplifications.

Many solutions can be approximated using numerical techniques. Partial differential equations are replaced with algebraic approximations and applied at a finite number of discrete mesh points throughout the continuous fluid. Equations are then solved over the entire mesh to simulate flow. Many laminar flows can be simulated to a near-exact degree, but even basic two dimensional flows may require hundreds of calculations and significantly more for three dimensions. Therefore the practicality of numerical modeling emerged in conjunction with development of the digital computer [11].

Under certain restrictions, numerical techniques allow for the simulation of many flows with accurate results. Equations can be directly solved for most laminar flows. However, success for turbulent flows requires all turbulent fluctuations to be modeled down to the smallest of scales in the computational mesh. Accordingly, as Reynolds number increases, the required mesh resolution increases as well, demanding greater computational resources. The fact that turbulent fluctuations can exist at such small scales like the Kolmogorov microscale, limits this method to the lowest of Reynolds number flows. Solving turbulence in this manner is termed direct numerical simulation (DNS) and requires so many numerical operations that most flows cannot be modeled even on today's most powerful computers, [11]. Turbulence is generally approximated using explicit models, instead of DNS. Most of these approaches like the RANS (Reynolds-averaged Navier-Stokes) modify the Navier-Stokes equations, separating the turbulent fluctuating property from its time-averaged value. These approaches require a turbulence model to introduce the fluctuations. Numerous models are available and they determine their values using various relations. Other methods are also available to simulate turbulent flows; each with their own advantages. Modern computational resources allow for turbulent analysis with advanced models and sophisticated CFD software. With a suitable model and setup, users can model turbulent flows with reasonably accurate results. Their capabilities do, however, diminish as more complexities are introduced into the flow field such as boundary layer separation. Usually these phenomena are not accurately modeled [16]. 
The present capabilities of CFD analysis provide a high level of aerodynamic insight, but it cannot provide the full details of complex turbulent flows. As technology progresses and computers advance, turbulent models may become more capable of simulating these interactions. CFD is a continually evolving field; it is important that turbulent analysis is performed with experimental and theoretical studies to counter these complex limitations.

\subsection{Motivation for Research}

Aerodynamic forces that arise on an airship are often the result of a complex three dimensional flow process. They exhibit a non-linear dependence on angle of attack and result in an unstable moment for most configurations. Flight mechanics are therefore extremely important in airship design. Non-linear aerodynamics require designers to consider a range of flow conditions and also prevent an analytical solution to the equations of motion. Researchers were forced to rely on experiments; using empirical and semi-empirical methods to determine the aerodynamics in question. Methods were improved upon and refined as more experiments and test data became available. Their use in aircraft design increased but they were still simplified methods and could not provide details of the specific flow conditions.

Obtaining more details of complex flows can be accomplished using a numerical approach. Computing solutions of the Reynolds-averaged Navier-Stokes equations is one form of the numerical analysis of CFD. This method is only possible thanks to today's powerful digital computers as it requires an enormous amount of computations. Difficulties do arise when highly complex flows are encountered, but overall a detailed amount of information can be obtained through the modeling process. Pressure distributions, boundary-layer development, separation, and more can all be observed using CFD analysis. Aerodynamic data for a range of flight conditions can now be obtained in greater detail through the numerical analysis process [10].

It is these reasons that lead to present research. Ideally a designer would like an aerodynamic data base of the resulting flow field for each design. CFD methods can provide a complete and detailed model of each flow to build such a data base. But it is beyond the scope

of this research to do so. Instead the focus lies on creating a CFD modeling approach to obtain force coefficients comparable to available wind tunnel data. The study uses the experimental 
data along with empirical predictions for validation purposes. This research also furthers past investigations into a prototype airship and will aid by applying modern analysis to its design.

\subsection{Recent Developments}

Throughout history the airship's role for military use has been a reoccurring development. Even though the prominent WWI airship era has passed, a modern use for these aircraft still exists. In recent times the airship became all but obsolete, with the few remaining in use for advertising purposes. However, their use in the military is again being considered.

While the development and technology of the airship was surpassed by that of fixed wing aircraft long ago, airships can still offer numerous advantages. Since airships do not rely on dynamic lift to stay aloft they can operate on a much smaller fuel supply. This allows for a highly efficient aircraft with great endurance. Static lift also allows the airship to maintain a stationary position, providing a noise free and stable aerial platform. Airships usually operate within a range of altitude up to 8,000 feet, but limits are unique for each design and operating environment. As an aerostat ascends there is a corresponding drop in atmospheric pressure. Gas inside the envelope will then expand until reaching a certain altitude, termed the pressure height. Further ascension requires lifting gas to be vented or a pressure differential will develop across the skin of the envelope causing additional stresses. Operating limits are usually selected below the pressure height unless the envelope is designed to handle these stresses. Altitude capabilities therefore depend on volume, temperature, and other design factors. A large lifting-gas volume is necessary for higher payloads but has a low pressure height, reducing with increasing ambient temperature. Minimizing weight for a given volume will allow for a maximum pressure height [9]. The relation of pressure height with respect to total system weight and airship volume is shown in Figure 1-4 at Mean Sea Level conditions (MSL). This indicates that "volume increases exponentially with ceiling, and linearly with weight" [17]. At higher altitudes the required volume becomes significantly larger for even small increases in weight. With this in mind airships have been structurally designed to deal with additional stresses developed while exceeding their pressure height. They can easily ascend to 20,000 feet and are considered for high altitude operations of up to 70,000 feet. 


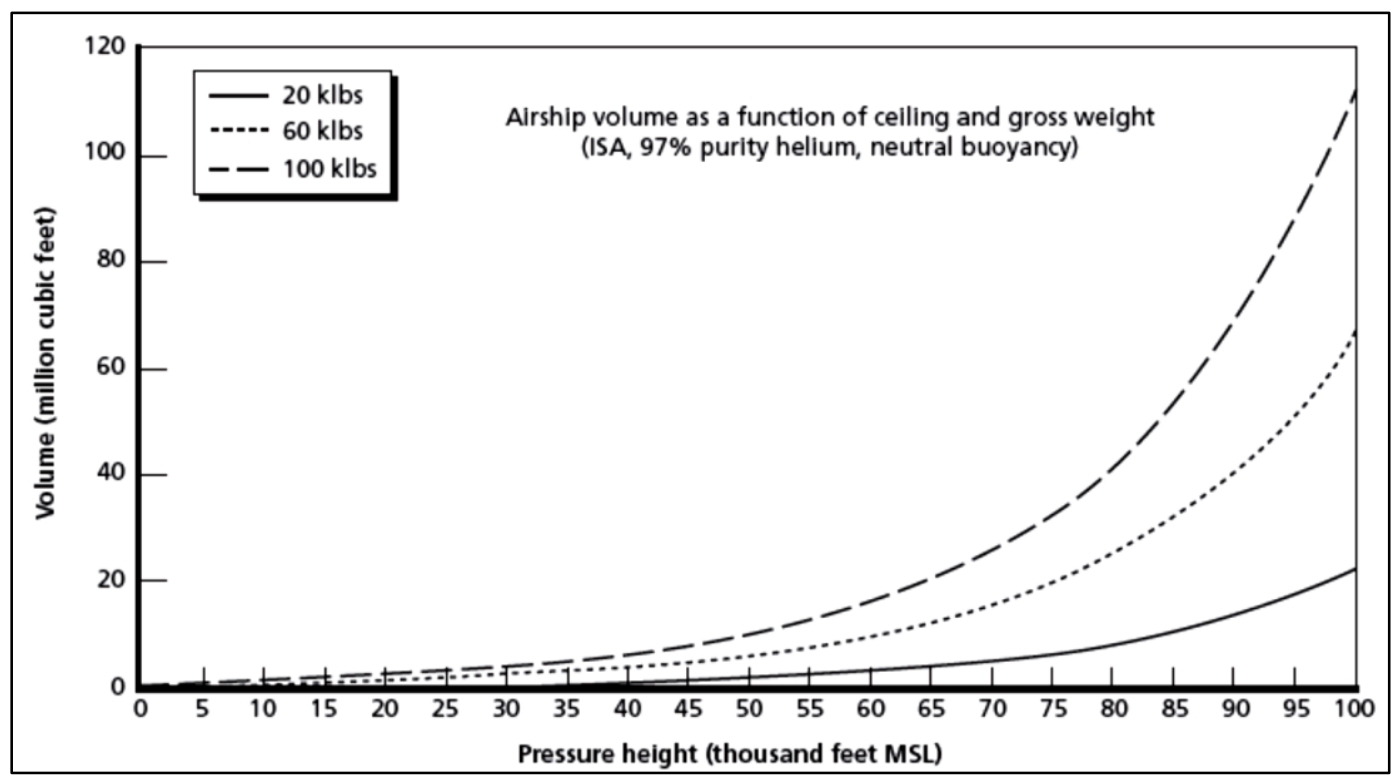

Figure 1-4: Airship Volume as a Function of Pressure Height and Gross Weight [17]

In summary, the airship and its distinct aerial properties make it an ideal aircraft for a number of roles. Arms treaty verification, search and rescue, border patrol, communications, surveillance, pollution monitoring, fire patrol, disaster assessment, use as a research platform, are all applications well suited for a modern airship [18].

The last four decades have witnessed a resurging interest in airships. The Navy-despite terminating its Lighter-than-Air (LTA) program just a decade before-once again pondered the potential benefits of a modern airship. The Coast Guard and NASA followed suite, leading to feasibility studies and radical new designs for the airship. Other government bodies also saw a potential so several LTA programs were funded. Many projects would put the airship in unconventional roles while others would revive past use with modern advances. Some unusual designs introduced a 'hybrid' airship, which combined features of an airplane or helicopter with an airship. One example began in 1974 when the Navy began the Aerocrane project. It was a spherical helium envelope rotated on its vertical axis with rotors and fins to generate vertical lift. The Aerocrane was intended for short distance, heavy load lifting. In 1980 another contract called for a heavy-lift airship for the U.S. Forest Service. This hybrid, called the Heli-Stat, featured a conventional hull paired with four rotors beneath [7]. Other programs, such as the Navy's Patrol Airship Concept Evaluation (PACE), assessed a conventionally designed airship outfitted with advanced sensors and modern equipment. They evaluated surveillance and communication benefits to military operations, one of the earliest and most successful roles for 
the airship. Unfortunately, none of these military projects would come to complete fruition. Some were evaluated as scaled modes, like the Navy's Aerocrane pictured in Figure 1-5. Others ended as operational prototypes like the Heli-Stat pictured as well. Some progressed to full scale flight testing like airships used in the Navy's PACE study. All in all, the last of the military programs were halted in the early 1990's for a variety of reasons. The concepts did succeed in other ways. They spawned a surge of interest into airships and drew consideration from other fields. Tethered aerostats were considered for a radar system to provide an air defense and drug enforcement network. In fact, eight sites have been in operation since 1980 along U.S. borders [17]. In addition, the studies generated an immense amount of data from wind tunnel modeling, test flights, and computer simulations. These all helped to provide modern analysis as well as upgrades to materials, equipment and construction. In whole, these projects did foster success for some LTA operations.

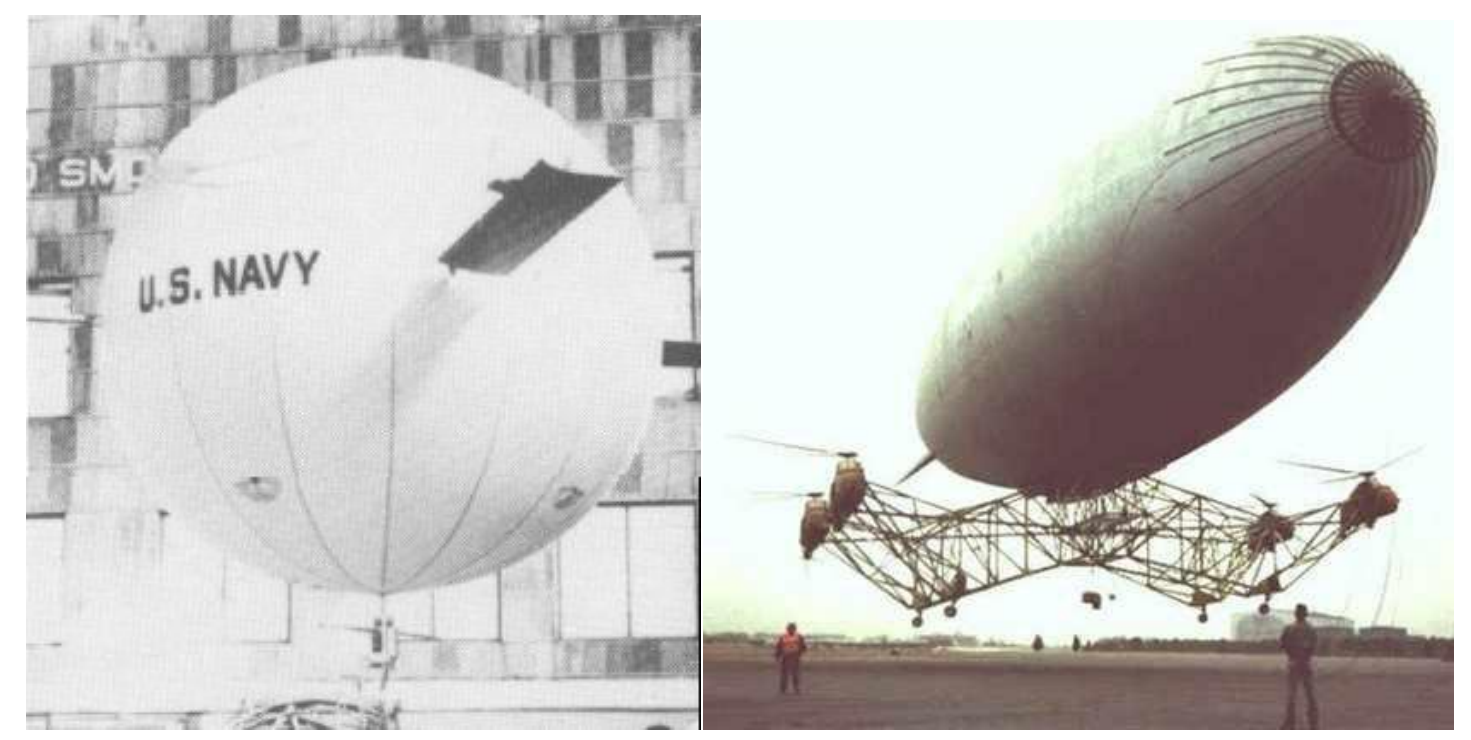

Figure 1-5: (Left) Aerocrane Scale Model Tested by the Navy and (Right) Heli-Stat Heavy-lift Vehicle [7]

As airships were seeing more and more attention, it was inevitable that the military would regain an interest. In 1996 a joint program between the Army, Navy, and Air Force began concept studies on a long-duration aerostat sensor platform. The intent was to provide "widearea surveillance and precision-tracking data to support a cruise missile defense system" [19]. The program developed slowly over the following years and was in turn abandoned. Eventually the program's original consideration would return with modified goals to support multiple highaltitude applications. This evolved into feasibility studies and concept evaluations for future 
airship projects. The military's revived attention meant once abandoned research and projects could serve as practical resources for these revised attempts.

In the past few years, less ambitious projects have successfully introduced modern LTAs into military service. Since 2004 the Army has operated tethered aerostats equipped with "multimission sensors to provide long endurance intelligence, surveillance, reconnaissance and communications" [20]. Lockheed Martin produces the unmanned aircrafts which offer "lowcost, continuous communications and persistent surveillance capabilities" [21]. Their implementation in Afghanistan has been very effective for enemy surveillance, and so far the Army has ordered 37 units. This demand has increased drastically over the past year according to the USA Today. The boom is a result of production time, cost, and effectiveness of the aerostats. They offer quicker production at about half the expense of the military's unmanned Predator drones. They are so effective at surveillance, using cameras that can see 10 to 15 miles away, that they help deter attacks according to Carter [22].

A greater increase in military service is expected for future airships, including larger scale surveillance duties, and heavy lifting platforms. Omari, et al. [23] have numerically evaluated a large-dimensioned airship for freight transport. Around 300 meters long, it is intended to carry up to 250 tons in an economic manner. The benefits of high altitude airships (HAA) have also received recent attention. A 2005 Rand Corporation report [17] examined the potential of such a craft for the Army. The military's desires are for an advanced surveillance and communication network for battle field support. HAA's have the potential to supplement the increasing demand for satellite communications with added benefits, including lower costs. Accordingly, specific performance goals were developed for such a project. They required an autonomous airship that was capable of continual geostationary operation for up to one year; all while maintaining an altitude of 70,000 feet. The HAA's potential was not lost on other military and civil applications. Its mobility benefits and mission-specific payload allow for uses spanning "short and long range missile warning, surveillance and target acquisition to communications and weather/environmental monitoring" [24]. Non-military disciplines have also taken notice, such as satellite communications. "Manufacturers are proposing high-altitude platforms to serve as surrogate satellites at a presumably reduced cost" [17].

The U.S. Military conducted feasibility studies that led to a contract with Lockheed Martin to develop and demonstrate a proof of concept model. An artist's rendering of their 
concept is pictured in Figure 1-6. It is fitted with photovoltaic cells and lightweight propulsion units to achieve the lengthy operation times. When performing duties at its maximum altitude of 70,000 feet, it would have a line-of-sight radial coverage of 325 miles. This potential range of coverage is superimposed over a map of Afghanistan below. Lockheed Martin is currently slated to demonstrate their development model with test flights in 2011 [21].

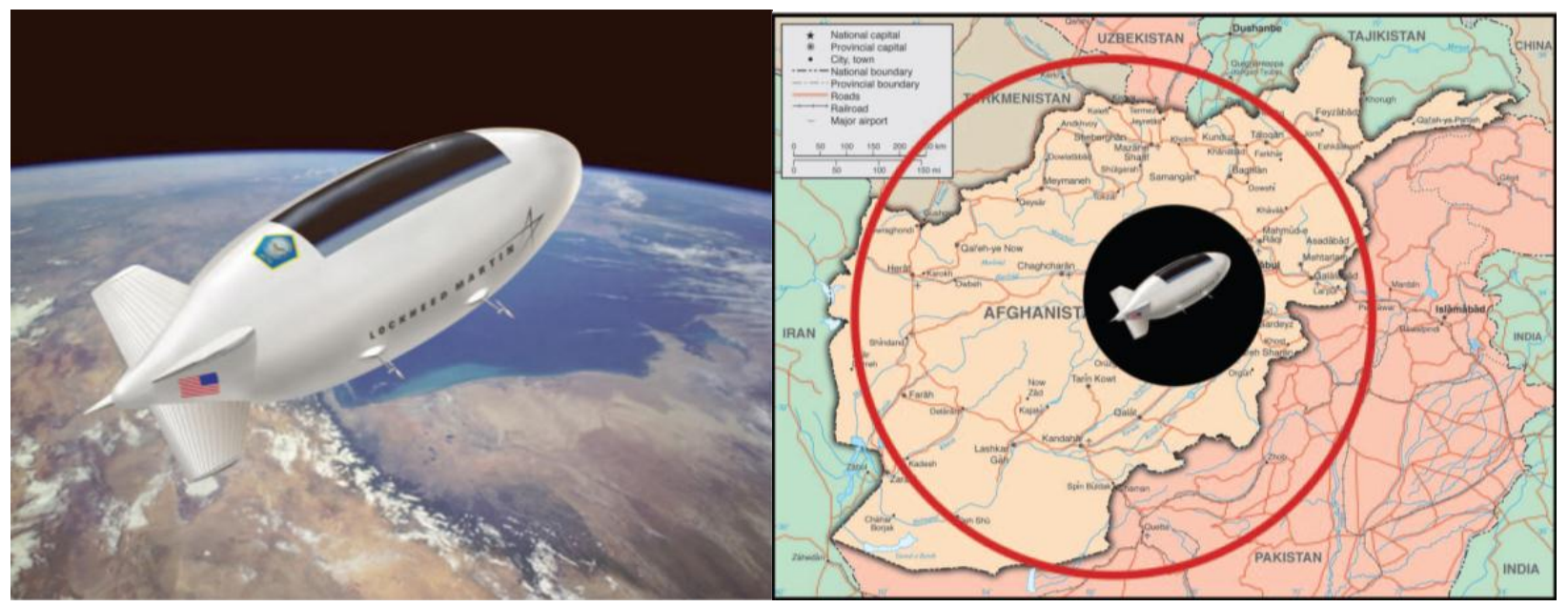

Figure 1-6: Artist Rendering of HAA Concept and Corresponding Line of Sight Capabilities at 70,000 feet [17]

Research into HAAs as well as hybrid and heavy lift airships continues to increase. Many designs, prototypes and fully operational models have already been evaluated. The Patuxent Partnership (TPP), which brings together industry, academia and government, hosts many conferences in support of airship projects. Topics for this year alone concerned hybrid airships, heavy lift applications, unmanned airships for battlefield communications, and futuristic applications in the space and defense industries. Their work is in support of the Department of Defense and Naval Aviation [25]. With a current and steady interest, airships are certain to fulfill an array of applications as they historically have. To meet today's desires necessitates a detailed range of analysis on the airship like never before.

CFD analysis has already been applied to several aspects of airship design. Kanikdale [26] has employed CFD to design an envelope for multi-disciplinary optimization. He examined aerodynamics for minimal volumetric drag and determined minimum surface area and hoop stress for structural considerations. Many studies have also examined experimental data for validating new methods or techniques. Researchers have considered non-linear dynamic terms like added-mass and resulting moments; some have developed new methods to calculate these 
more complicated terms [27] [28]. Thanks to today's technologies, CFD modeling can provide sophisticated analysis to modernize the airship for current and future applications. 


\section{Chapter 2 Relevant Airship Designs}

The apparent cyclical nature of airship usage continues, owing its present revival to the wide range of applications now being considered. This section discusses a prototype airship that began development during the Navy's PACE study. The prototype became the subject of numerous studies but never became fully operational. With present-day military considerations in mind the previous work serves as a logical starting point to modernize the airship for military use.

\subsection{Modern Military Needs}

The airship's return to significant use, at least for military applications, was anticipated after the Navy committed to an airship development program beginning in the 1980's. The airship's ability to carry large payloads, endure long flights, and provide a stable vibration free environment made it an ideal candidate for surveillance and communication roles. The Coast Guard joined with the Navy to develop a modernized airship by employing the latest available technologies. A major objective was to outfit the airship with modern avionics and computer technology and enhanced radar and detection systems, making it suitable for military surveillance. The Navy's specific interest was in developing an anti-ship missile defense system [19].

The proposed system would utilize a modern airship as an aerial platform that could house a radar system and other equipment to detect incoming missile threats. The Navyconfident that a modern airship could fulfill the role-awarded a \$170 million contract in 1987 to design and construct an operational development model that could demonstrate the airship's capability as an early warning system [18]. The contract afforded substantial research and development opportunities. This meant that the airship was to become an up-to-date aircraft for the first time in decades.

The Navy contract called for the development of a modern airship, designated YEZ-2A, from the ground up essentially beginning with design analysis and ultimately yielding a fully operational airship. The ambitions project began with determining the aircraft's design, i.e. size, fin configuration, thickness ratio etc. General features are shown in Figure 2-1. To develop any flightworthy aircraft requires an extensive assessment of performance and physical characteristics inherent in each design. Many differing setups and configurations were proposed 
and needed evaluation. Wind tunnel testing provided a custom environment for generating desired insight into most designs and configurations. Hence proposed designs were to be evaluated by testing scaled models.
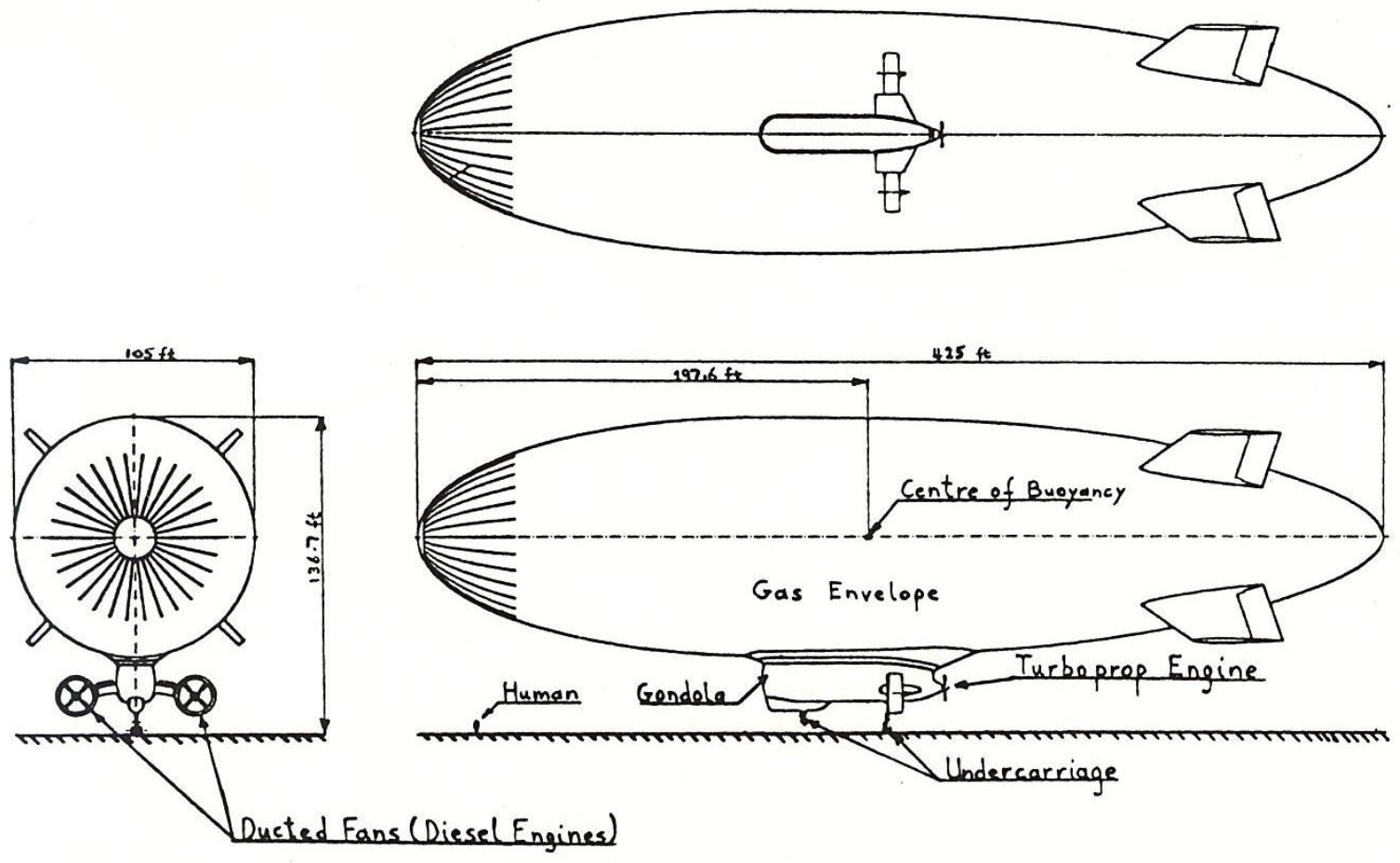

Figure 2-1: YEZ-2A General Features [4]

\subsection{YEZ-2A Airship}

\subsubsection{Wind Tunnel Testing}

Initial research on the YEZ-2A required an aerodynamic evaluation and the wind tunnel was to contribute the bulk of necessary data for the evaluation. The goal of typical wind tunnel testing is to reproduce full scale behavior associated with actual flight scenarios using a scaled model. It is often left to matching Reynolds numbers to assure these conditions are met. If this is not the case, as mentioned in Chapter 1, the most important parameter may be to reproduce transitions in the boundary layer at the correct relative location along the model. With this consideration and others, Gomes [4] performed scaled model testing and his work is described below.

For the YEZ-2A testing, a 1:75 scale size polyurethane model was constructed and shown for a test setup in Figure 2-3. This $1.72 \mathrm{~m}$ model required a tunnel velocity 75 times the full 
scale values to preserve Reynolds number matching. Achieving test velocities that high would introduce several complications and greatly limit the number of suitable facilities. Therefore the Reynolds number was not preserved. It is possible to achieve an effective Reynolds number by introducing certain techniques which alter the development of the boundary layer. Gomes accomplished this by incorporating a surface roughening strategy; effectively tripping the laminar boundary layer generating turbulent flow along the hull and avoiding flow separation. Consequently, the roughened surface affects the amount of skin friction drag but preserves form drag and lift. Since skin friction drag dominates airship hull drag, the resulting forces may yield an overestimation of drag. To alleviate this, a suitable level of surface roughness had to be determined based on flight test data [9].

Since the YEZ-2A existed only as a prototype, no flight test data was available to validate the modeling. However, a small amount of flight data for the Skyship-600 was available. This data was used with a 1:75 scaled model of its own to determine a suitable testing method, shown full scale in Figure 2-2 along with Gomes' model. Preliminary tests began with models featuring various degrees of surface roughness. These tests produced lift and drag data plots which were matched with flight test data to determine an appropriate level of roughness. This ensured that scale model testing could be performed at a $6 \times 10^{6}$ Reynolds number flow, around 35 times lower than full scale estimates. This flow was deemed appropriate considering the following: the rough surface effectively raised the test Reynolds number permitting insight into the full scale effects despite the large difference. It was also reasoned that the non-dimensional coefficients would vary only slightly with a Reynolds number increase above $6 \times 10^{6}$ since both regimes are characterized by fully turbulent flow [4]. 


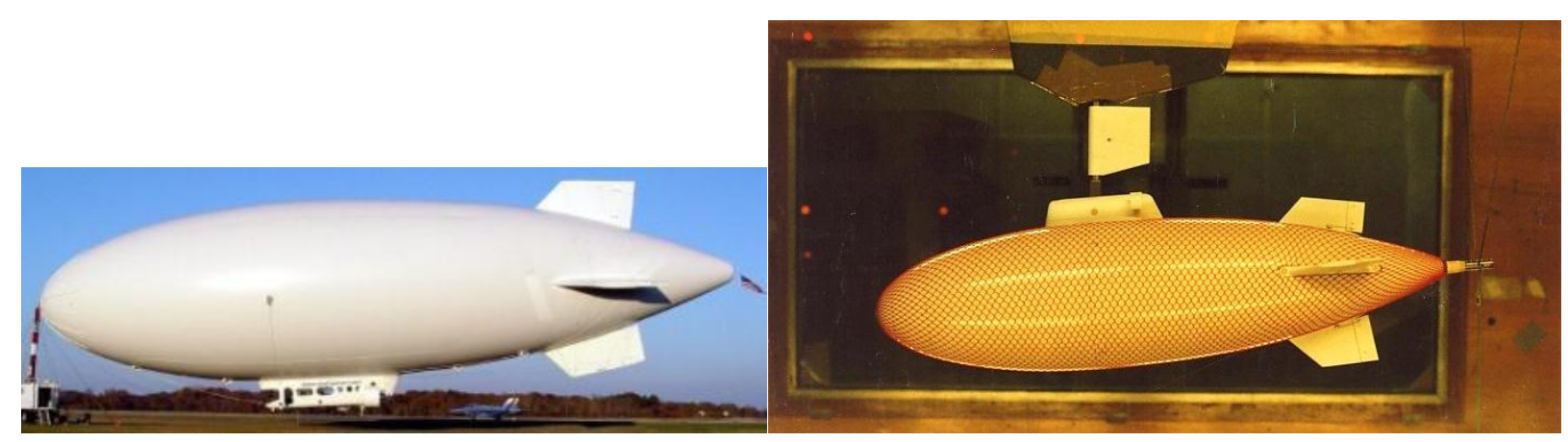

Figure 2-2: Skyship 600 Full Scale (Left) and 1:75 Model Scale (Right) [4]

The research resulted in a modeling approach that showed a good matching between scaled model data and available flight test values for the Skyship 600. The YEZ-2A was then modeled using this same method and level of surface roughness to yield the desired data during wind tunnel testing (Figure 2-3). Ultimately, this approach generated the experimental data required to evaluate the YEZ-2A's aerodynamics.

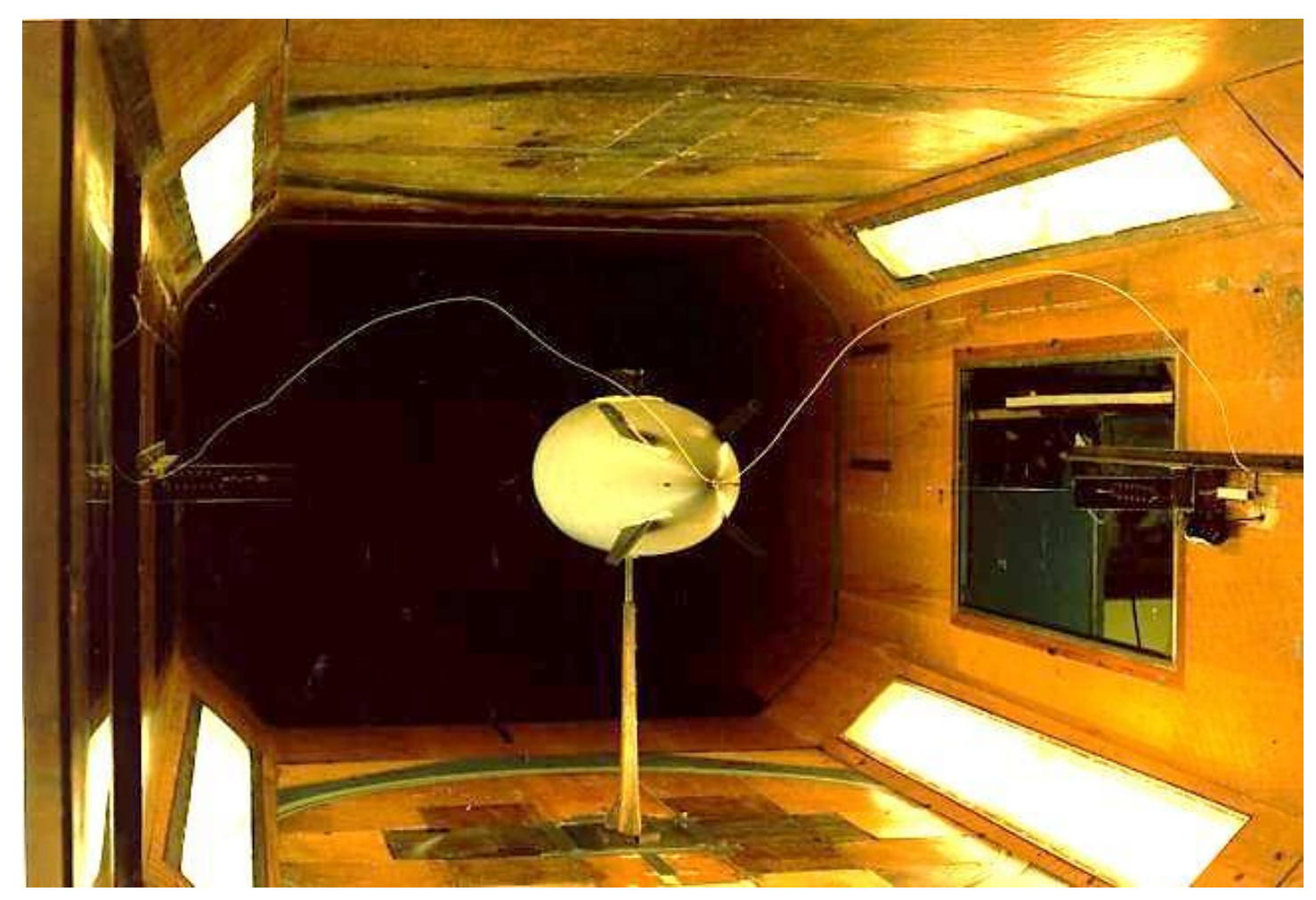

Figure 2-3: YEZ-2A 1:75 Scaled Model in Wind Tunnel Test Setup Used by Gomes [4]

Design analysis goals were achieved by Gomes' research [4], but the LTA program faltered and the project was canceled during construction of a full scale prototype in 1992 [19]. 
Military interest did not cease and eventually airship testing and research would return. Gomes' experiments became relevant once more but leaps in technological advancement presented new opportunities for analysis. The numerical capabilities of modern computers could provide parallel investigations with wind tunnel data, as well as more comprehensive evaluations. Therefore, CFD analysis ensued, providing an opportunity for this research.

\subsubsection{CFD Investigation}

With the renewed interest, West Virginia University (WVU) was contracted to perform an aerodynamic evaluation of the YEZ-2A airship using CFD analysis to determine whether numerical flow simulations could be used as a future design tool. This work simulated wind tunnel experiments performed by Gomes. The major task was to develop a robust CFD model that could capture flow physics and produce comparable aerodynamic data to the work of Gomes. Analysis considered two scaled model airship configurations, a hull only and a hull with control surfaces, at five different angles of attack. The effects of the hull and control surfaces on lift, drag, and pitching moment were studied.

The modeling process began with an examination of meshing requirements, viscous model parameters, and solver requirements. The computational domain consisted of a spherical mesh surrounding the model that featured a finely structured near field grid that transitioned to a courser unstructured far field grid. A sensitivity study was used to select an appropriate turbulence model. The modeling process placed great emphasis on minimizing computational expenses while obtaining accurate analysis. This investigation introduced a successful modeling approach that generated acceptable aerodynamic data. Overall results compared favorably to the wind tunnel work of Gomes [5]. This constituted the preliminary WVU CFD work on the airship

\subsection{Objectives}

Current research continues the investigation into airship aerodynamics and was undertaken to expand upon previous CFD analysis. This investigation was concerned with analysis of the bare hull aerodynamics of an airship. Specifically the YEZ-2A airship was modeled and analyzed numerically for the 1:75 scale and full size configurations. Initial analysis involved reapplying techniques and procedures developed for the prior WVU CFD study on the 
scaled airship hull. This provided validation of prior work as well as insight and experience into the modeling procedures.

Grid and temporal independence of results were then considered by evaluating several axisymmetric models of the scaled hull and then again for the full scale hull. This analysis determined the appropriate level of grid refinement and an acceptable discretization scheme (transient or steady state) to use. These efforts were undertaken to reevaluate previous modeling and confirm the independence of results.

The final analysis was concerned with evaluating a series of full scale three dimensional models of the airship hull. Previous modeling procedures were employed once more to develop a high fidelity spherical domain. This time model attributes were matched to flight conditions, including a Reynolds number two orders of magnitude higher. The full sized model was evaluated to reveal potential errors caused by scaling effects, to provide additional conformation for previous results, and to further demonstrate the capability of CFD as a possible design tool.

Ultimately, this research was undertaken with a number of objectives present. The objectives include the demonstration of CFD as an airship design tool, evaluation of Gomes wind tunnel techniques and assumptions, and validation of previous investigations with CFD results. Great care was given to ensure the CFD analysis would produce realistic results independent of modeling factors. Data was validated wherever possible, using available results from prior studies. Difficulties associated with non-dimensional scaled modeling are examined as well as effects encountered during CFD analysis. An attempt was made to understand the physical processes resolved in the flow for each scenario. Modeling factors and boundary conditions were examined to reveal their individual contributions to the physical flow structure, as they ultimately govern aerodynamic forces. This research can be considered an extension of the previous YEZ-2A investigations as well as a preliminary guide to implementation of CFD analysis as a practical tool for airship design. 


\section{Chapter 3 Numerical Approach/Grid and Model Setup}

This chapter discusses research performed herein, examining CFD development from grid generation up to solution initialization. Research included the use of Gambit, a model generator, along with Tgrid and Fluent, a grid generator and analytical software, respectively [29]. Preprocessing requirements included the generation of two distinct geometrical setups (axisymmetric and 3D) for each model type (1:75 scale and full scale). Axisymmetric models were created for three levels of grid refinement for each model and three dimensional models were created for three different angles of attack (AoA) for each model. Relevant data was collected from a total of 12 models, half corresponding to three dimensional setup and half as axisymmetric, as well as half full sized and half 1:75 scale. Table 3-1 shows general features of the evaluated models. These models were selected after examining a variety of grids and designs until an appropriate grid geometry ( $\mathrm{y}+$ values and aspect ratios) could be achieved.

Table 3-1: General Features of CFD Models

\begin{tabular}{|ccccc|}
\hline Model & Type & Scale & Grid Resolution & AoA \\
\hline 1 & axisymmetric & $1: 75$ & course & $0^{\circ}$ \\
2 & axisymmetric & $1: 75$ & medium & $0^{\circ}$ \\
3 & axisymmetric & $1: 75$ & fine & $0^{\circ}$ \\
4 & axisymmetric & Full & course & $0^{\circ}$ \\
5 & axisymmetric & Full & medium & $0^{\circ}$ \\
6 & axisymmetric & Full & fine & $0^{\circ}$ \\
\hline 7 & 3D & $1: 75$ & medium & $0^{\circ}$ \\
\hline 8 & 3D & $1: 75$ & medium & $5^{\circ}$ \\
9 & 3D & $1: 75$ & medium & $10^{\circ}$ \\
10 & 3D & Full & medium & $0^{\circ}$ \\
11 & 3D & Full & medium & $5^{\circ}$ \\
12 & 3D & Full & medium & $10^{\circ}$ \\
\hline
\end{tabular}

\subsection{Grid Construction}

\subsubsection{D Mesh Setup}

CFD analysis began with the grid generation process with careful consideration given to setup and design. The modeling approach used in previous WVU studies [5] was employed here for generating three dimensional models. The process began by introducing a sphere around the 3D airship hull and meshing each surface. The airship hull received a structured quad mapped mesh (Figure 3-1) where the longitudinal cell count was matched to that of the previous WVU 
study and a corresponding lateral cell count was selected for minimal skewness and aspect ratio. The outer sphere, representing the far field, then received an unstructured surface mesh generated with a sizing function (Figure 3-2). Far field dimensions extended out radially 10 major hull diameters. This was a suitable dimension recommended for external flow problems [30]. A structured near field grid surrounding the hull was then generated by growing prism layers out from the meshed hull surface. Creating the far field mesh involved growing an unstructured mesh from the near field surface to the far field. The spherical design permitted direct application of the free stream flow which eliminated the need to generate entirely new models for each angle of attack. Altering the angle from model to model involved reorienting the hull with respect to the spherical far field before applying the above construction procedures, allowing for a simplified and consistent modeling process overall. 


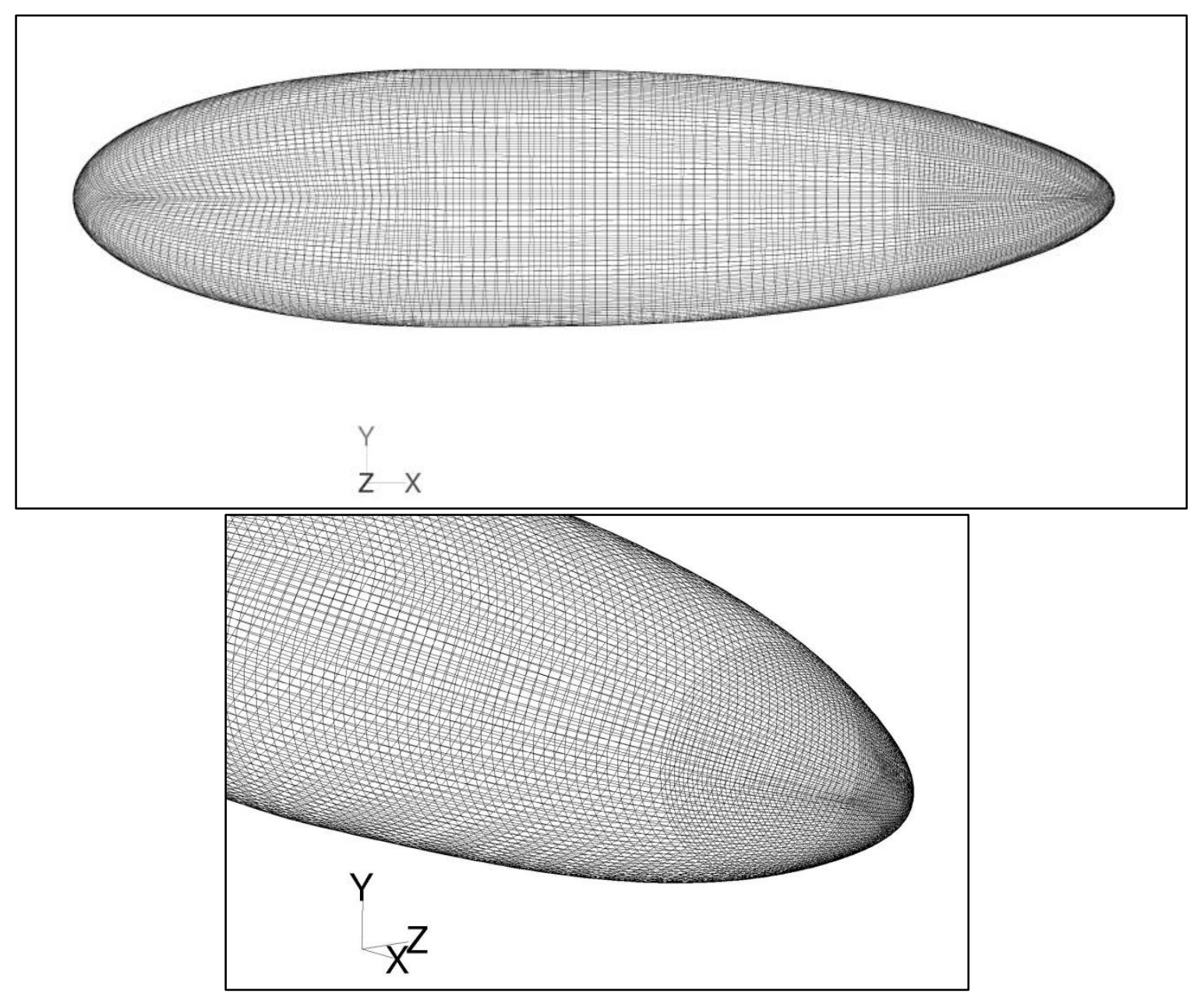

Figure 3-1: Structured Hull Surface Mesh of 1:75 Scale YEZ-2A Airship Hull: (Top) Longitudinal View and (Bottom) Angled View of Tail Mesh 

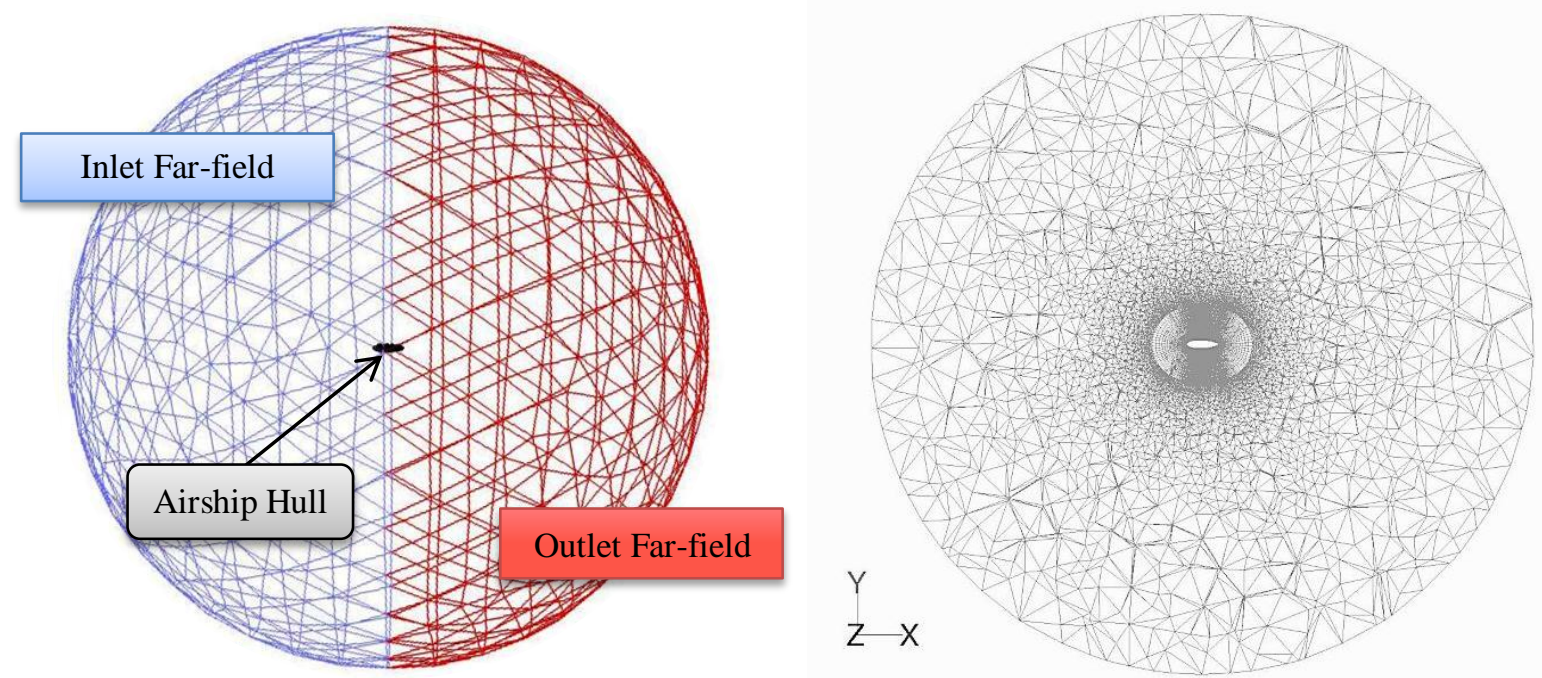

Figure 3-2: (Left) Unstructured Far-field Mesh Surrounding Airship Hull with Internal Mesh Removed for Clarity and (Right) Horizontal Slice Showing Interior View of Total Mesh

This modeling approach allowed for a high fidelity structured near-field mesh, with a transition to the unstructured far field where cell size was expanded outward from the hull surface by introducing a growth rate into the grid development. This process allowed for cell volume expansion, which greatly reduced computational expenses, and maintained consistent mesh attributes between models. Cell expansion can be seen in Figure 3-3 as well as the structured to unstructured transition. This method was developed in part for the previous WVU study and employed during the current research for its sound numerical approach and to minimize modeling discrepancies between studies. 


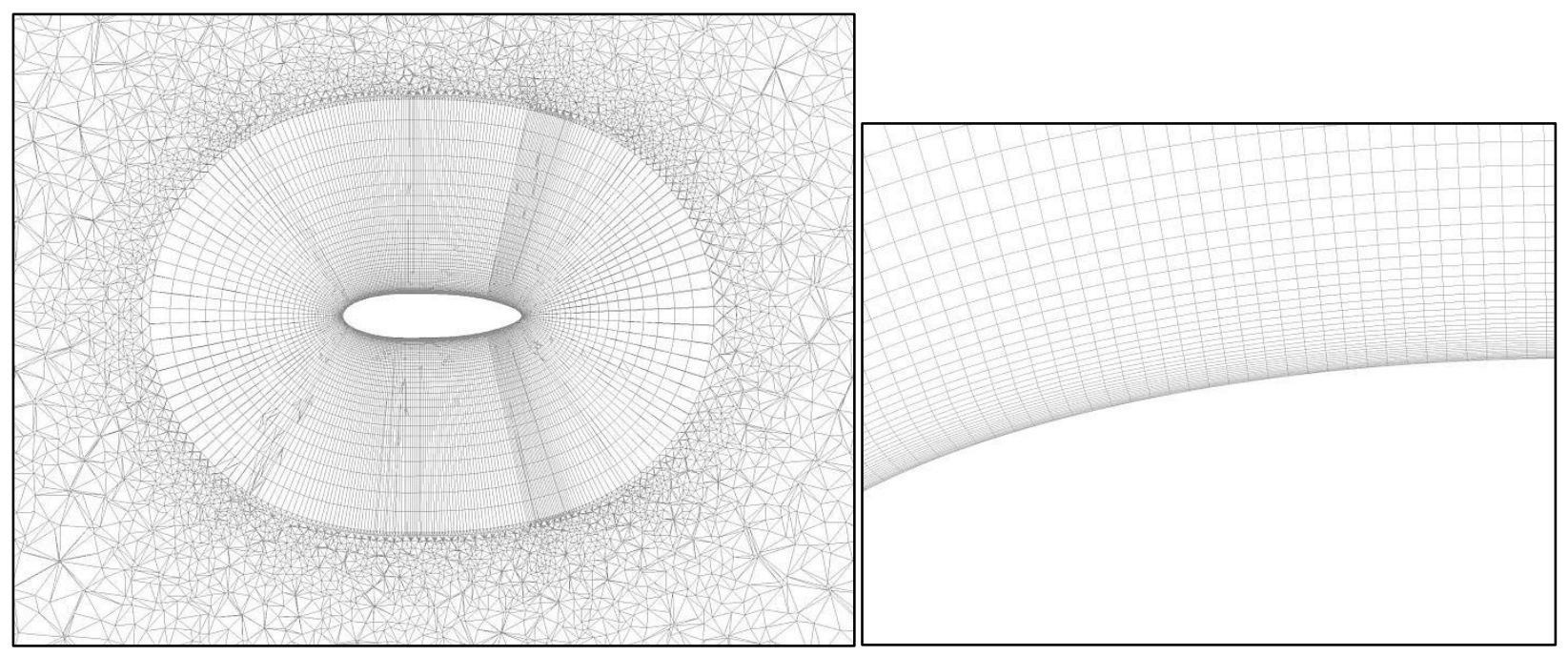

Figure 3-3: (Left) Complete Near-field View and Near-wall Zoom View (Right) of the 3D Airship Mesh

\subsubsection{Axisymmetric Mesh Setup}

A separate grid construction effort was undertaken to develop a series of axisymmetric models. These models were developed for subsequent grid and temporal independence studies concerning model scale and full scale sizes of the YEZ-2A airship hull. The grid independence study required three geometrically similar grids with an increase in grid refinement from one to the next. Therefore, course, medium, and fine grids were constructed for both hull sizes yielding a total of six axisymmetric grids. Using Gambit, a two dimensional hull profile was first split longitudinally and then encompassed with a hemispherical far field as seen in Figure 3-4. The axisymmetric models were all meshed using a structured quad mapped mesh. Grid resolution was varied according to the interval count along the hull as shown in Figure 3-5. The first cell height at the wall was selected in accordance with wall function $y+$ values to ensure critical flow physics were captured while minimizing computational expenses. Recommended upper and lower bounds for $\mathrm{y}+$ were 300 and 30, respectively; a value close to the lower bound was ideal [29]. Values of $\mathrm{y}+$ along the hull are shown in Appendix $\mathrm{C}$ and are within suggested limits. Finally, a sizing function was introduced that allowed cells to expand as they progressed away from the hull, further reducing expenses. Features of the resulting scaled model grids are shown in Table 3-1. 


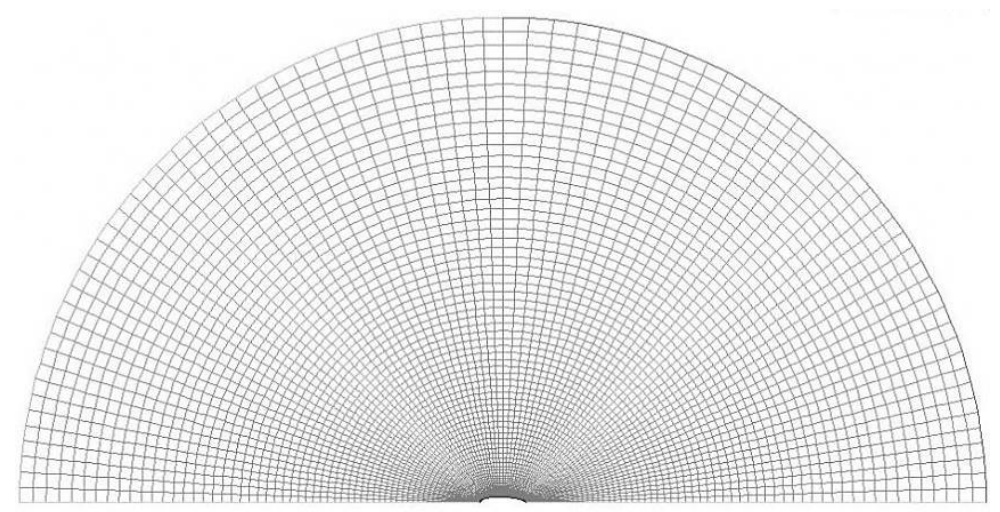

Figure 3-4: Complete Hemisphere Grid Surrounding Airship Hull for Axisymmetric Models

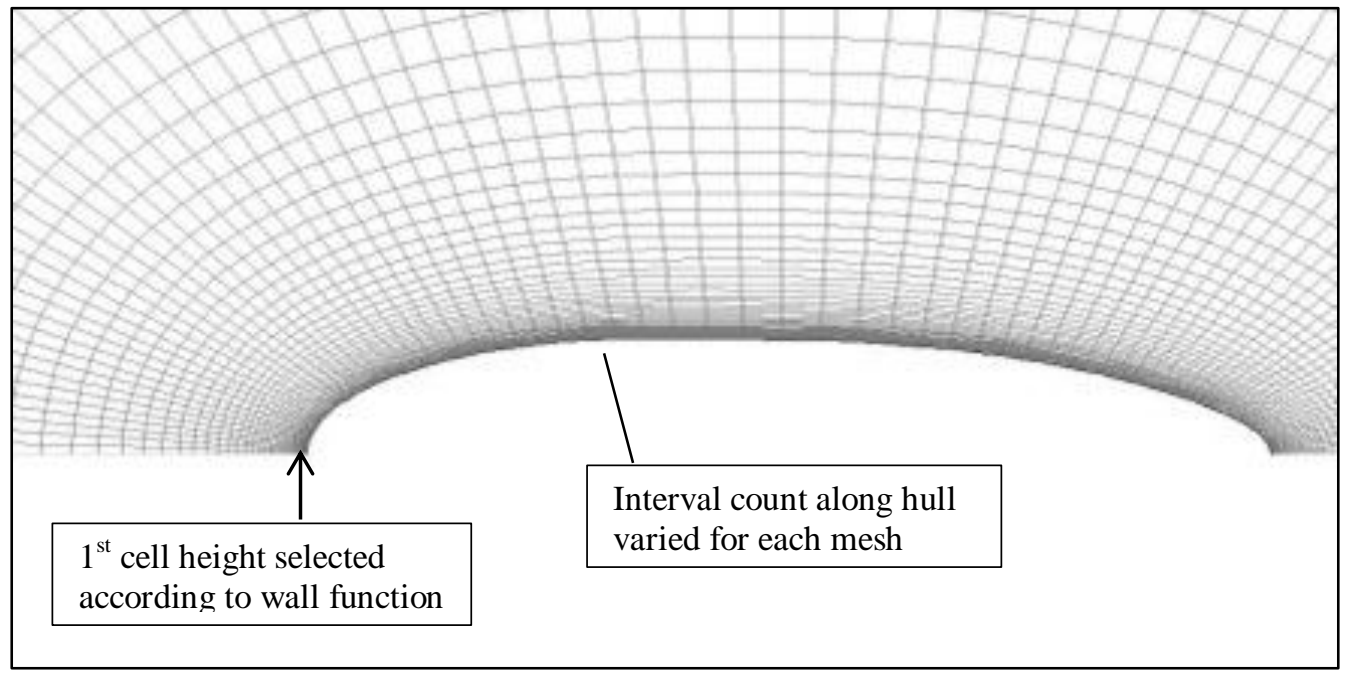

Figure 3-5: Zoomed Hull Region Showing Near-wall Grid of Course Mesh

Table 3-2: 1:75 Scale Axisymmetric Mesh Densities

\begin{tabular}{|cccc|}
\hline Model & $\begin{array}{c}\text { Grid } \\
\text { Resolution }\end{array}$ & $\begin{array}{c}\text { Hull Interval } \\
\text { Count }\end{array}$ & Cells \\
\hline 1 & course & 80 & 8000 \\
2 & medium & 120 & 12000 \\
3 & fine & 180 & 18000 \\
\hline
\end{tabular}

The generation of full scale axisymmetric grids with comparable resolution would involve a significant increase in computational expenses. To reduce this, a 5:1 boundary layer was introduced into the grid as shown in Figure 3-6. The boundary layer was positioned beyond the finely structured near field as a geometric tool to reduce cell interval count by $80 \%$ as it expands into the far field. The boundary layer allowed for a fine structured mesh capable of capturing near field physics to transition to a less resolved mesh suitable for capturing far field 
flow. Full scale models were generated with grids featuring resolutions shown in Table 3-3. With the exception of the boundary layer region and dimensions, grid design was identical between scaled and full sized models.

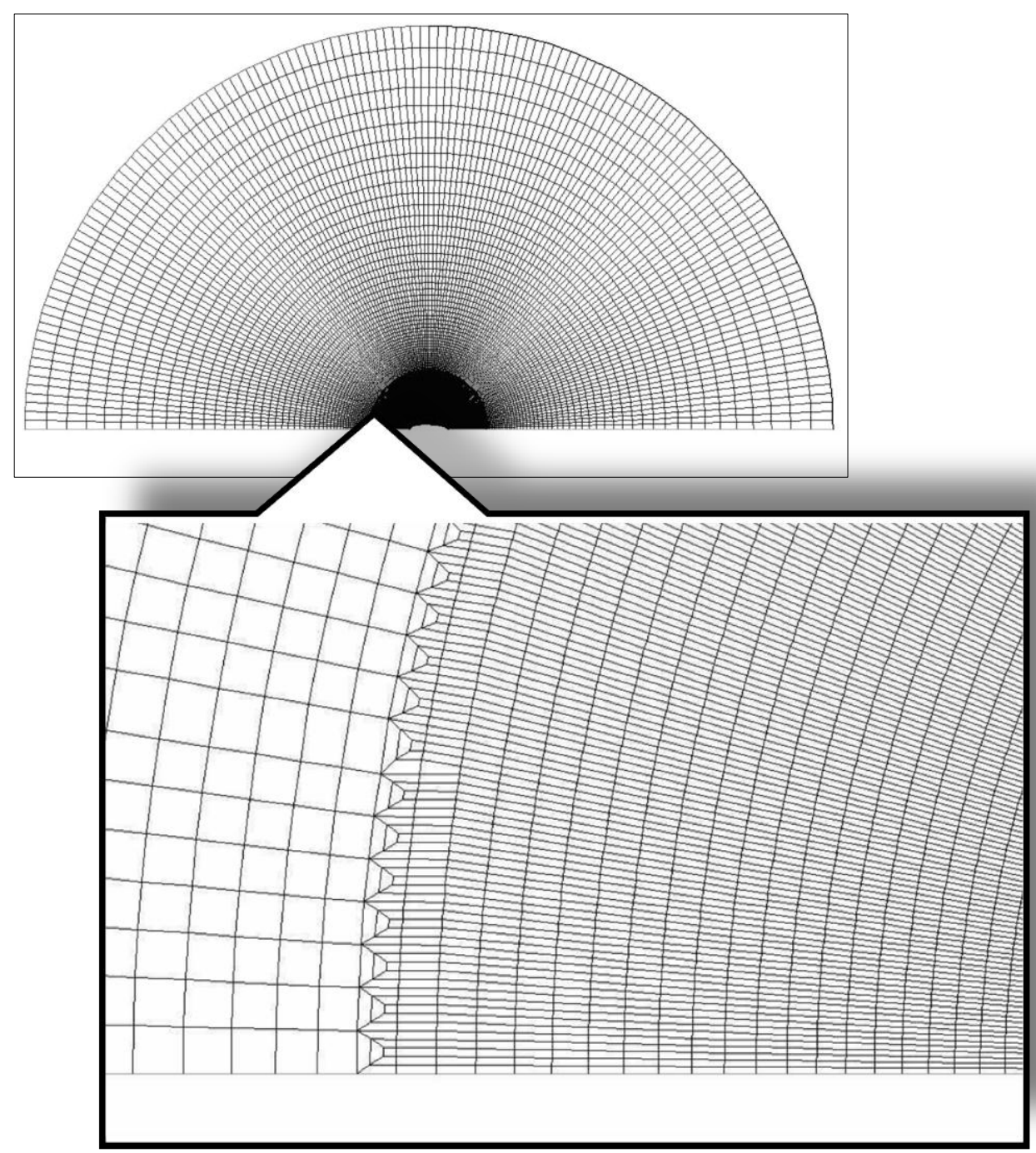

Figure 3-6: Axisymmetric Full Scale Grid with Zoomed Boundary Layer Region

Table 3-3: Full Scale Axisymmetric Mesh Densities

\begin{tabular}{|cccc|}
\hline Model & $\begin{array}{c}\text { Grid } \\
\text { Resolution }\end{array}$ & $\begin{array}{c}\text { Hull Interval } \\
\text { Count }\end{array}$ & Cells \\
\hline 1 & course & 700 & 98700 \\
2 & medium & 1000 & 141000 \\
3 & fine & 1400 & 197400 \\
\hline
\end{tabular}




\subsection{Modeling and Boundary Conditions}

Following grid construction was model setup and application of boundary conditions. Modeled flow was dominated by turbulence with an order of $10^{6}$ Reynolds number. Since there exists a variety of turbulence models in Fluent, the model selection process was based on a sensitivity study. It was shown in previous work [5] that the two equation SST k- $\omega$ turbulent viscosity model was the most suitable model for the given geometry and flow conditions. This selection was based on its computational efficiency as well as its accuracy in calculating external aerodynamic flow problems, as determined by the sensitivity study.

As described in Chapter 1, modeling of turbulence in CFD is a challenging process. This modeling is challenged further when additional complexities are introduced; separated flows, three dimensionality, and irregular geometries are a few examples. Many modeling techniques exist to analyze these complicated flows. The effectiveness of these models for a variety of flows has been examined frequently and has been the subject of many conferences. Studies revealed that no single method was universally successful across the range of possible cases. Likewise no method proved entirely ineffective, as each method found success for certain cases [11].

Omari, et al. examined the capabilities of three turbulence models when applied to an airship at a high AoA. They examined a standard high Reynolds $\mathrm{k}-\varepsilon$ model $-\mathrm{a}$ two equation statistical Reynolds-averaged-Navier-Stokes (RANS) model, a Smagorinsky Large Eddie Simulation (LES) model, and a variational multiscale LES (VMS-LES) one. Simulations were compared to experimental observations to gage each model's performance. Results indicated that the RANS and LES models introduced too much turbulent viscosity which damped turbulent structures. Unlike these the VMS-LES model captured a secondary vortex that agrees with experiments and numerical studies at higher Reynolds numbers. The models were equally capable of predicting surface pressures and required comparable CPU resources. Overall the LES based models are restricted to lower Re number flows where the RANS is not [23].

The sensitivity study performed by Menchini, et al. considered several RANS models due to the high level of turbulence present $\left(6 \times 10^{6}\right)$. Fluent provides several one and two-equation models that provide a steady mean flow field where small-scale turbulent fluctuations are modeled. Additional terms are added to the classical set of conservation equations, increasing 
the number of unknowns present, which are modeled to provide solution closure. The following excerpt is taken from Menchini, et al. [5]:

An entire family of one and two-equation turbulence models exist tailored for a variety of general turbulent flow applications, along with specialized derivatives to capture critical effects. Statistics of a time dependent flow field such as time averaged mean and root mean square velocities can be solved using the RANS approach, which are typically of the most interest to engineering applications. Some of these models, such as the Spallart-Almaras, $\mathrm{k}-\varepsilon$, and $\mathrm{k}-\omega$, assume the Boussinesq hypothesis, whereby the Reynolds stresses imposed by RANS are related by mean velocity gradients. Although applicable for certain flow regimes, this approach assumes turbulent viscosity is isotropic, which in general, for real world applications is not completely accurate. The majority of these models track a turbulent kinetic energy in addition to a turbulent dissipation rate equation to account for the presence and interaction of fluctuating turbulence quantities with the mean flow. Reynolds Stress Modeling (RSM) solves the entire Reynolds Stress tensor without using the Boussinesq hypothesis, although more computationally expensive, taking into account anisotropic affects that may arise in a turbulent flow field.

All flow physics outlined represent the solution of six conservation equations (continuity, three-dimensional momentum, in addition to a two equation turbulence model) except for the Spallart-Almaras model which is a one equation eddy-viscosity turbulence model, along with the Reynolds Stress Model (RSM) which adds an additional six conservation equations beyond the two equation models mentioned earlier (three removed due to tensor symmetry). Due to enhanced memory management techniques and the unique mathematical coupling present within the Reynolds stress tensor, iterations are optimized within the ANSYS Fluent framework resulting in a less than expected proportionate memory and CPU clock requirement increase.

Results of the sensitivity study are shown in Table 3-4. Presented is a comparison of evaluated turbulence models and their approximate computational requirements. Each model was evaluated for the $20^{\circ}$ AoA case since turbulence effects are greatest at this angle. Several parameters were varied among the cases, including solver selection and turbulent boundary conditions. Initially, turbulence models were evaluated using a steady flow solver. Results were compared with wind tunnel data and the SST k- $\omega$ was selected based on its computational efficiency and its accuracy in calculating external aerodynamic flow problems. Subsequent cases investigate the selected model under transient conditions for three different time steps. Resulting flow structures were qualitatively identical between flow solvers and coefficients varied by no more than $3 \%$, indicating the steady flow solver was reasonable for analysis. Variation of turbulent inlet conditions showed a negligible effect on the resulting flow. This may be due to a high level of turbulence already present at the flow boundary, making calculations 
insensitive to inflow boundary conditions [29]. Accordingly, turbulent intensity was selected and its value was determined by matching wind tunnel data of Gomes. Length scale was based on an empirical approach for external aerodynamic flows. Its value equaled 0.07 times a characteristic length of the body, in this case the minor diameter of the airship.

Table 3-4: CFD Model Sensitivity Analysis Using the 1:75 Scale Hull with Fins Configuration Adapted from Menchini, et al. [5]

\begin{tabular}{|c|c|c|c|c|c|c|}
\hline $\begin{array}{l}\text { Case } \\
\text { No. }\end{array}$ & Flow Physics & $\begin{array}{c}\text { Wall } \\
\text { Treatment }\end{array}$ & $\begin{array}{c}\text { Grid } \\
\text { Topology }\end{array}$ & $\begin{array}{l}\text { Node } \\
\text { Count }\end{array}$ & $\begin{array}{l}\text { CPU Count/Clock } \\
\text { Speed/Memory }\end{array}$ & $\begin{array}{l}\text { Solution Time } \\
\text { (Total CPU Hours) }\end{array}$ \\
\hline 7 & $20^{\circ}$ AoA SST k- $\omega$ Turbulence TI\% - 0.1/LS - $0.02975 \mathrm{~m}$ & Wall Function & $3 \mathrm{D}$ & $1 \mathrm{M}$ & $2 / 2.5 \mathrm{Ghz} / 4 \mathrm{~GB}$ & 16 \\
\hline 8 & $20^{\circ}$ AoA SST k- $\omega$ Turbulence TI\% - 1/LS - $2.975 \mathrm{~m}$ & Wall Function & $3 D$ & $1 \mathrm{M}$ & $2 / 2.5 \mathrm{Ghz} / 4 \mathrm{~GB}$ & 16 \\
\hline 9 & $20^{\circ}$ AoA SST k- $\omega$ Turbulence TI\% - 10/LS - $29.75 \mathrm{~m}$ & Wall Function & $3 \mathrm{D}$ & $1 \mathrm{M}$ & $2 / 2.5 \mathrm{Ghz} / 4 \mathrm{~GB}$ & 16 \\
\hline 10 & $20^{\circ}$ AoA SST k- $\omega$ Turbulence TI\% - 10/VR - 5 & Wall Function & 3D & $1 \mathrm{M}$ & $2 / 2.5 \mathrm{Ghz} / 4 \mathrm{~GB}$ & 16 \\
\hline 11 & $20^{\circ}$ AoA SST k- $\omega$ Turbulence TI\% - 10/VR - 10 & Wall Function & $3 \mathrm{D}$ & $1 \mathrm{M}$ & $2 / 2.5 \mathrm{Ghz} / 4 \mathrm{~GB}$ & 16 \\
\hline 12 & $20^{\circ}$ AoA Real k- $\varepsilon$ Turbulence TI\% - 0.1/LS - 0.02975 m & Wall Function & $3 D$ & $1 \mathrm{M}$ & $2 / 2.5 \mathrm{Ghz} / 4 \mathrm{~GB}$ & 16 \\
\hline 13 & $20^{\circ}$ AoA S-A Turbulence TI\% - 0.1/LS - $0.02975 \mathrm{~m}$ & Wall Function & $3 D$ & $1 \mathrm{M}$ & $2 / 2.5 \mathrm{Ghz} / 4 \mathrm{~GB}$ & 16 \\
\hline 14 & $20^{\circ}$ AoA RSM Turbulence TI\% - 0.1/LS - $0.02975 \mathrm{~m}$ & Wall Function & 3D & $1 \mathrm{M}$ & $2 / 2.5 \mathrm{Ghz} / 4 \mathrm{~GB}$ & 16 \\
\hline 15 & $\begin{array}{l}20^{\circ} \text { AoA SST k- } \omega \text { Turbulence TI\% - 0.1/LS }-0.02975 \mathrm{~m} \\
\text { Unsteady } \Delta \mathrm{t}=1 \mathrm{e}-4 \mathrm{~s}\end{array}$ & Wall Function & $3 \mathrm{D}$ & $1 \mathrm{M}$ & $2 / 2.5 \mathrm{Ghz} / 4 \mathrm{~GB}$ & 16 \\
\hline 16 & $\begin{array}{l}20^{\circ} \text { AoA SST k- } \omega \text { Turbulence TI\% }-0.1 / L S-0.02975 \mathrm{~m} \\
\text { Unsteady } \Delta \mathrm{t}=0.01 \mathrm{~s}\end{array}$ & Wall Function & $3 D$ & $1 \mathrm{M}$ & $2 / 2.5 \mathrm{Ghz} / 4 \mathrm{~GB}$ & 16 \\
\hline 17 & $\begin{array}{l}20^{\circ} \text { AoA SST k- } \omega \text { Turbulence TI\% - 0.1/LS }-0.02975 \mathrm{~m} \\
\text { Unsteady } \Delta \mathrm{t}=0.1 \mathrm{~s}\end{array}$ & Wall Function & $3 D$ & $1 \mathrm{M}$ & $2 / 2.5 \mathrm{Ghz} / 4 \mathrm{~GB}$ & 16 \\
\hline
\end{tabular}

Real k- $\varepsilon$ : Realizable k- $\varepsilon$ Turbulence Model

S-A: Spallart-Almaras Turbulence Model

SST k- $\omega$ : SST k- $\omega$ Turbulence Model

RSM: Reynolds Stress Turbulence Model

TI\%: Turbulence Intensity = (VRMS/Vmean)

LS: Characteristic Turbulent Length Scale

VR: Ratio of Turbulent to Molecular Viscosity $(\mu \mathrm{T} / \mu \mathrm{L})$

Turbulent Boundary Condition Specifications (Either defined TI + LS or TI + VR)

Completing the model setup, boundary conditions were applied, aided by the domain's central division effectively separating inlet and outlet flow conditions (Figure 3-7). This setup was used for both small scale and full scale three dimensional models, with the critical flow parameters for each shown in Table 3-5. Ultimately the model design and setup matched that of the previous WVU study. The aforementioned considerations provide a consistent modeling approach to minimize discrepancies between studies, subsequently reducing uncertainty in result comparisons. 


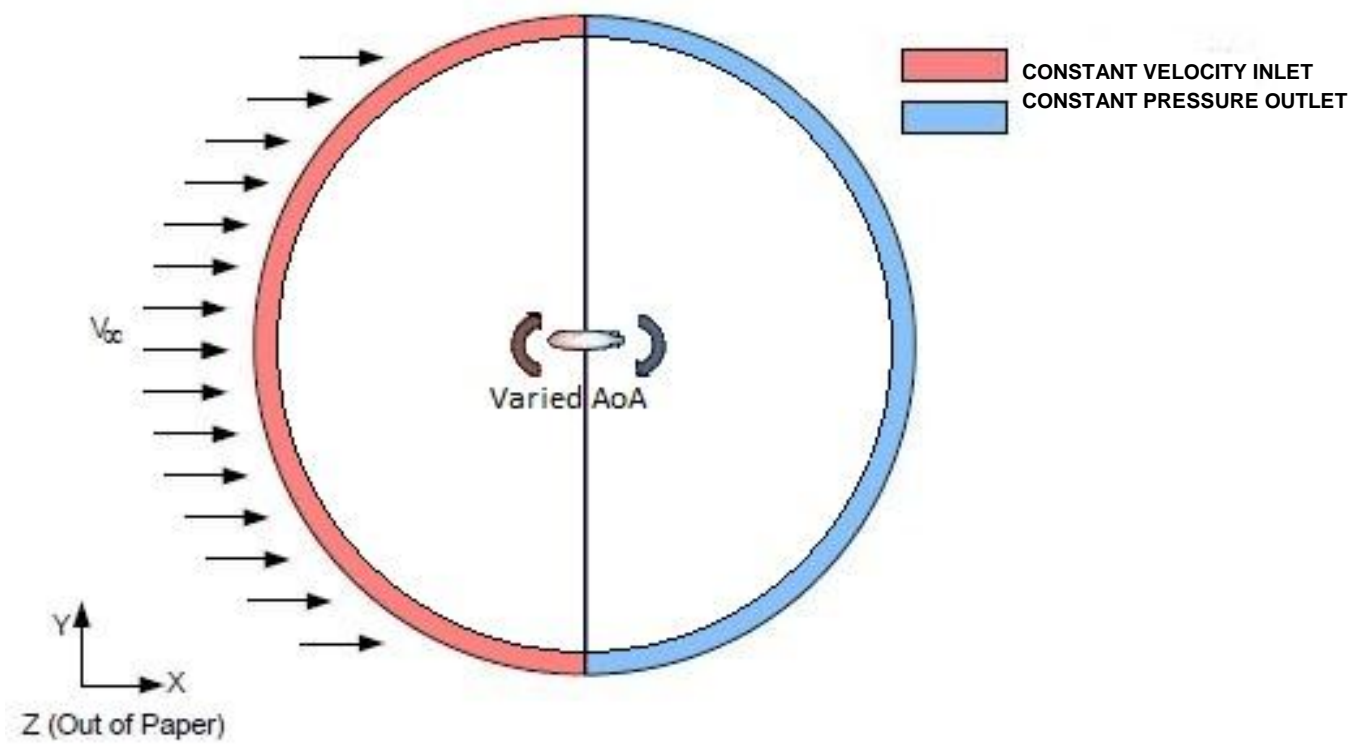

Figure 3-7: 3D Model Overview with Boundary Conditions

Table 3-5: Hull Dimensions and Critical Flow Parameters Applied to CFD Models

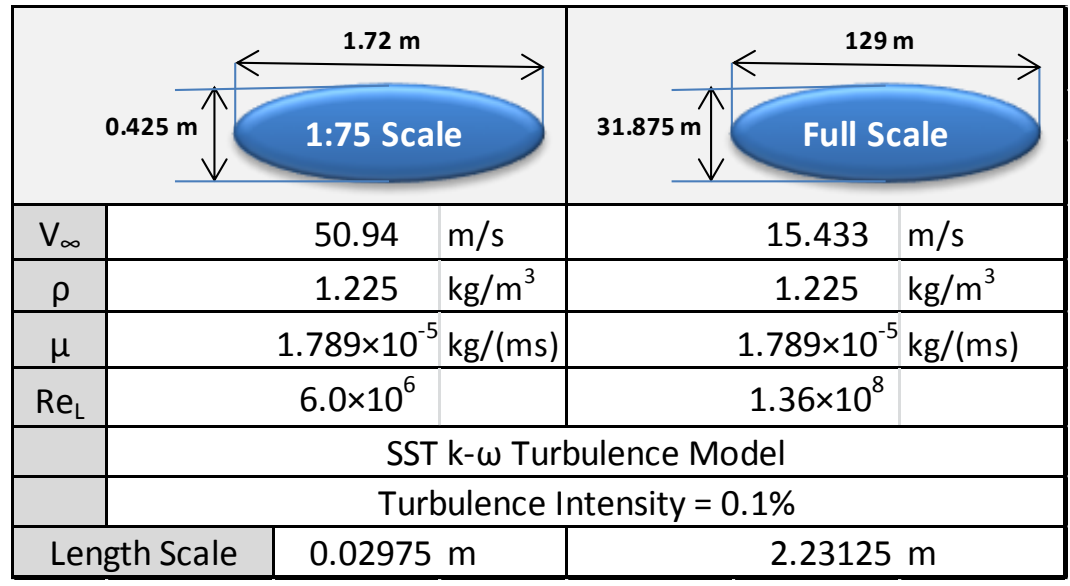

Figure 3-8 shows the setup applied to axisymmetric models. Turbulence model selection and flow parameters were matched to the three dimensional study, displayed in Table 3-5. Boundary conditions were applied in a similar fashion with the use of a wall function. These parameters were preserved not only for their modeling benefits but also to permit direct comparisons among resulting data. 


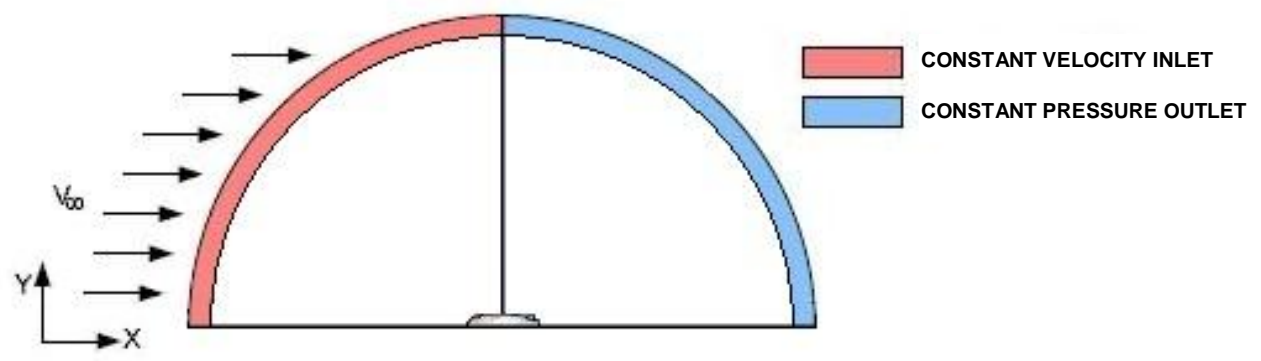

Figure 3-8: Axisymmetric Model Overview with Boundary Conditions 


\section{Chapter 4 CFD Analysis, Results and Discussion}

This research employed CFD analysis to examine full scale models and 1:75 scale models of the YEZ-2A airship hull. The previous investigations provide data to validate the resulting CFD approach as well as an opportunity to assess techniques and assumptions encountered previously. This section presents results of the research and provides a comparative analysis to the previous investigations.

The generation of small and full scale three dimensional models was influenced heavily by the results of previous investigations and sensitivity studies. Constraints on grid geometries resulted from a grid refinement study, solver setup was dictated by the transient analysis study, and turbulence model selection as well as model design was influenced by the previous WVU study.

The three dimensional model grids were designed to be geometrically similar to those selected in the axisymmetric study by considering aspect ratio, cell interval count, skewness, cell height, and mesh mapping. Model design and boundary conditions-featuring the airship hull encompassed by a spherical domain, split equally for the inlet and outlet zones - allude to the setup used in the previous WVU study [5].

\subsection{Numerical Data}

\subsubsection{D 1:75 Scale Models}

The three dimensional modeling procedures, discussed in Chapter 3, were applied to the hull-only scaled model setup for three different angles of attack $\left(0^{\circ}, 5^{\circ}\right.$ and $\left.10^{\circ}\right)$. This was followed by executing 1000 first order, implicit, steady state iterations. These iterations were used for solution initialization of subsequent second order computations. Analysis was completed by evaluating the second order, implicit, steady state iterations until convergence was achieved.

The resulting aerodynamic coefficients are shown in Table 4-1. These data show good agreement with those determined in the previous WVU study. Additional data are displayed in Appendix A, including contour plots of velocity magnitude, pressure coefficient and shear stress for each AoA setup. Contour plots such as Figure 4-1 adequately represent expected flow physics, thereby suggesting the model and setup are appropriate selections for this application. 
Overall the data conform to what was expected showing an increase in lift and drag with an increase in angle of attack.

Table 4-1: 1:75 Scaled Model Lift and Drag Coefficients

\begin{tabular}{|c|c|c|}
\hline $\begin{array}{c}\text { AoA } \\
\text { (degree) }\end{array}$ & $\mathbf{C}_{\mathrm{L}}$ & $\mathbf{C}_{\mathrm{D}}$ \\
\hline 0 & 0.0000 & 0.0230 \\
\hline 5 & 0.0159 & 0.0244 \\
\hline 10 & 0.0377 & 0.0303 \\
\hline
\end{tabular}

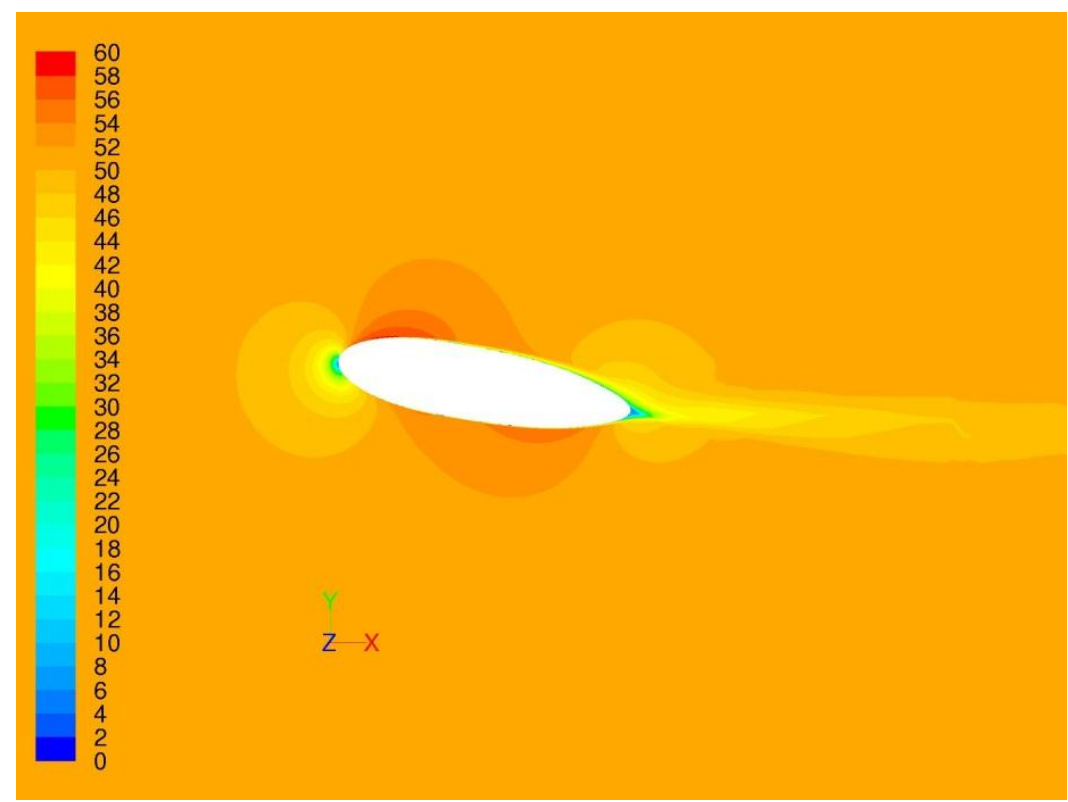

Figure 4-1: Velocity Magnitude (m/s) Contour Plot of 1:75 Scale YEZ-2A Airship Hull at $10^{\circ}$ AoA

\subsubsection{Axisymmetric Models}

Axisymmetric modeling provided a simplified approach to evaluate specific CFD particularities. The analysis was computationally efficient and offered an effective way to analyze mesh and model alterations within the CFD models. The approach described in Chapter 3 was utilized to construct several axisymmetric grids for sensitivity studies, designed to be geometrically similar to those used in the three dimensional study. Axisymmetric models were only evaluated for these studies due to their rotational axis which limits modeling to $0^{\circ}$ AoA only.

Three axisymmetric models with various levels of grid refinement were generated for the 1:75 scale hull and again for the full scale hull for use in a grid independence investigation. Grid 
independence utilized data from the convergence of second order steady state iterations of axisymmetric models. Resulting aerodynamic coefficients and their corresponding grid resolutions were subjected to the Grid Convergence Method [31] based on the Richardson extrapolation method. Specifically the variation of drag coefficient between three grid resolutions was tracked as a critical variable. A function of average cell area determined a representative cell size for each mesh. Essentially the representative size equaled the average cell size to the half power. This power was determined based on the axisymmetric nature of each grid. The grid refinement factor compared the resolution between two grids and equaled the representative size of the courser grid divided by the finer grid's value. Table 4-2 and Table 4-3 describe the three grids used in the small and full scale studies, respectively. Extrapolated coefficients and relative errors were then determined via iterative convergence of dependent functions of these variations. The study determined the necessary level of grid refinement in the model to produce an independent solution while minimizing computational expenses. The study was performed on both the scaled and full size axisymmetric hulls yielding an appropriate model for each case.

Table 4-2: 1:75 Scale Axisymmetric Grid Setup

\begin{tabular}{|r|l|r|c|r|c|}
\hline Mesh & Resolution & Cells & $\begin{array}{c}\text { Average } \\
\text { Cell Area }\end{array}$ & $\begin{array}{c}\text { Representative } \\
\text { Cell Size }\end{array}$ & $\begin{array}{c}C_{D} \text { (Tracked } \\
\text { Variable) }\end{array}$ \\
\hline 1 & fine & 18000 & 0.028445 & 0.17 & 0.02283 \\
\hline 2 & medium & 12000 & 0.042666 & 0.21 & 0.02283 \\
\hline 3 & course & 8000 & 0.063988 & 0.25 & 0.02286 \\
\hline
\end{tabular}

Table 4-3: Full Scale Axisymmetric Grid Setup

\begin{tabular}{|r|l|r|r|r|c|}
\hline Mesh & Resolution & \multicolumn{1}{c|}{ Cells } & $\begin{array}{c}\text { Average } \\
\text { Cell Area }\end{array}$ & $\begin{array}{c}\text { Representative } \\
\text { Cell Size }\end{array}$ & $\begin{array}{c}C_{D} \text { (Tracked } \\
\text { Variable) }\end{array}$ \\
\hline 1 & fine & 197400 & 14.59 & 3.82 & 0.01451 \\
\hline 2 & medium & 141000 & 20.43 & 4.52 & 0.01438 \\
\hline 3 & course & 98700 & 29.18 & 5.4 & 0.01430 \\
\hline
\end{tabular}

Results of the grid convergence study performed on small scale and full scale axisymmetric models are summarized in Table 4-4 and Table 4-5, respectively. Appendix C also contains $y+$, shear stress, and pressure plots along the axial position of the hull for both models and each grid. The study determined the level of discretization error present in the CFD solution. 
Estimates were produced after extrapolating values from the medium and fine grids, and again using the course and medium grids; results are labeled in the following tables as 2-1 and 3-2, respectively. Error is dependent in part on grid quality, specifically how flow features are resolved by the grid. The fine-grid convergence index (GCI) is also reported in the tables. It measures the percentage computed values differ from the asymptotic numerical values. The GCI represents an error band indicating how much the solution would change with further grid refinement; a small value indicates the computation is within the asymptotic range. This study only estimates discretization error and cannot account for modeling errors [32].

Table 4-4: 1:75 Scale Grid Convergence Results with Error Estimates

\begin{tabular}{|l|r|r|}
\hline \multicolumn{1}{|c|}{ Meshes: } & \multicolumn{1}{c|}{$\mathbf{2 - 1}$} & \multicolumn{1}{c|}{$\mathbf{3 - 2}$} \\
\hline Grid Refinement Factor $\left(\mathrm{h}_{\text {course }} / \mathrm{h}_{\text {fine }}\right)$ & 1.22 & 1.22 \\
\hline Extrapolated $\mathrm{C}_{\mathrm{D}}$ & 0.02283 & 0.02283 \\
\hline Approximate Relative Error & $0.018 \%$ & $0.092 \%$ \\
\hline Extrapolated Relative Error & $0.004 \%$ & $0.022 \%$ \\
\hline Fine-grid Convergence Index & $0.005 \%$ & $0.027 \%$ \\
\hline
\end{tabular}

Table 4-5: Full Scale Grid Convergence Results with Error Estimates

\begin{tabular}{|l|r|r|}
\hline \multicolumn{1}{|c|}{ Meshes: } & \multicolumn{1}{c|}{$\mathbf{2 - 1}$} & \multicolumn{1}{c|}{$\mathbf{3 - 2}$} \\
\hline Grid Refinement Factor $\left(\mathrm{h}_{\text {course }} / \mathrm{h}_{\text {fine }}\right)$ & 1.18 & 1.2 \\
\hline Extrapolated $C_{D}$ & 0.01463 & 0.01444 \\
\hline Approximate Relative Error & $0.896 \%$ & $0.522 \%$ \\
\hline Extrapolated Relative Error & $0.871 \%$ & $0.469 \%$ \\
\hline Fine-grid Convergence Index, GCl & $1.098 \%$ & $0.589 \%$ \\
\hline
\end{tabular}

The small scale study yielded an extrapolated relative error of $0.004 \%$ and a GCI of $0.0051 \%$ for the fine and medium grids. These values are low enough that their error bands will exceed the accuracy of the computed data, meaning the extrapolated drag coefficient of 0.02283 may differ by a negligible amount from the asymptotic value. The values corresponding to the medium and course grids also indicate an insignificant range for the error band. Comparing the two GCIs and apparent order can confirm that the computed values are within the asymptotic range of convergence, shown in Equation 4-1 (where $r$ is the grid refinement factor and $\mathrm{p}$ the apparent order). 


$$
\frac{G C I_{32}}{r^{p} G C I_{21}} \approx 1
$$

Evaluating this equation yields a value of 1.000, confirming the values are within the asymptotic range. These numbers indicate the medium mesh can adequately resolve flow conditions to yield coefficients with a low level of discretization error, making it suitable for successive analysis. One may also infer to a lesser degree that the uncertainty of spatial discretization for the geometrically similar 3D scaled models is reduced as well.

The full scale study produced error bands of +/- 0.00016 and +/- 0.00008 for the extrapolated drag coefficients of 0.01463 and 0.01444 , respectively. However, resulting GCI values do not satisfy Equation 4-1 and cannot verify that the computed values lie within the asymptotic range of convergence. This may be due to the use of a low grid refinement factor which Celik, et al. [31] suggest a value greater than 1.3 based solely on experience. They also note that a tracked variable difference very close to zero between grids, as is the case here, can adversely affect the procedure. This small difference may be an indication of oscillatory convergence or in some instances that the exact solution has been achieved. Despite the validation shortcomings, the study did yield extrapolated coefficients with relative errors below $0.1 \%$, suitable enough for performing grid selection. These results suggested that the medium mesh could yield data with an acceptable level of discretization error while minimizing computational expenses, making it suitable for ensuing analysis of both scale sizes.

\subsubsection{Transient Investigation}

After achieving grid independence a temporal sensitivity study was performed on selected axisymmetric models. This study performed a transient analysis of both medium meshed models over three different time steps. First an appropriate time step was determined for small and full scale axisymmetric grids. Its value was determined by examining wall-adjacent cells for the highest local flow velocity and the corresponding longitudinal cell length where it occurs. Dividing that cell dimension by the local velocity produced a time step that would limit fluid particles to travel less than one cell per time step.

Iterations were then performed under first order analysis until exhibiting convergence

under steady state conditions. The small scale model was initialized with 1000 first order steady state iterations while full scale model initialization required 3000 first order steady state iterations with solution controls relaxed by $20 \%$. These relaxation numbers are under-relaxation 
factors for each equation solved within the pressure-based solver. At each iteration these factors control the update of computed variables. Their default values are optimal for the largest number of possible cases. But for some nonlinear problems like turbulent flows, these factors must be initially relaxed to avoid divergent or unsteady behavior [29].

A second order implicit transient analysis was then performed on each model, with their respective time steps, until convergence was achieved. To evaluate the solution's sensitivity to the time step, further transient analysis was performed on initialized models, this time using time steps corresponding to $1 / 10$ and 10 times the original values. The results of the six transient simulations are shown in Table 4-6 and indicate that the solutions are independent of the time step. Plots available in Appendix $\mathrm{C}$ show the drag coefficient history for each time step versus flow time. Furthermore, there exists a negligible difference between steady state and transient results, thus indicating the steady flow solver is adequate for such analysis, again conserving computational expenses. These axisymmetric studies were however limited to $0^{\circ} \mathrm{AoA}$ cases and could not examine higher angles where a stronger need for unsteady simulation may exist.

Table 4-6: Results of Transient Sensitivity Study Performed on Axisymmetric Medium Mesh Grids

\begin{tabular}{|r|r|r|r|r|r|r|}
\hline & \multicolumn{3}{|c|}{ Model Scale } & \multicolumn{3}{c|}{ Full Scale } \\
\hline$\Delta \mathrm{t}(\mathrm{s})$ & 0.00004 & 0.0004 & 0.004 & 0.00135 & 0.0135 & 0.135 \\
\hline Flow Time $(\mathrm{s})$ & 0.2 & 0.48 & 0.4 & 10.7 & 27 & 27 \\
\hline Final $C_{\mathrm{D}}$ & 0.022834 & 0.022834 & 0.022834 & 0.014208 & 0.014305 & 0.014305 \\
\hline Time Averaged C & 0.022887 & 0.022856 & 0.022861 & 0.013384 & 0.013935 & 0.013941 \\
\hline
\end{tabular}

\subsubsection{D Full Scale Models}

The three dimensional modeling procedures, discussed in Chapter 3, were then applied to generate a full scale hull model for three different angles of attack $\left(0^{\circ}, 5^{\circ}\right.$ and $\left.10^{\circ}\right)$. A $15.43 \mathrm{~m} / \mathrm{s}$ flight speed and a corresponding Reynolds number of $1.36 \times 10^{8}$, based on airship length, constituted the flight conditions selected for the full scale setup. Grid geometry was matched to the medium mesh of the axisymmetric study and modeling setup mirrored the 1:75 scale three dimensional models. Analysis was initialized with 1000 first order, implicit, steady state iterations, before executing second order, implicit, steady state iterations until achieving convergence.

The resulting aerodynamic coefficients for each model are shown in Table 4-7. Additional data is displayed in Appendix B, including contour plots of velocity magnitude, 
pressure coefficient and shear stress for each AoA setup. The contour plots adequately represent expected flow physics, thereby suggesting the model and setup are appropriate selections for this application.

Table 4-7: 3D Full Scale Model Lift and Drag Coefficients

\begin{tabular}{|c|c|c|}
\hline $\begin{array}{c}\text { AoA } \\
\text { (degree) }\end{array}$ & $\mathrm{C}_{\mathrm{L}}$ & $\mathrm{C}_{\mathrm{D}}$ \\
\hline 0 & 0.0001 & 0.0144 \\
\hline 5 & 0.0121 & 0.0154 \\
\hline 10 & 0.0264 & 0.0191 \\
\hline
\end{tabular}

\subsection{Data Comparison}

\subsubsection{Modeled Flow Physics}

Scaled model testing performed by Gomes [4] was restricted by scaling factors and wind tunnel limitations eliminating any possibility of preserving Reynolds numbers between model scale $\left(6 \times 10^{6}\right)$ and full scale airships $\left(1.36 \times 10^{8}\right)$. To account for the discrepancy in Reynolds numbers surface roughening techniques were implemented as a way to simulate full scale flow conditions at a much lower Reynolds number.

In accordance with the experimental testing, the CFD models were analyzed using the wind tunnel Reynolds numbers. Scaled CFD model flow corresponded to that of the experimental setup, with regard to Reynolds numbers, and full sized model flow related to full scale flight conditions. While both scale models (CFD and experimental) were subjected to identical flow conditions, several discrepancies exist between the resulting flow physics. The conditions generated in the wind tunnel were dictated by altering the model's level of surface roughness. The level was determined when resulting aerodynamic coefficients matched available flight test data. In a similar fashion the scaled CFD hull was analyzed using various turbulent models and various turbulent intensity values. The selected setup was then determined by matching computed coefficients to experimental ones. Overall wind tunnel testing relied on surface roughening to trip the boundary layer and create the appropriate level of turbulence; the CFD approach relied on modeling parameters to control the fully turbulent flow around the smooth airship model [5]. 


\subsubsection{1:75 Scale}

The scale model lift and drag coefficients from the Gomes experiment, the previous WVU study, and this investigation are compared in Figure 4-2 and Figure 4-3, respectively. The coefficients from this study show good agreement with the wind tunnel data at the $0^{\circ}$ AoA but diverge with the increasing angle. The difference can be attributed to the hull-only setup of this investigation while Gomes incorporated fins and a gondola with his model. At the $0^{\circ}$ AoA, the fins have a minimal frontal profile adding little to the drag. As the angle is increased, however, a larger wake region develops and pressure drag increases, leading to a larger drag coefficient. The lift coefficient follows a similar trend, increasing due to fin interactions which alter the boundary layer and flow field in the airship's aft end. The interaction generates a larger area of suction pressure in the top aft portion and reduces the low pressure region in the bottom aft portion which generates lift. 


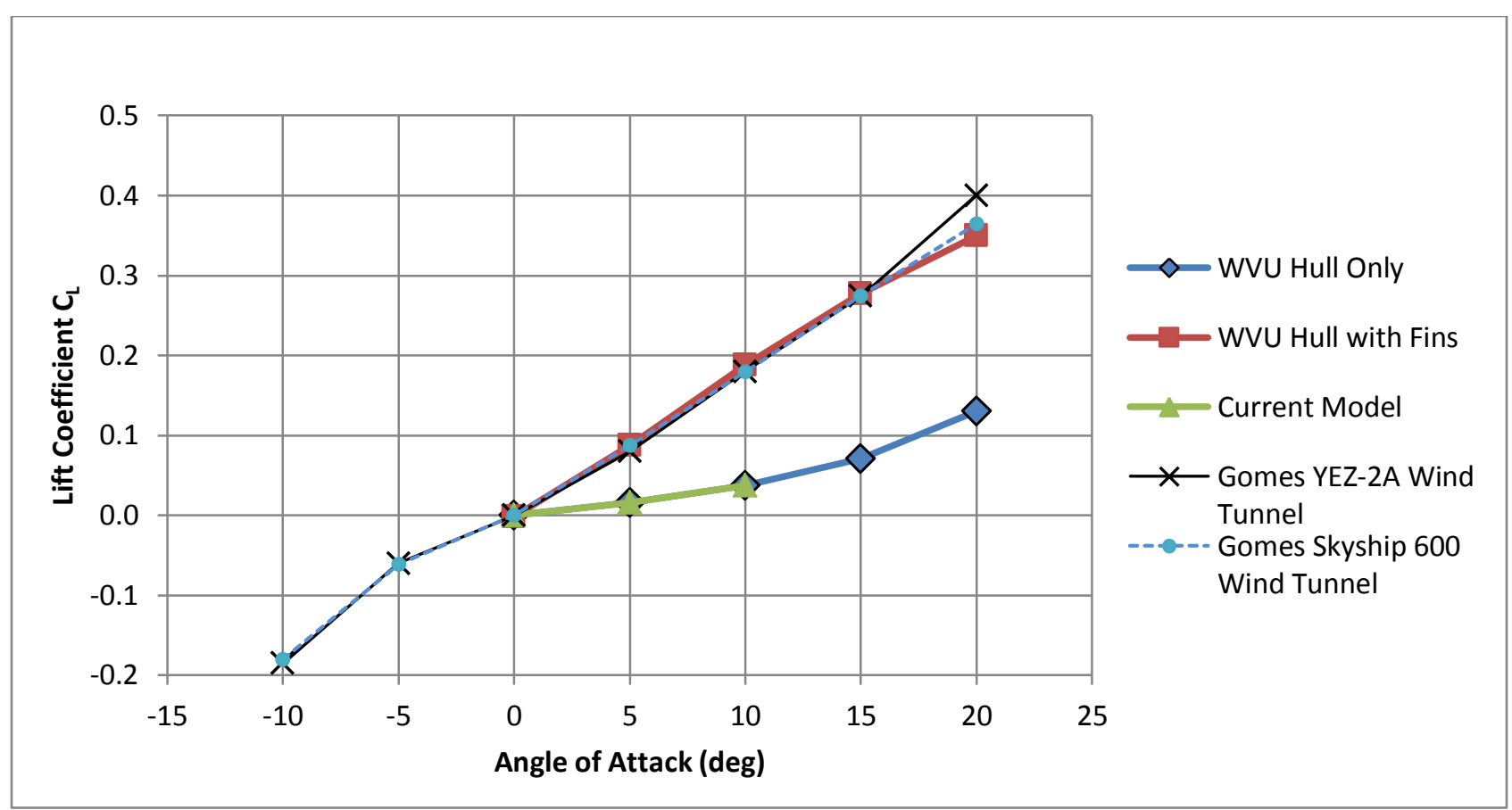

Figure 4-2: Lift Coefficient Comparison between 1:75 Scaled CFD and Wind Tunnel Models of the YEZ-2A and Skyship 600 Airship

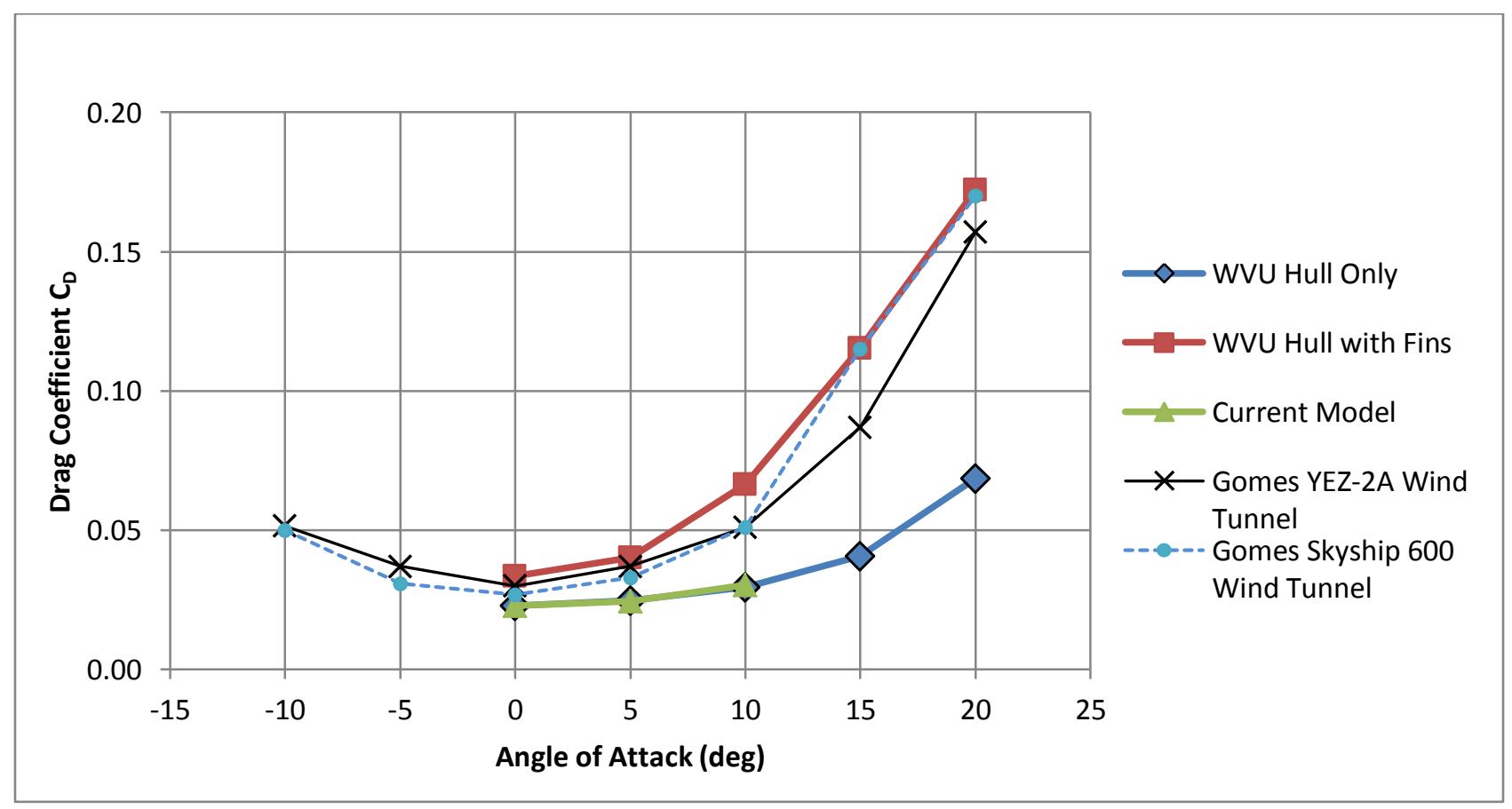

Figure 4-3: Drag Coefficient Comparison between 1:75 Scaled CFD and Wind Tunnel Models of the YEZ-2A and Skyship 600 Airship

The previous WVU study considered two model geometries, a hull with fins model which showed good agreement to the Gomes data and a bare hull model. The results of the current 
study show good agreement to the hull only data. As expected both coefficients increase with larger angles of attack at a rate much lower than the hull with fins model. The data indicate just how critical fin interactions can be to overall aerodynamics of the airship and their increasing contributions relative to increasing angle of attack.

\subsubsection{Full Scale}

Full scale lift and drag coefficients for three angles of attack $\left(0^{\circ}, 5^{\circ}\right.$ and $\left.10^{\circ}\right)$ are shown in Figure 4-4 and Figure 4-5, respectively, as compared to the 1:75 scale CFD models. Results follow the same trends exhibited by the scaled models, increasing with angle of attack. However, coefficients are lower than scale model values. As expected lift coefficients show zero lift with a zero degree AoA for all models. Increasing the AoA provides an increase relative to lift coefficients of both sized models. Full sized lift coefficients increase at a lower rate and the data diverges between models with increasing angles. Drag coefficients follow the same trends between models but full scale values are lower with around a 45\% consistent difference.

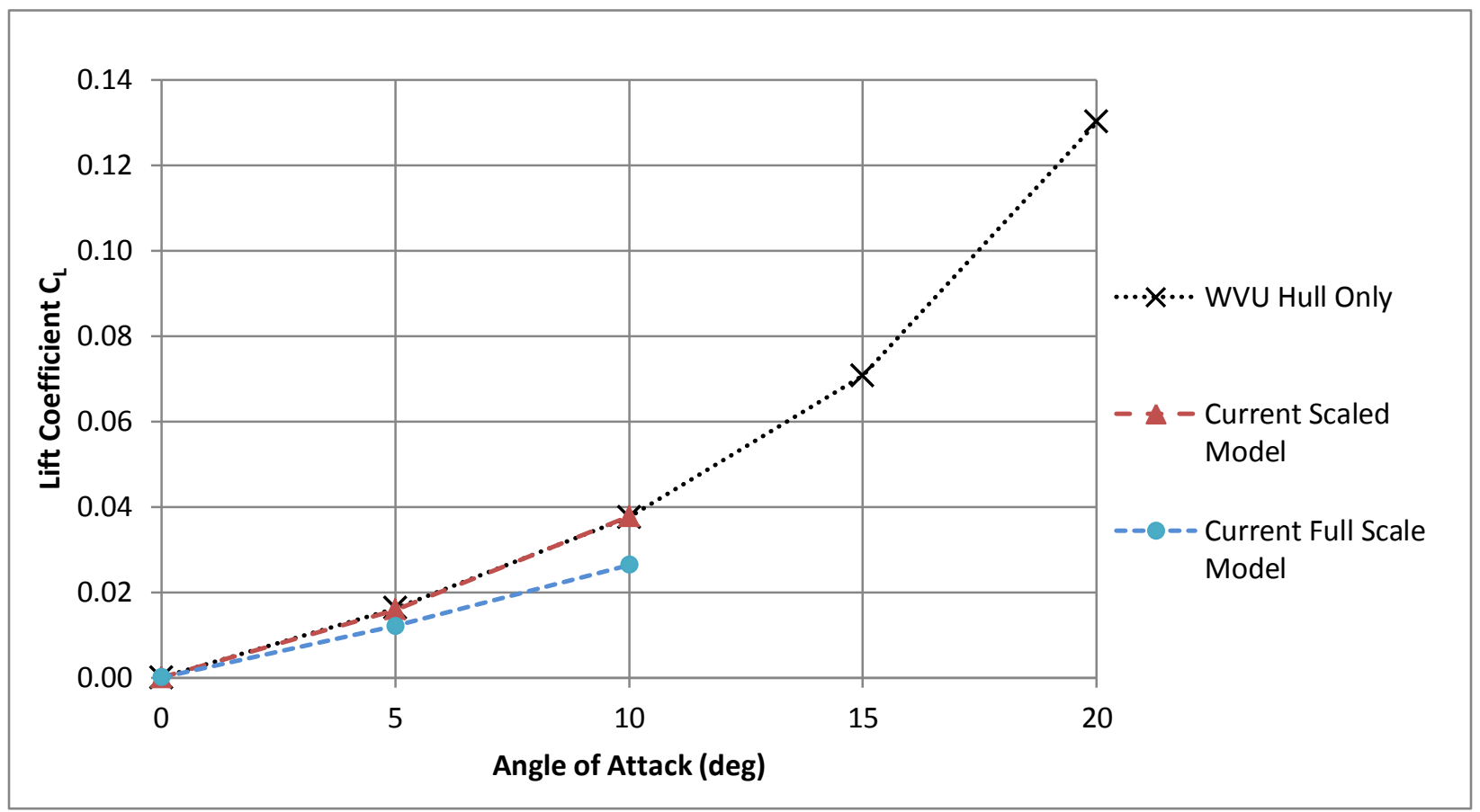

Figure 4-4: Lift Coefficient Comparison of 1:75 and Full Scale YEZ-2A CFD Models 


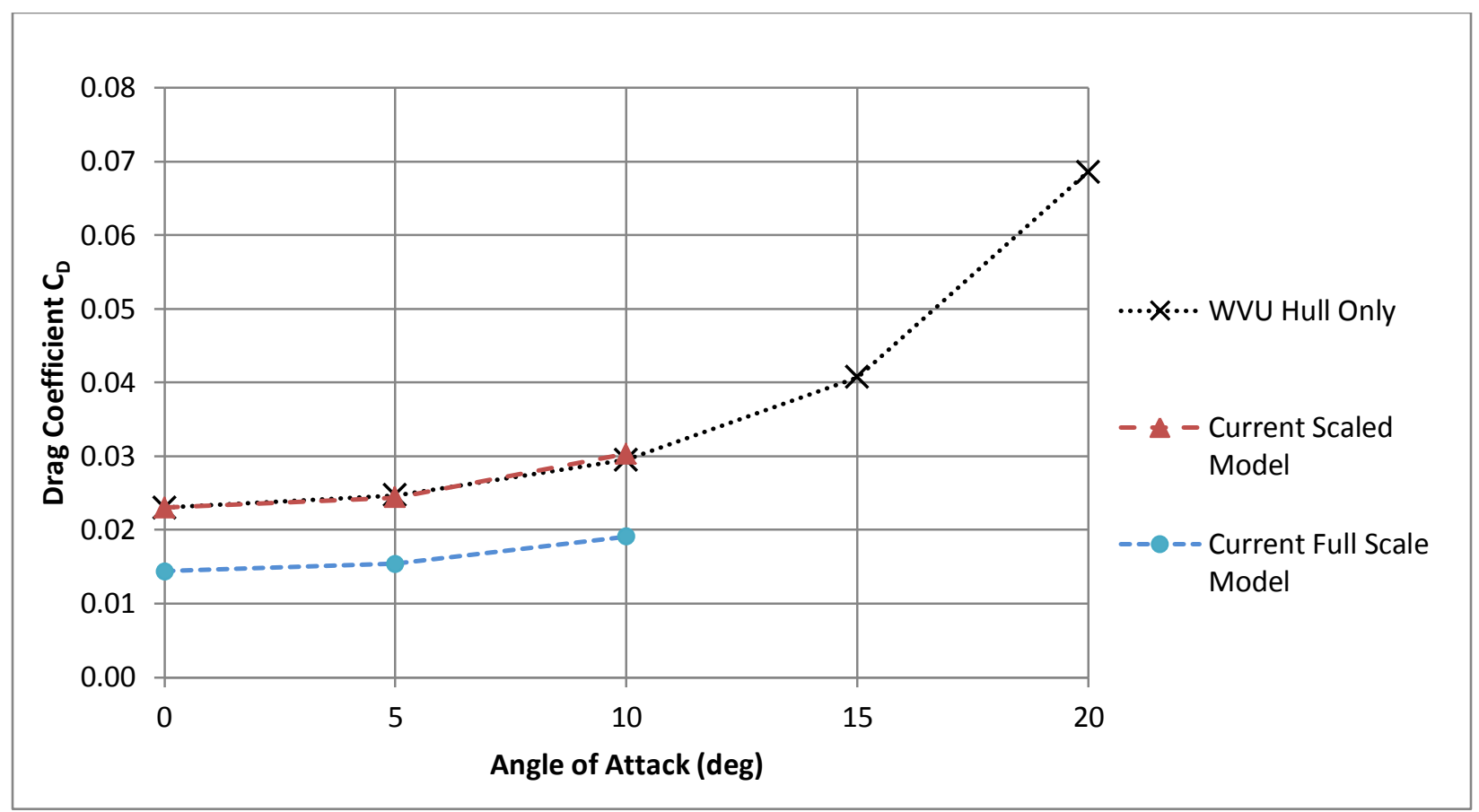

Figure 4-5: Drag Coefficient Comparison of 1:75 and Full Scale YEZ-2A CFD Models

\subsection{Full Scale Implications}

An ultimate goal of any scaled testing is to provide insight into full scale conditions, but the validity of what that modeling process may infer must first be scrutinized. Wind tunnel testing was conducted for aerodynamic considerations, mainly to predict the response of the YEZ-2A airship in actual flight conditions. Several challenges were encountered during wind tunnel testing which inhibited Reynolds number preservation, thereby causing uncertainty in any full scale predictions.

Current CFD investigations included full scale analysis to provide comparisons and understanding for the scaled modeling by exploring what factors are influential to the results and why. Full scale coefficients generated from the CFD analysis were lower than model scale values, differing by around 45\%. Examining additional test results and statistical functions can aid in confirming whether such a difference is appropriate or not.

The increased Reynolds number flow generated by full scale conditions produced lower aerodynamic coefficients as discussed by Hoerner [3]. For a three dimensional streamlined body like that of an airship hull, boundary layer transition is a function of shape, Reynolds number and free stream turbulence, among other factors. This relationship is shown in Figure 4-6, which 
compares experimental and semi-empirical drag coefficients versus Reynolds number for bodies with different fineness ratios (1/d) [3]. The experimental data is locally plotted below $10^{7}$ in a scattered fashion indicating an unpredictable regime centered above $10^{6}$. This region is comprised of data relating to the laminar to turbulent transition phase of the streamlined bodies. Within this transition phase there exists a critical Reynolds number where the drag coefficient experiences a significant drop usually reaching its minimum.

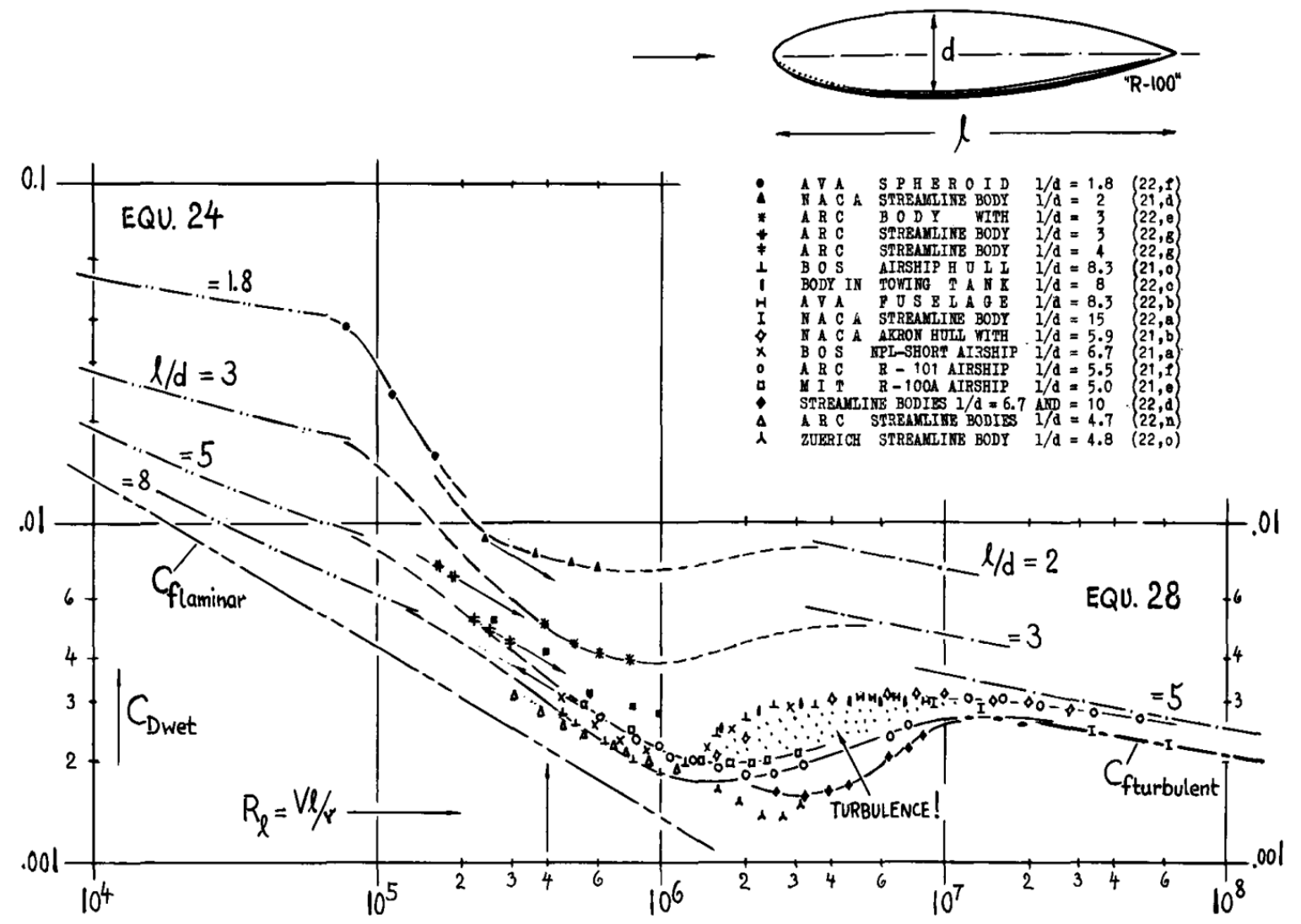

Figure 4-6: Drag Coefficients Based on Wetted Area versus Reynolds Number for Several Airships with Various Fineness Ratios (1/d) [3]

Experimental confirmation is difficult in the transitional region especially for three dimensional objects. Transition along a body of revolution occurs in an unpredictable manor. Along the transition interval, drag coefficients of two bodies with the same thickness ratio at the same Reynolds number may differ. This may be a result of interference effects from mounting in the wind tunnel (supporting a 3D body is practically impossible without disturbing the flow pattern). Also a three dimensional body will generate smaller pressure minima. The boundary layer transition is therefore not fixed and becomes too complex for any practical correction 
factors. Beyond the transition range drag coefficients will increase slightly before declining further. At this point the coefficients align in a pattern that descends with greater Reynolds numbers. Drag values are still dependent on shape (1/d) but the slope of descent is essentially equal for rotationally symmetric bodies. This linear relationship results from the fully turbulent boundary layer; as it now controls total drag in proportion to turbulent skin-friction drag. These circumstances present a less complicated phase in Reynolds number flow still too complex for theoretical analysis but suitable for approximation with statistical functions [3].

Challenges accompanying transition can be avoided by considering a higher Reynolds number or by forcing boundary layer transition. Introducing turbulence by surface roughening or other means will force an early transition point, relocating the drag data to form the fullyturbulent linear relationship magnitudes lower than normal. This is often practiced in wind tunnel testing when modeling is physically restricted to the transitional region. Full scale values are then extrapolated with formulas. The sloped lines around $10^{7}$ in Figure 4-6 represent the predictions of such a formula for several thickness ratios. The plotted equation is based on experimental evidence but appears valid up to the highest Reynolds numbers likely encountered (around $10^{10}$ ). The semi-empirical function predicts drag on the basis of thickness ratio and turbulent skin friction drag coefficient, shown in Equation 4-2 [3].

$$
\begin{gathered}
C_{D A}=C_{f}\left(1+1.5\left(\frac{d}{l}\right)^{3 / 2}+7\left(\frac{d}{l}\right)^{3}\right) \\
C_{f}=\frac{K}{R e_{l}{ }^{1 / m}}
\end{gathered}
$$

Manipulating data to non-dimensional values is a fundamental technique for most scaled modeling. For airship design the process is primarily focused on predicting aerodynamic forces as they vary with flow conditions. The use of non-dimensional Reynolds number, drag and lift coefficients permits comparisons between two similar bodies (identical in shape, but different in size). For this research the models maintained an identical thickness ratio but differed in scale. The size difference did not directly alter any force coefficients except through the Reynolds number, since it is based on a model dimension.

Since the drag coefficient, which varies with Reynolds number, is a non-dimensional relationship, the statistical function is based only on the thickness ratio. Hence, drag values can be compared for different scales. Equation 4-2 computes drag coefficients using the thickness ratio with additional constants to show good agreement with experiments. The function is 
plotted in Figure 4-7 for $d / l=0.247$ along with the 1:75 and full scale CFD drag coefficients at $0^{\circ}$ AoA. The function uses Equation 4-3 with constants $K=0.030$ and $m=7$, values recommended by Hoerner [3] for this range of Reynolds numbers. These data match the slope of the statistical function but differ by around $13 \%$. The constants $K=0.032$ with $m=6.5$ or the ratio $d / l=0.165$ would better represent a line through the CFD data points. The discrepancy may be due to the turbulence generated through surface roughness in the wind tunnel, or likewise through the turbulent intensity factor in the CFD modeling. Additionally, this function (as well as the data plotted in Figure 4-6) was derived using early wind tunnels that were not able to adequately control free stream turbulence.

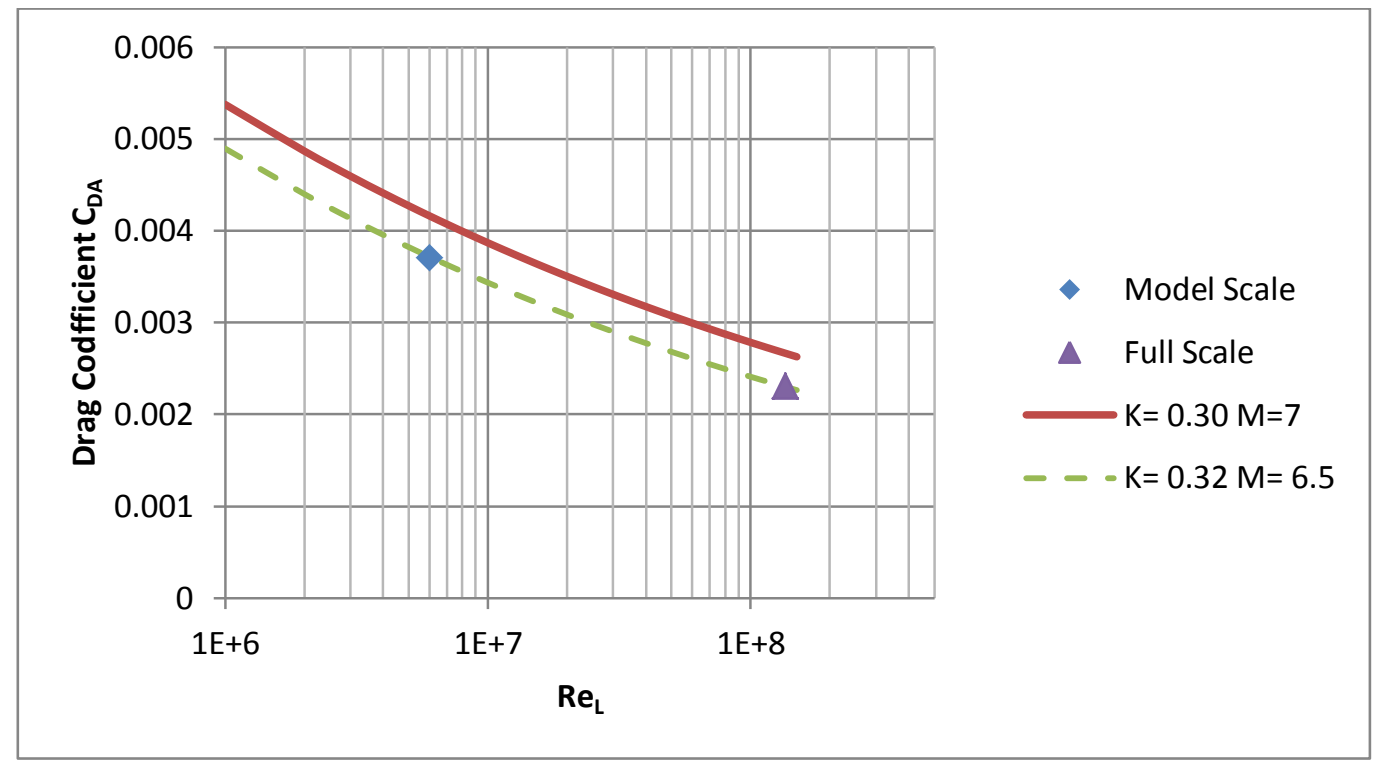

Figure 4-7: CFD Data with Equation 4-2 plotted for $d / l=0.247$

Overall, the CFD data matched the semi-empirical relationship extremely well in trend with a slight offset in magnitude. This is further evidence that the hull-only $\mathrm{C}_{\mathrm{D}}$ values are likely to exhibit a $45 \%$ difference between model and full scale coefficients. Consequently, using the scale model coefficients for a full scale design would lead to an over estimation of aerodynamic forces and potentially over-designing the structure. 


\section{Chapter 5 Summary and Recommendations}

\subsection{CFD as an Airship Design Tool}

Numerical analysis has progressed over the recent decades fostered by the emerging field of CFD. The exponential growth of computing power has propelled analysis from simple two dimensional laminar flows to fully turbulent three dimensional flows with separation. Pairing current computing power with modern CFD software offers a powerful tool for comprehensive analysis. As modeling capabilities increase, so do the potential for errors. Successful simulations and accuracy of conclusions drawn are at the mercy of the CFD user. Errors are minimized when suitable conditions, appropriate models and meshes, algorithms, and other inputs are determined by the user. CFD is still maturing and a universal set of modeling procedures does not exist. There are different guides and procedures available depending on the level of accuracy required. However, detailed assessment should include comparisons to theory and experiments for verification and validation if possible.

Airship design involves aerodynamic, fabrication and structural considerations. CFD analysis has been applied to several of these aspects. An airship hull is the largest and most important component and receives the greatest attention. In general most studies examine aerodynamic force coefficients to evaluate potential designs. This research has briefly explored the potential for CFD as an airship design tool. Results showed that force coefficients were generated with an acceptable level of accuracy using numerical analysis. Unlike most wind tunnel tests, numerical modeling can provide specific details about the flow and in any location of the flow field. Under certain circumstances the computational models can be easily altered to evaluate different designs or flight conditions. Also, computers are not physically restricted by model dimensions, permitting analysis into full scale designs.

Numerical analysis is becoming a major tool to evaluate an aircraft design. Many unconventional prototypes are being considered for modern airships, such as dual hulls or hybrid lift. These designs have seen little attention in past wind tunnel tests; the benefits of modern CFD make it the ideal choice for such analysis. CFD modeling has been extremely successful, but still shows problems modeling complex flows and often requires an enormous amount of computation time. Generating an aerodynamic data base for a large number of flow conditions is 
not yet practical with CFD analysis. Despite this, CFD can provide a numerical platform to obtain relevant insight into the important factors of airship design.

\subsection{Implications of Full Scale and Model Scale Results}

The research performed by Gomes yielded aerodynamic coefficients of the YEZ-2A airship for several designs and flight scenarios. However, the current research has shown the application of these coefficients into full scale flight analysis should be done with care and mindful of several considerations. First the YEZ-2A was a prototype airship; therefore, no actual flight test data was available for validation. As a result testing techniques were validated using only a single drag coefficient from a Skyship-600 (featuring an alternative fin configuration) flight test and its corresponding model. Also testing was performed under a Reynolds number flow on the order of $10^{6}$, two orders of magnitude lower than full scale flight.

Research performed here furthered the aerodynamic investigation into the YEZ-2A airship. Tasks included examining meshing requirements and determining viscous model parameters to yield a high fidelity model while minimizing computational expenses. Data was then compared between CFD and wind tunnel investigations with great consideration given to the different means of turbulence generation. While the wind tunnel testing relied on surface roughening to induce turbulence along the model, CFD analysis considered a smooth model subjected to fully turbulent flow. One major objective involved an examination of a full scale model with CFD analysis. Results have shown that full scale turbulent flight coefficients of an airship hull will not equal those from a scaled model whose Reynolds number is not matched. Statistical functions and experimental data were examined to evaluate the difference. Specifically a semi-empirical equation was used to confirm the Reynolds number trend using drag coefficients. This discrepancy resulted from the lack of Reynolds numbers similarity among models. This research was also concerned with developing a suitable approach for turbulence modeling around an airship. The computational approach was verified by examining potential errors through sensitivity studies. Numerical data was validated using experimental results.

With the afore mentioned considerations in mind the 1:75 scale CFD results showed good matching to the Gomes wind tunnel data at $0^{\circ}$ AoA. However, full scale CFD drag coefficients were proportionally lower. This condition was supported by a semi-empirical relationship. 
Applying scaled force coefficients to full size structural requirements will then incur an over estimation of forces for such a design and conditions. Performing various sensitivity studies and comparing this research with previous work aided in reducing uncertainty in the results and provided verification to the modeling process. Overall, the method was capable of generating qualitative results with a suitable level of accuracy.

\subsection{Further Research}

This study examined the basic aerodynamic response for two sizes of models over a few turbulent flight conditions. Several follow-up studies and investigations could be performed to continue this work. If the YEZ-2A airship is to develop further, studies should examine a broader range of flight conditions. Analysis should be extended to additional airship features, i.e. fins, control car, engines etc. Analysis should also include non-linear dynamics like added mass to fully investigate stability.

Research could be continued to provide improvements to the modeling process of this study. One might examine variations in modeling parameters and design alterations. Future work should involve more complex turbulent models as increases in computing performance permit. Alternate models could also be included such as LES or hybrid turbulent models. It may be important that any improvements to, or agreement of, universal guidelines for verification and validation re-examine this method, especially if a higher degree of accuracy is desired in future work. Also, these results could be further verified as data from future flight tests become available. 


\section{References}

1. War Department. Airship Aerodynamics. Honolulu, Hawaii : University Press of the Pacific, 2003 (Reprint of 1941 edition). ISBN: 1-4102-0614-9.

2. Burgess, C. Airship Design. Honolulu, Hawaii : University Press of the Pacific, 2004. ISBN: 1-41021173-8.

3. Hoerner, S. F. Fluid Dynamic Drag. Bakersfield, CA : by the author, 1965. Library of Congress Catalog Number 64-19666.

4. Gomes, S. B. V. An Investigation of the Flight Dynamics of Airships with Application to the YEZ-2A. Cranfield, UK : Cranfield Insitute of Technology, 1990. Ph.D. Dissertation.

5. Menchini, C., Huebsch, W. and Thompson, G. Computational Modeling of a New Surveillance Airship Design. Morgantown : West Virginia University, 2009. Final Report.

6. Saari, P. Science and Invention - Who Invented the Hot Air Balloon? History Fact Finder. [Online] 2001. <http://www.enotes.com/history-fact-finder/science-invention/who-invented-hot-airballoon>.

7. U.S. Navy. Kite Ballons to Airships... the Navy's Lighter-than-Air Experience. Naval Historical Center homepage. [Online] August 31, 1999. <http://www.history.navy.mil/branches/lta-m.html>.

8. Browne, M. U.S. Turns to Giant Blimp for Defense of the Nation's Shores. The New York Times. [Online] January 10, 1989. <http://www.nytimes.com/1989/01/10/science/us-turns-to-giantblimp-for-defense-of-the-nation-s-shores.html>.

9. Khoury, G. A. and Gillett, J. D. [ed.]. Airship Technology. Cambridge : Cambridge University Press, 1999.

10. Lutz, Th., Funk, P., Jakobi, A. and Wagner, S. Aerodynamic Investigations on Inclined Airship Bodies. Institute for Aerodynamics and Gasdynamics. Stuttgart, Germany : University of Stuttgart, 1998.

11. White, F. Viscous Fluid Flow. New York : McGraw Hill, 2006.

12. Kim, S., Rhee, S. and Coklijat, D. Fluent Newsletter. The Prolate Sphereoid Separates Turbulence Models. [Online] Spring 2005.

<http://www.fluent.com/about/news/newsletters/05v14i1/index.htm>.

13. Elert, G. Flow Regimes. The Physics Hypertextbook. [Online] 2010. <http://physics.info/>.

14. Jakobi, A., Funk, P., Lutz, Th. and Wagner, S. Calculation of Aerodynamic Forces on Inclined Airship Bodies - Boundary-Layer Calculation Method. Institute for Aerodynamics and Gasdynamics. Stuttgart, Germany : University of Stuttgart, 2000.

15. Young, D., Munson, B. and Okiishi, T. A Brief Introduction to Fluid Mechanics. York, Pa : John Wiley \& Sons, Inc., 2004. ISBN 0-471-66077-9.

16. Omari, K., Schall, E., Koobus, B. and Dervieux, A. Turbulence Modeling Challenges in Airship CFD Studies. Prensas Universitarias de Aargoza : Actes des VIII Journees Zargoza-Pau, 2004. pp. 545-554.

17. Jamison, L., Sommer, G. and Porche III, I. High-Altitude Airships for the Future Force Army. Santa Monica, CA : Rand Corporation, 2005. Technical Report. 
18. Eaton, E. Airships and the Modern Military. Carlisle Barracks, Pennsylvania : U.S. Army War College, 1991. USAWC Military Studies Program Paper.

19. Polmar, N. The Naval Institute Guide to the Ships and Aircraft of the U.S. Fleet. : Naval Institute Press, 2001.

20. Lockheed Martin. Persistent Threat Detection System (PTDS). [Online] 2010. <http://www.lockheedmartin.com/products/PTDS/>.

21. Lockheed Martin. Lighter-than-Air Programs Lifting Off in Akron. [Online] 2010. <http://www.lockheedmartin.com/ms2/features/lighter-than-air.html>.

22. Brook, T. Demand for Spy Balloons in Afghan War Hits High. USA Today. [Online] September 28, 2010. <http://www.usatoday.com/news/world/afghanistan/2010-09-27-spyballoons_N.htm?csp=usat.me>.

23. Omari, K., Schall, E., Guer, Y. and Amara, M. Numerical Study of Turbulent Flow Around a Generic Airship at High Angle of Attack. Paris-Cachan, France : 4th International Conference on Computational Heat and Mass Transfer, 2005.

24. Lockheed Martin. High Altitude Airship (HAA). [Online] [Cited: October 20, 2010.] <http://www.lockheedmartin.com/products/HighAltitudeAirship/index.html>.

25. The Patuxent Partnership. About Us, Programs. [Online] 2010. <http://paxpartnership.org/>.

26. Kanikdale, T. Optimization of Airship Envelope Shape using Computational Fluid Dynamics. Bombay : Indian Institute of Technology, 2004. Masters Thesis.

27. Li, Y. and Nahon, M. Modeling and Simulation of Airship Dynamics. Montreal : Journal of Guidance, Control, and Dynamics, Vol. 30 No. 6, 2007. AIAA.

28. Wang, X. L., Ma, Y. and Shan, X. Modeling of Stratosphere Airship. Orlando, Fl : 48th AIAA Aerospace Sciences Meeting Including the New Horizons Forum and Aerospace Exposition, 2010. AIAA 2010-38.

29. Fluent. Theory Guide, User's Guide. Ansys Fluent. [Online] January 2009. <www.fluentusers.com>.

30. Lanfrit, M. Best Practic Guidlines for Handling Automotive External Aerodynamics with FLUENT. Darmstadt, Germany : Fluent, 2005. Version 1.2.

31. Celik, I., Ghia, U., Roache, P., Freitas, C., Coleman, H. and Raad, P. Procedure for Estimation and Reporting of Uncertainty Due to Discretization in CFD applications. : Journal of Fluids Engineering, Vol. 130, 2008. ASME 078001-3.

32. Slater, J. Resources for CFD Verification and Validation. NPARC Alliance CFD Verification and Validation Web Site. [Online] NAS A, July 8, 2008. <http://www.grc.nasa.gov/WWW/wind/valid/resources.html>.

33. Bairstow, L. Applied Aerodynamics. New York : Longmans, Green and Co., 1920. 


\section{Appendix A 1:75 Scale Contour Plots}
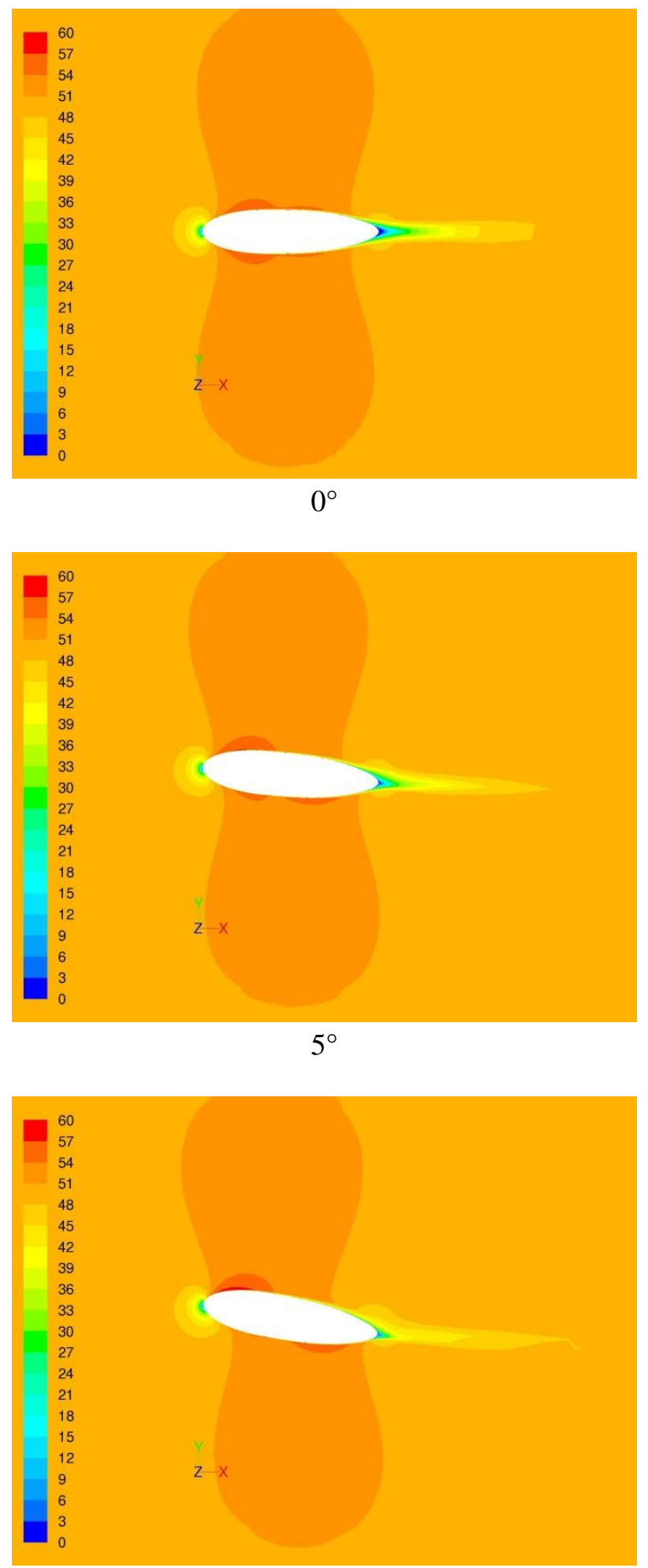

$10^{\circ}$

Figure A-1: Velocity Magnitude (m/s) Contour Plots of the YEZ-2A 1:75 Scale Hull-only CFD Model for Each Angle of Attack from the Longitudinal View 

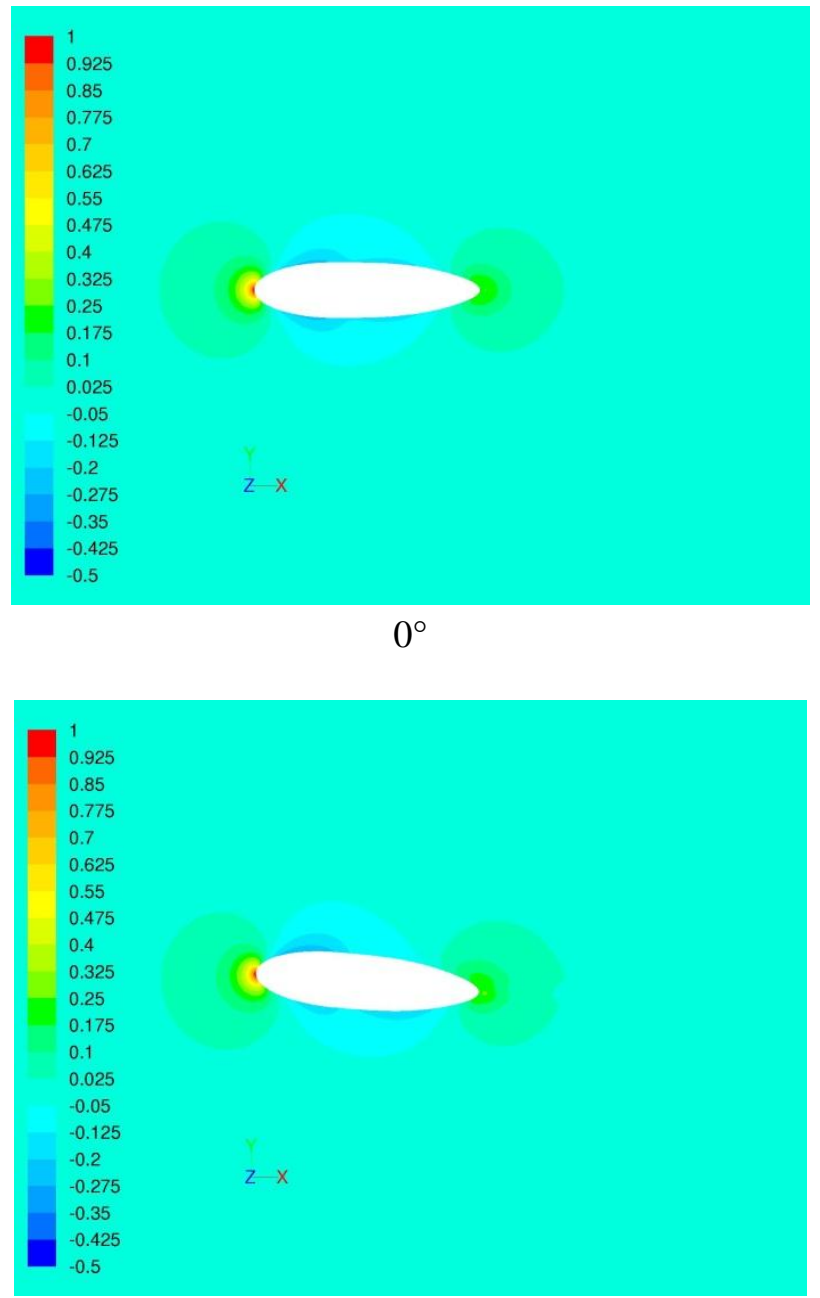

$5^{\circ}$

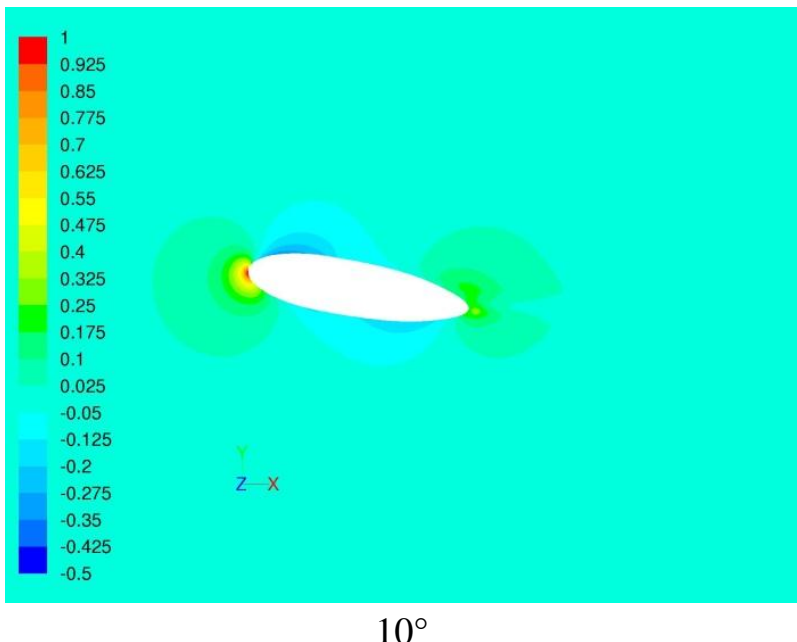

Figure A-2: Pressure Coefficient Contour Plots of the YEZ-2A 1:75 Scale Hull-only CFD Model for Each Angle of Attack from the Longitudinal View 

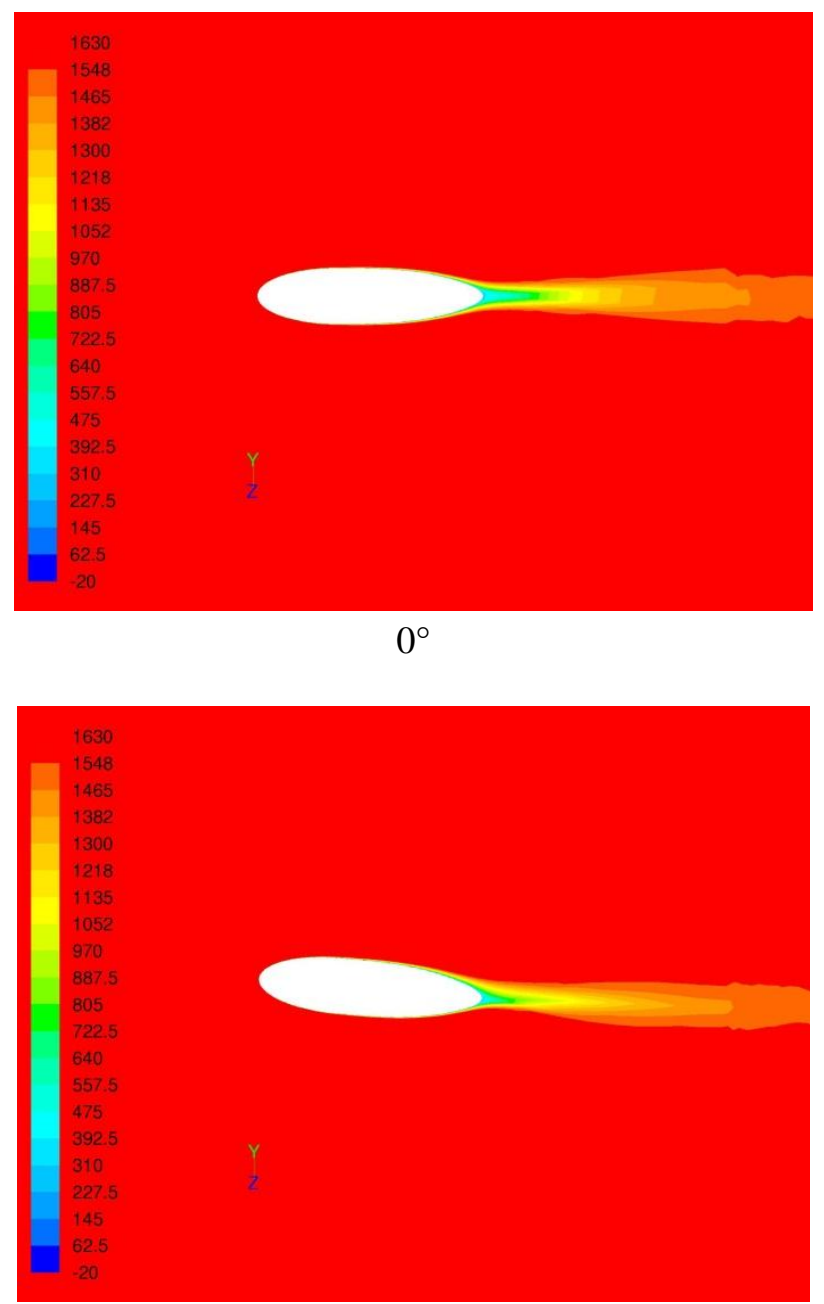

$5^{\circ}$

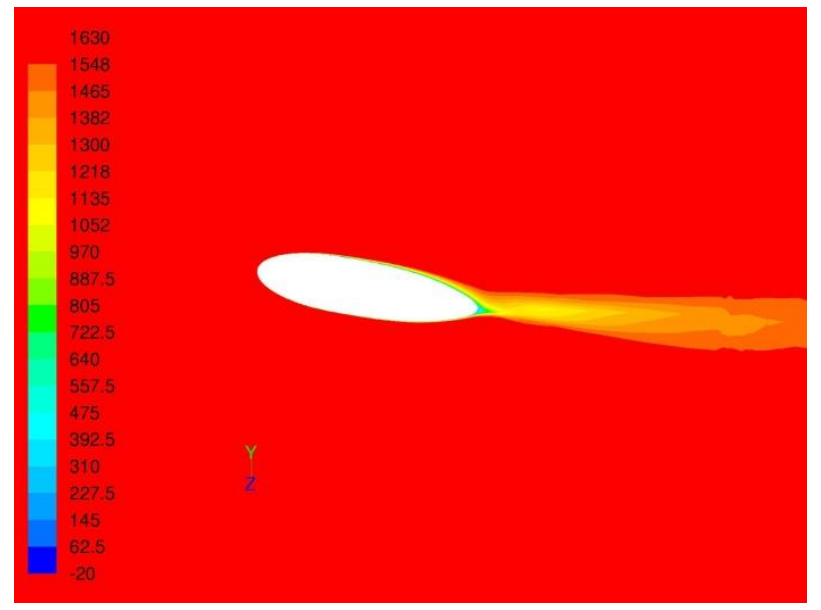

$10^{\circ}$

Figure A-3: Total Pressure (pascal) Contour Plots of the YEZ-2A 1:75 Scale Hull-only CFD Model for Each Angle of Attack from the Longitudinal View 


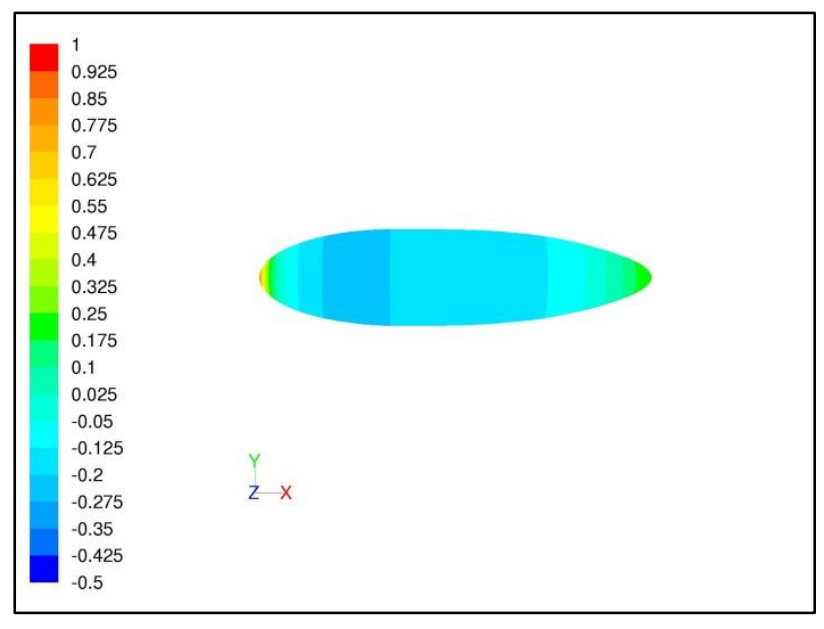

$0^{\circ}$

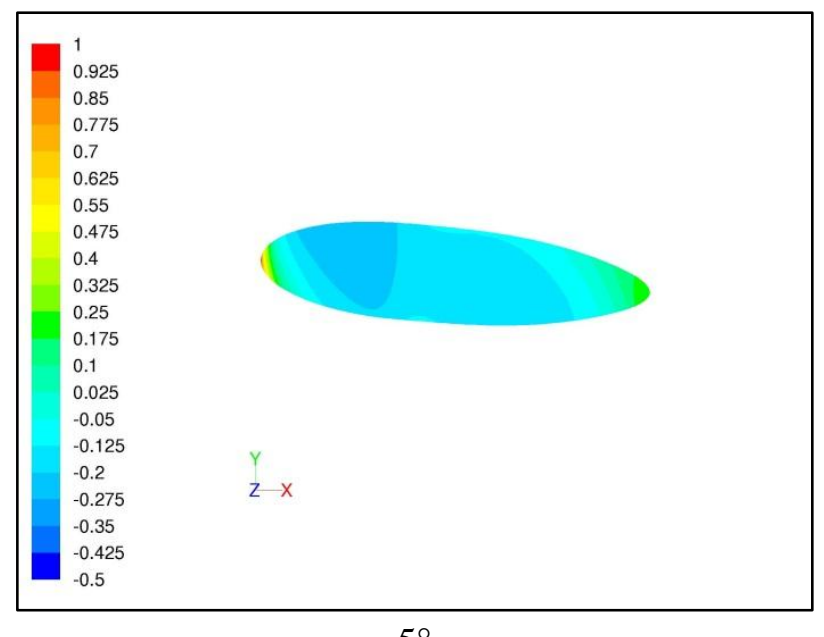

$5^{\circ}$

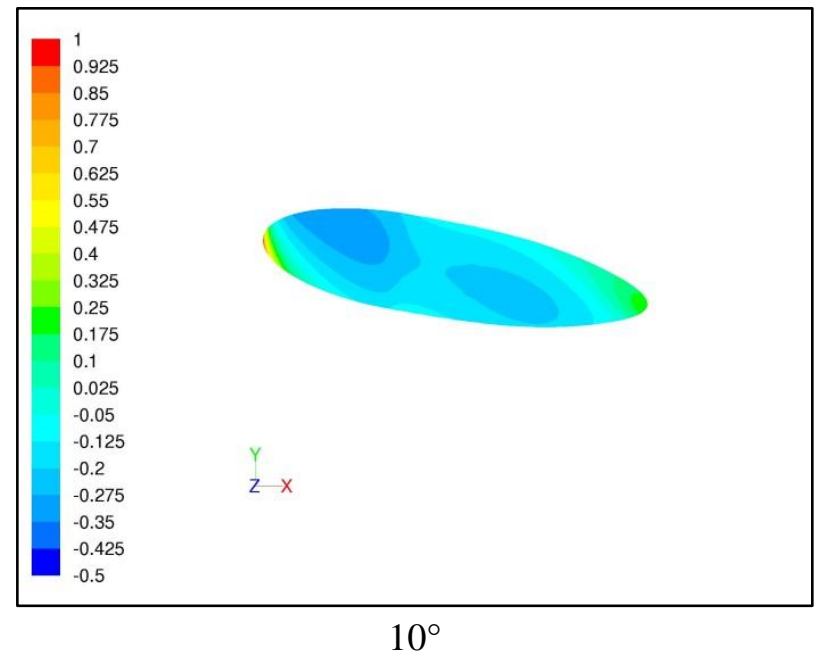

Figure A-4: Pressure Coefficient Contour Plots of the YEZ-2A 1:75 Scale Hull-only CFD Model for Each Angle of Attack from the Longitudinal View 

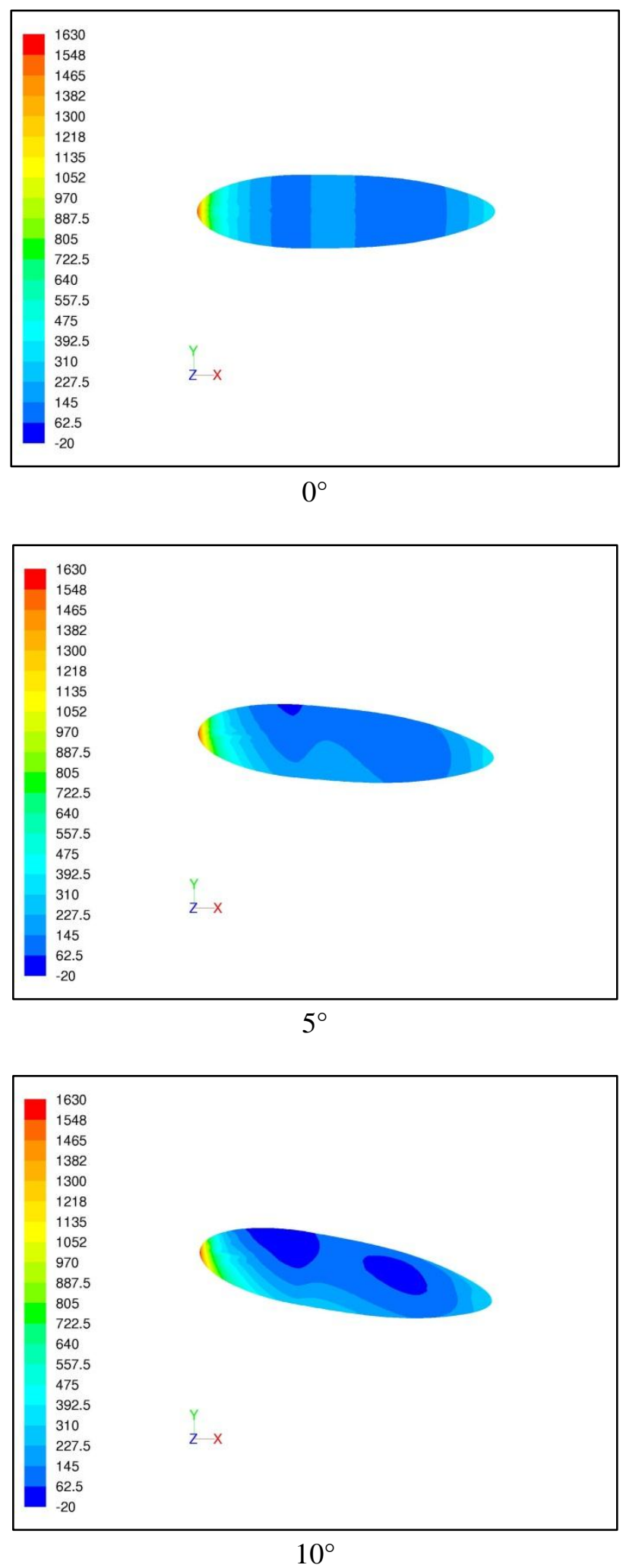

Figure A-5: Total Pressure (pascal) Contour Plots of the YEZ-2A 1:75 Scale Hull-only CFD Model for Each Angle of Attack from the Longitudinal View 

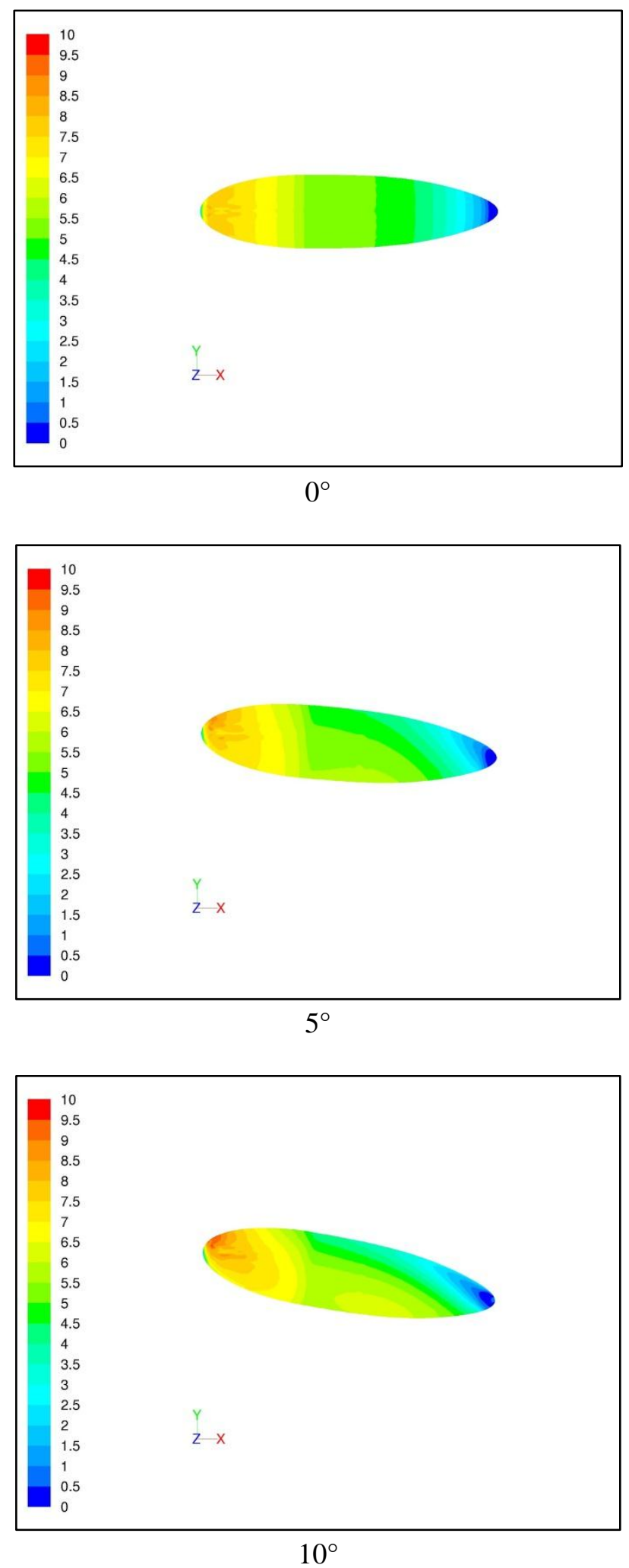

Figure A-6: Shear Stress (pascal) Contour Plots of the YEZ-2A 1:75 Scale Hull-only CFD Model for Each Angle of Attack from the Longitudinal View 

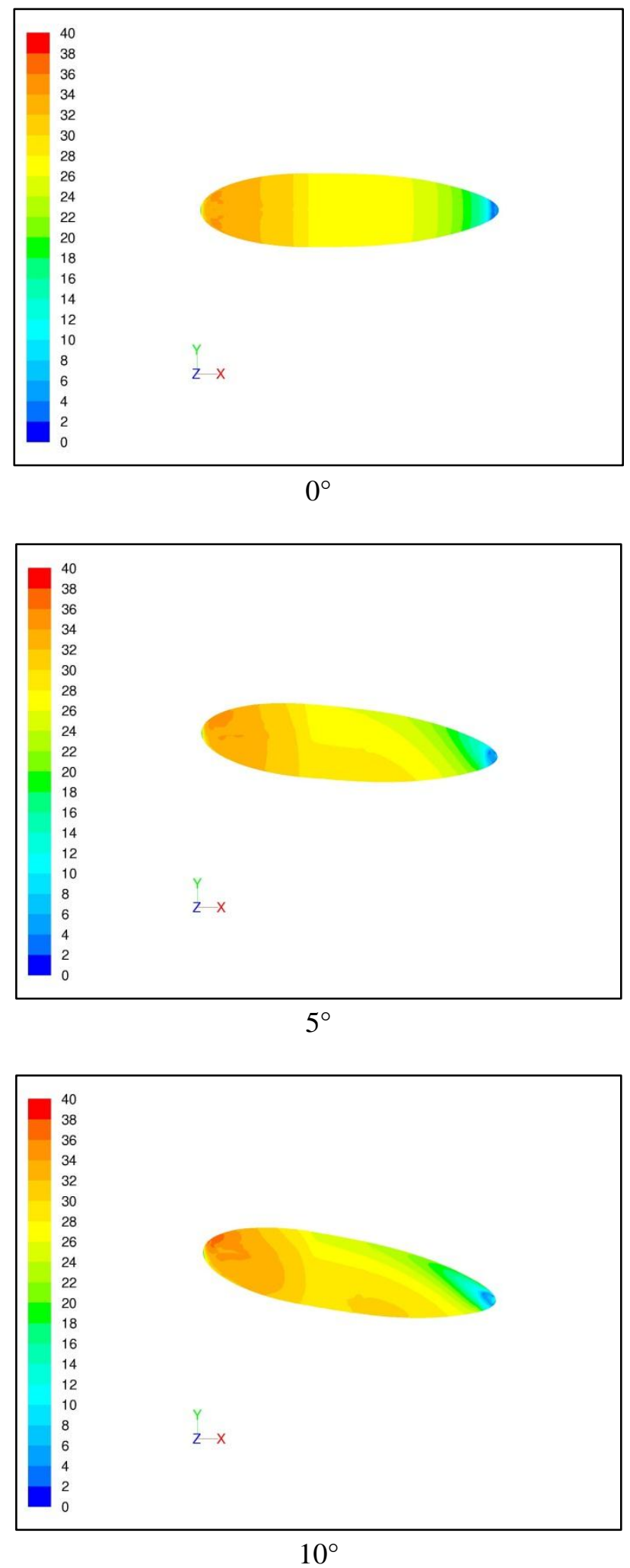

Figure A-7: y+ Contour Plots of the YEZ-2A 1:75 Scale Hull-only CFD Model for Each Angle of Attack from the Longitudinal View 


\section{Appendix B Full Scale Contour Plots}
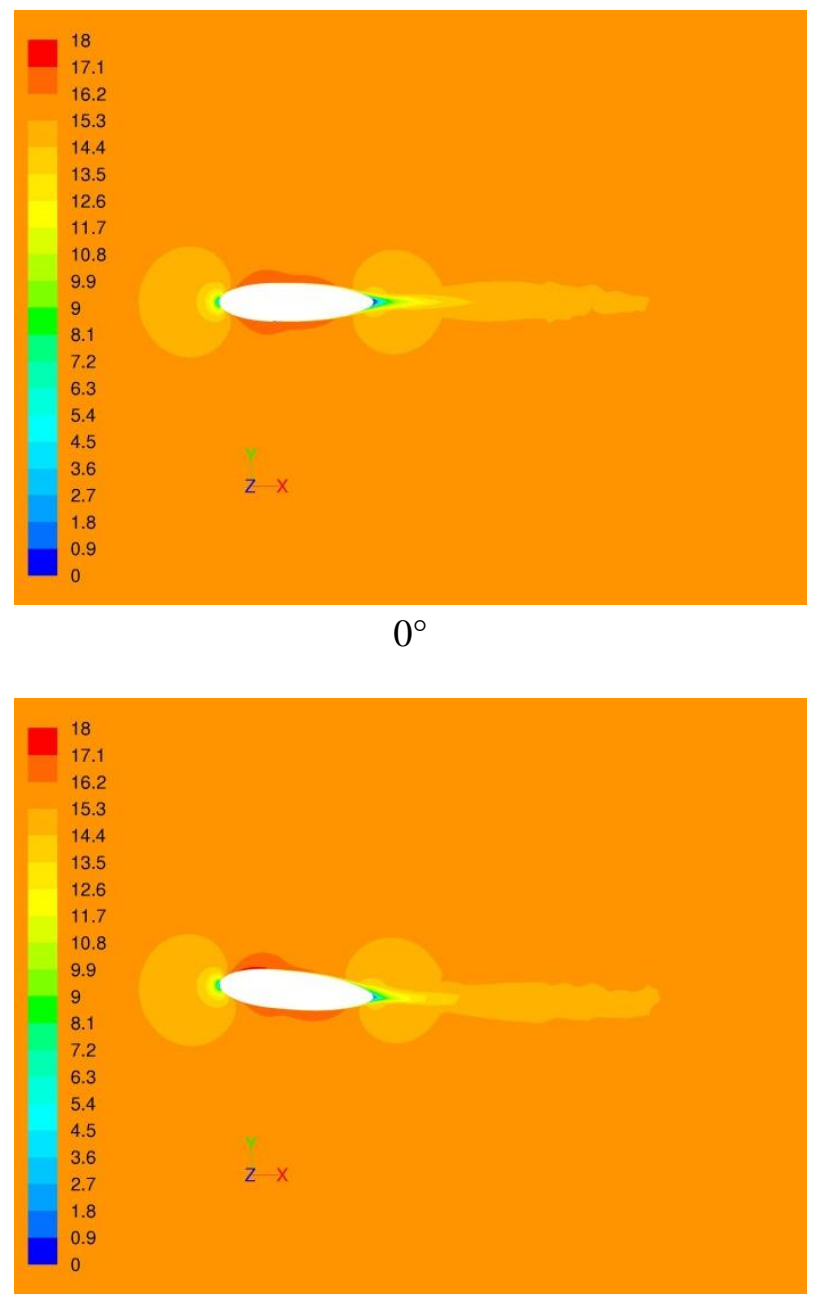

$5^{\circ}$

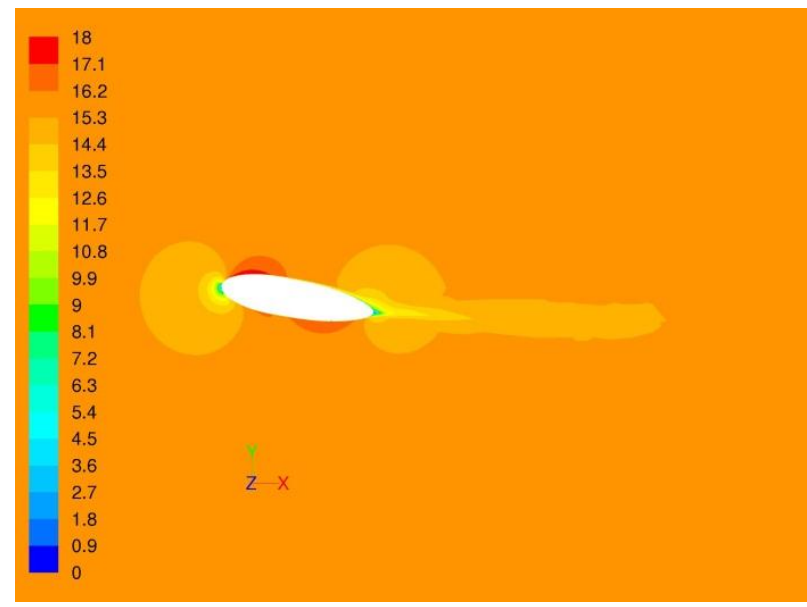

$10^{\circ}$

Figure B-1: Velocity Magnitude (m/s) Contour Plots of the YEZ-2A Full Scale Hull-only CFD Model for Each Angle of Attack from the Longitudinal View 

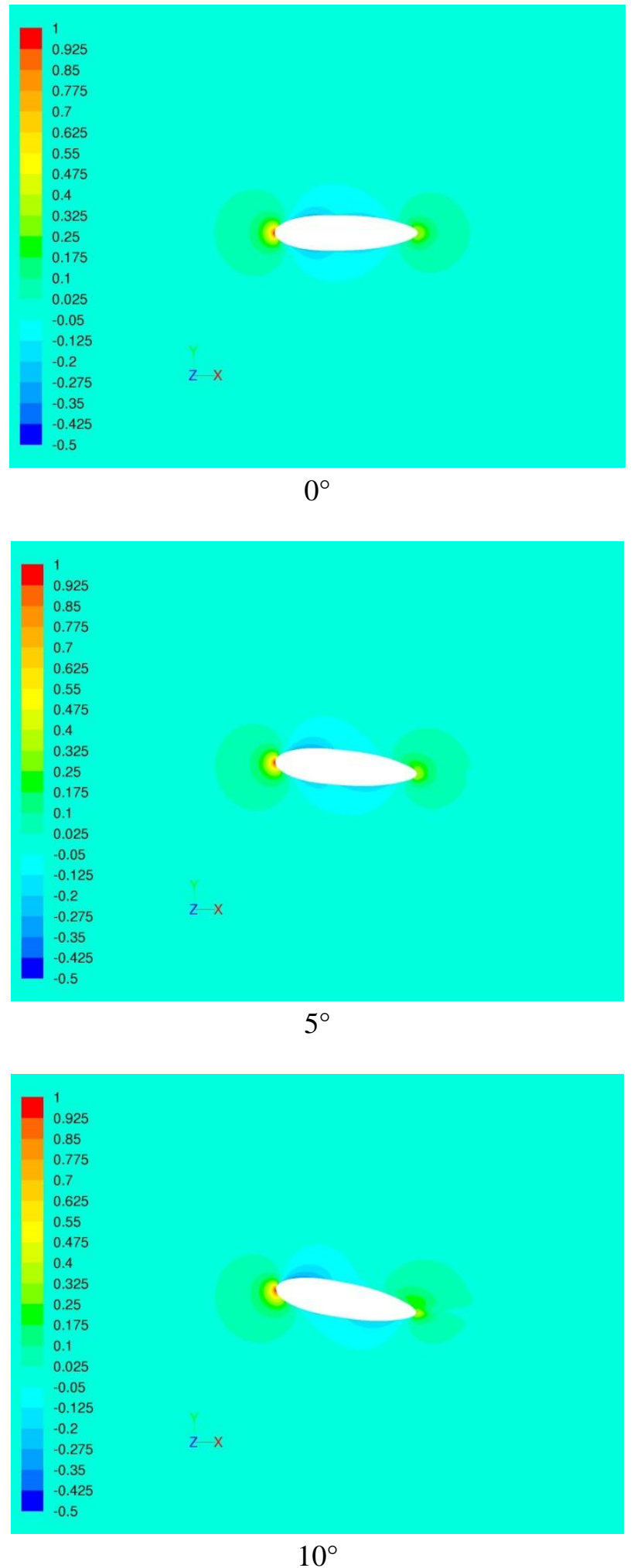

Figure B-2: Pressure Coefficient Contour Plots of the YEZ-2A Full Scale Hull-only CFD Model for Each Angle of Attack from the Longitudinal View 

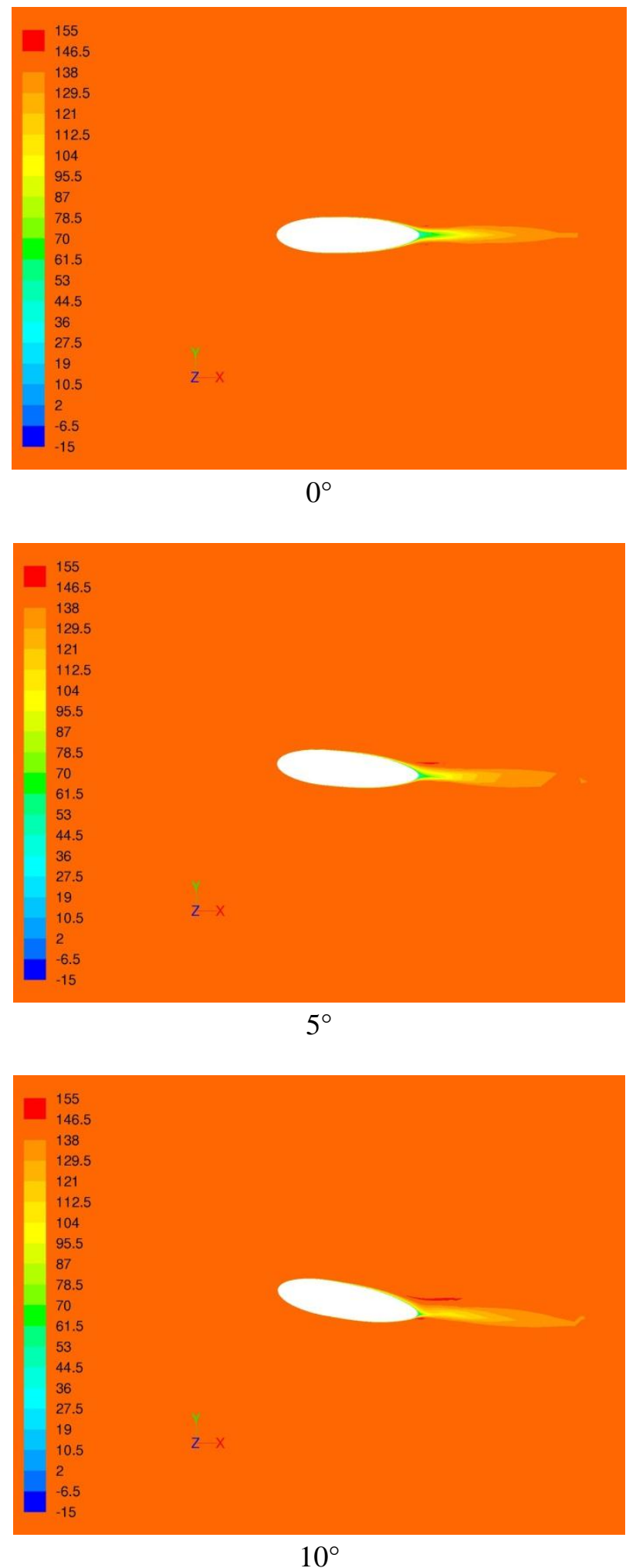

Figure B-3: Total Pressure (pascal) Contour Plots of the YEZ-2A Full Scale Hull-only CFD Model for Each Angle of Attack from the Longitudinal View 

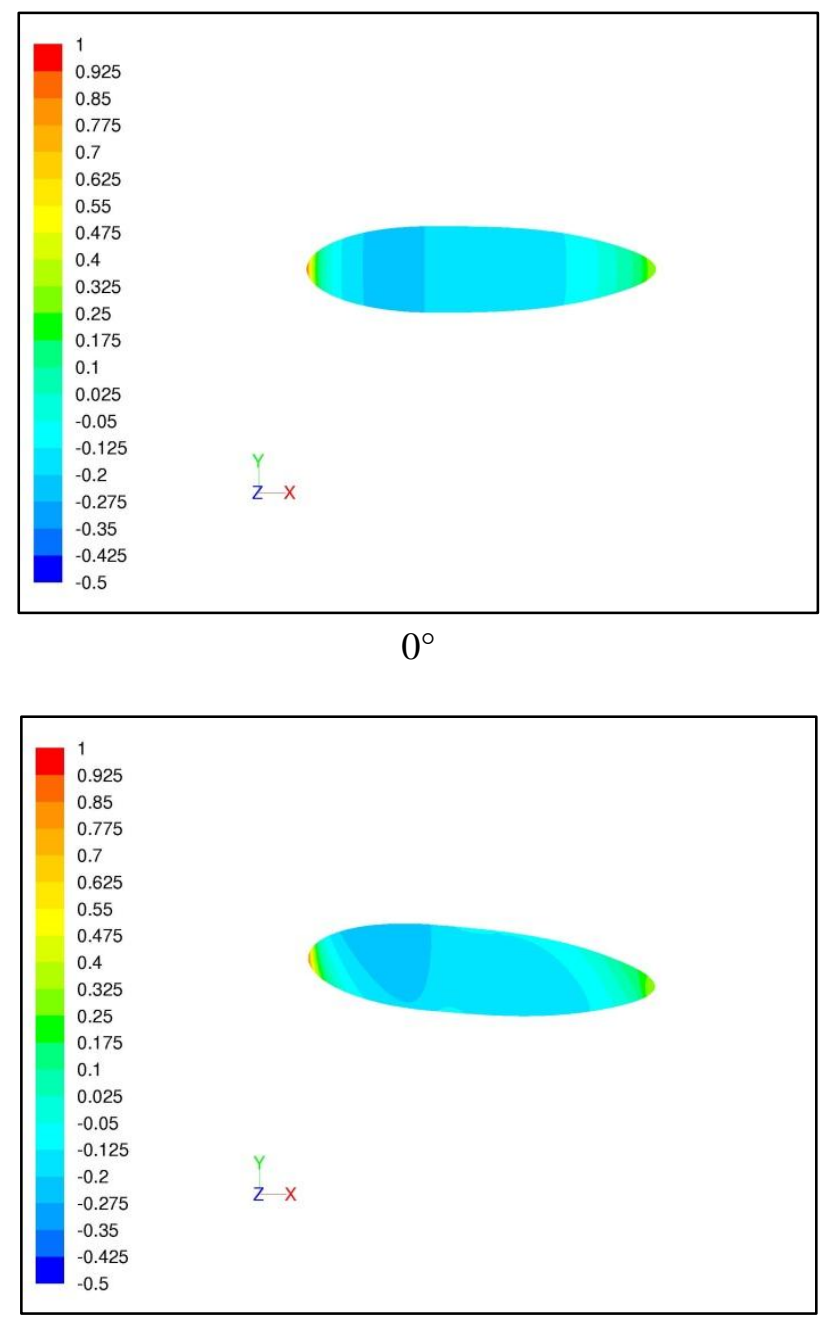

$5^{\circ}$

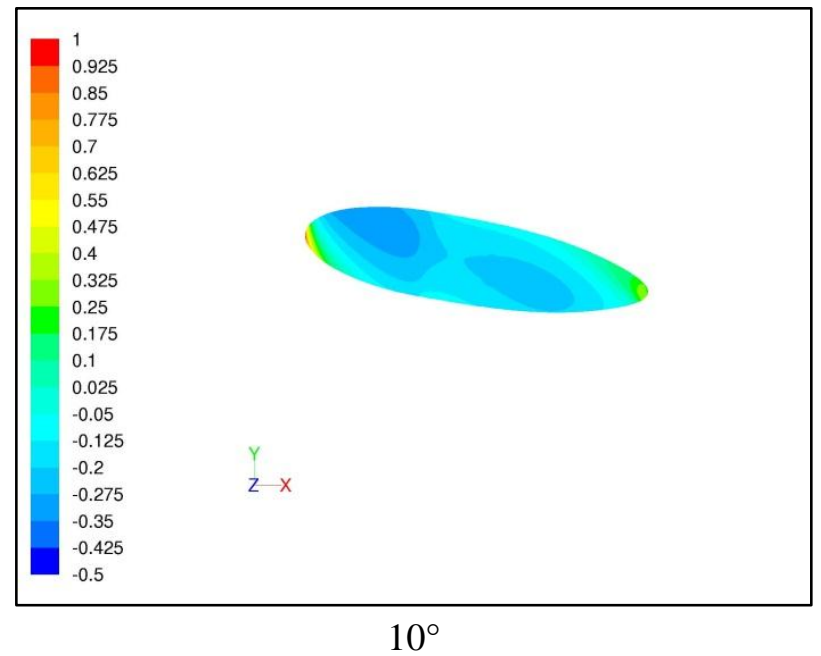

Figure B-4: Pressure Coefficient Contour Plots of the YEZ-2A Full Scale Hull-only CFD Model for Each Angle of Attack from the Longitudinal View 

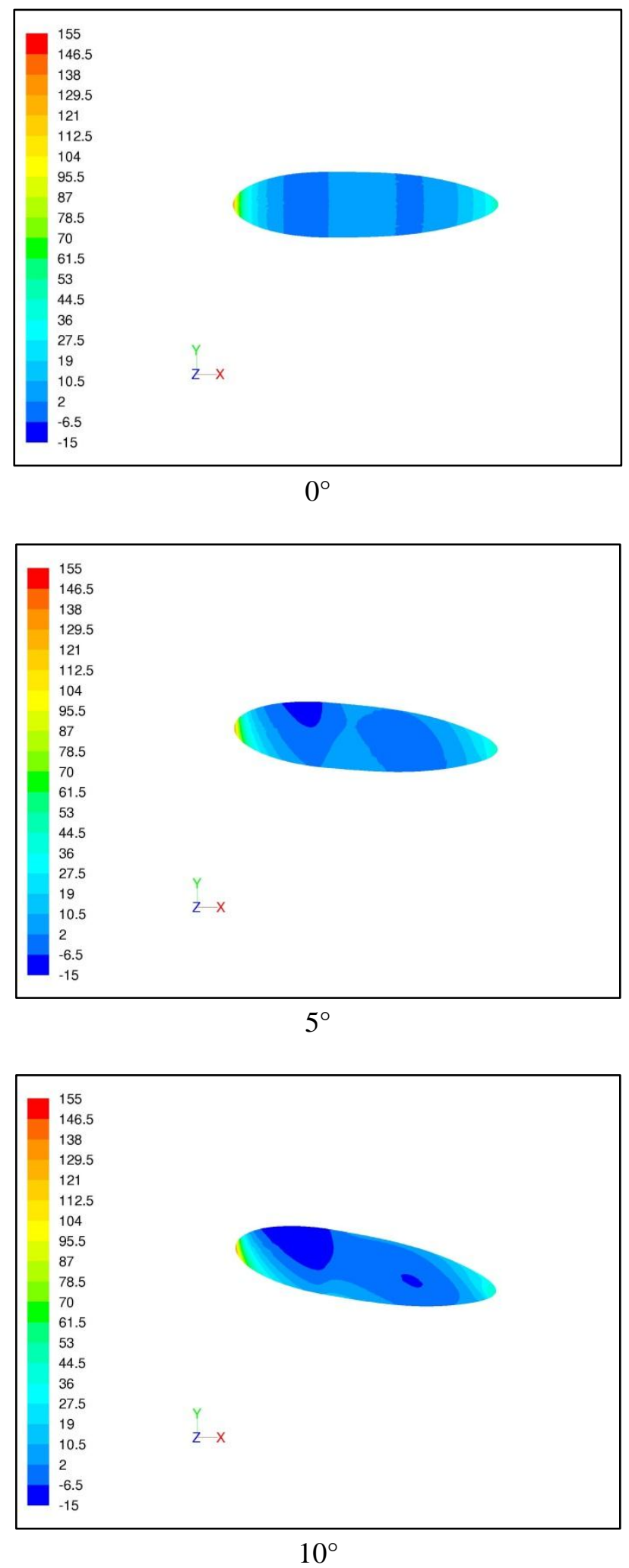

Figure B-5: Total Pressure (pascal) Contour Plots of the YEZ-2A Full Scale Hull-only CFD Model for Each Angle of Attack from the Longitudinal View 

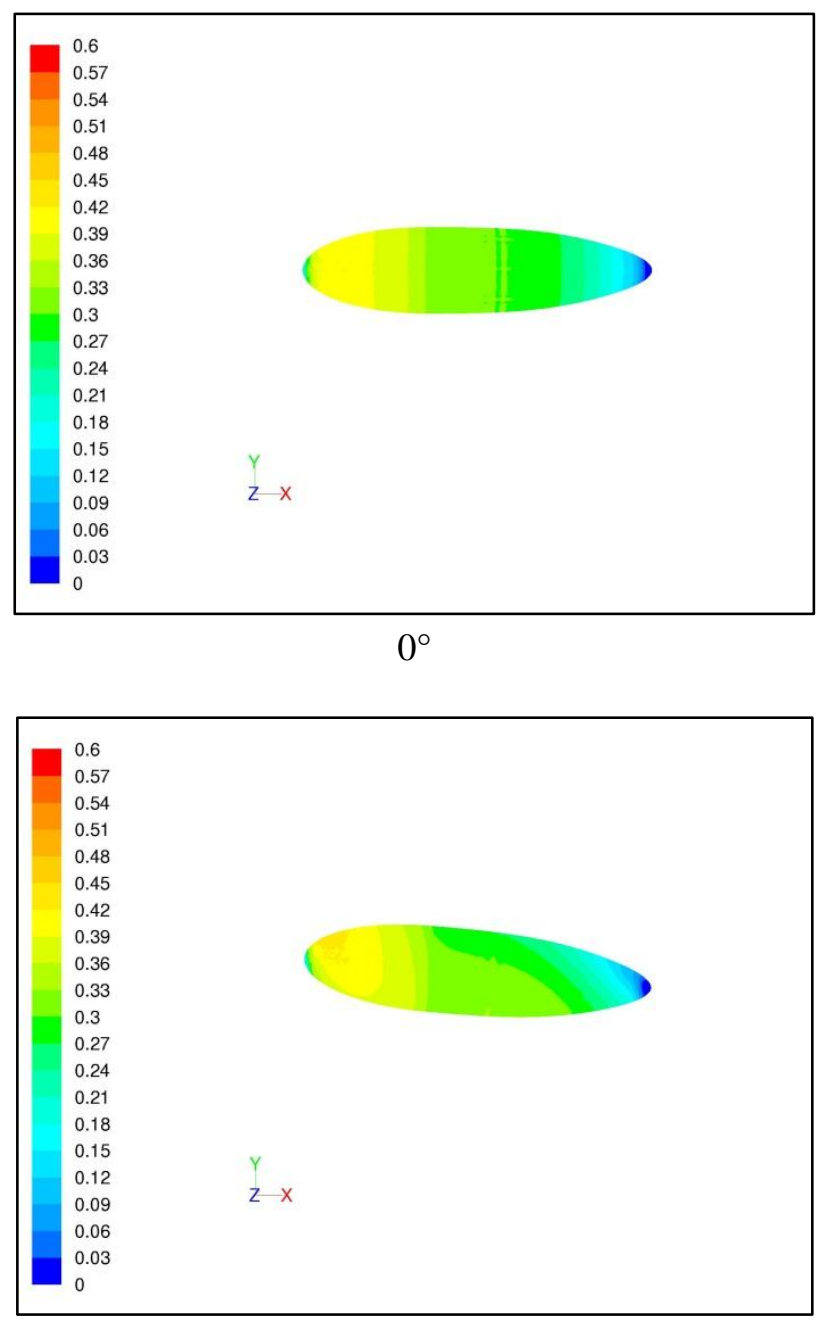

$5^{\circ}$

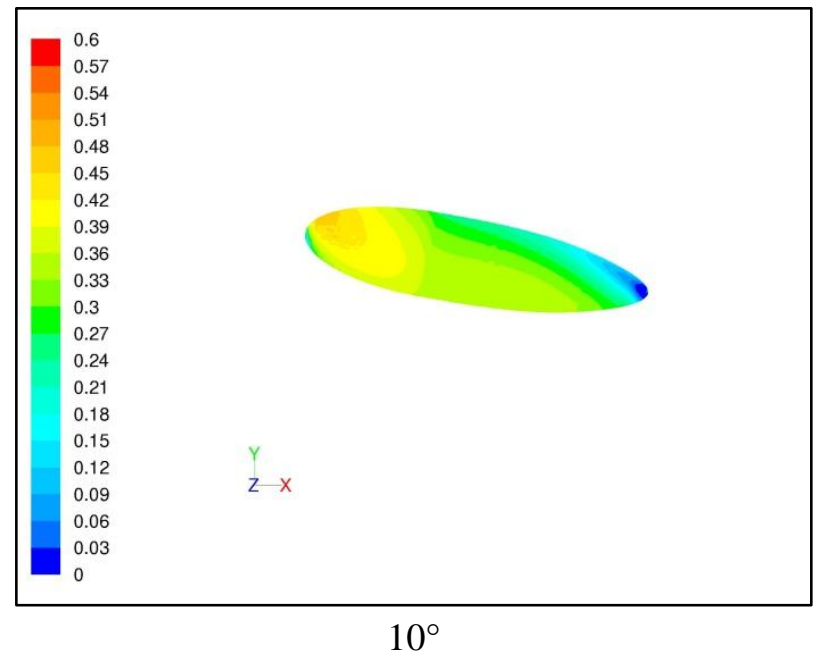

Figure B-6: Shear Stress (pascal) Contour Plots of the YEZ-2A Full Scale Hull-only CFD Model for Each Angle of Attack from the Longitudinal View 

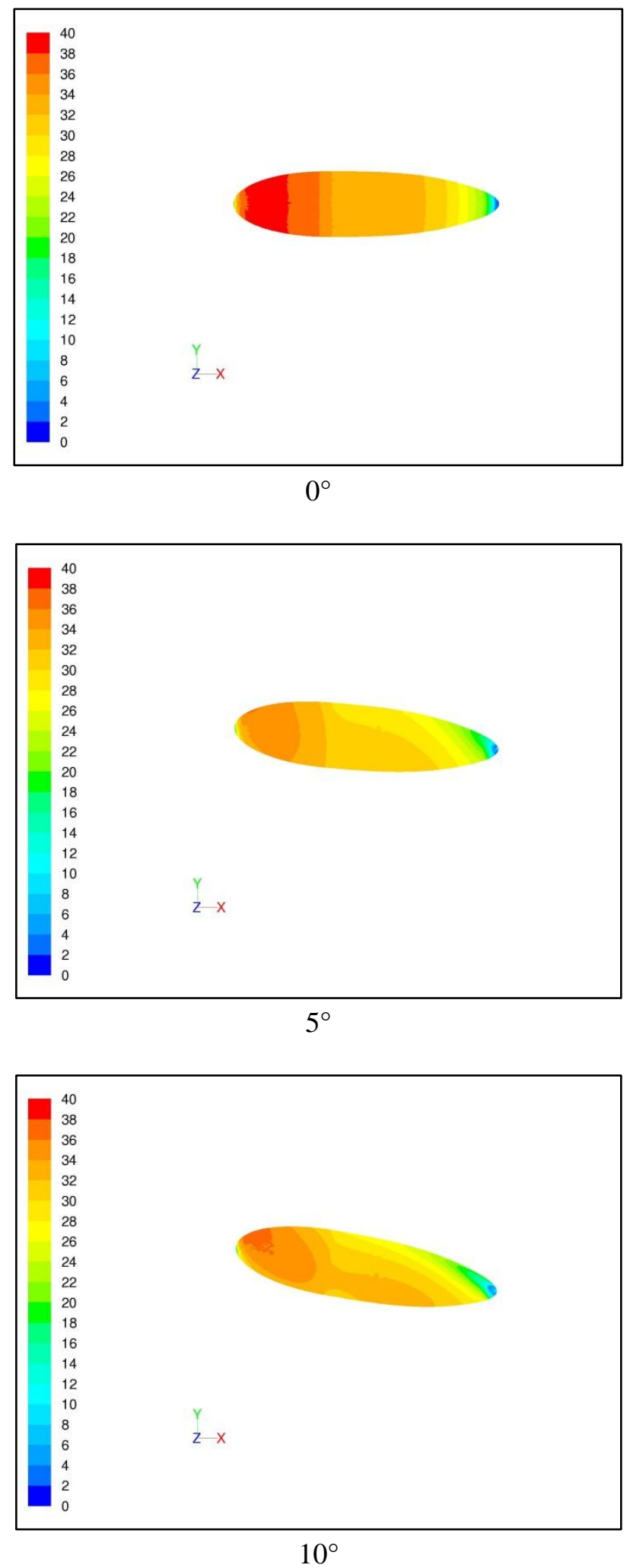

Figure B-7: y+ Contour Plots of the YEZ-2A Full Scale Hull-only CFD Model for Each Angle of Attack from the Longitudinal View 


\section{Appendix C Axisymmetric Investigations}

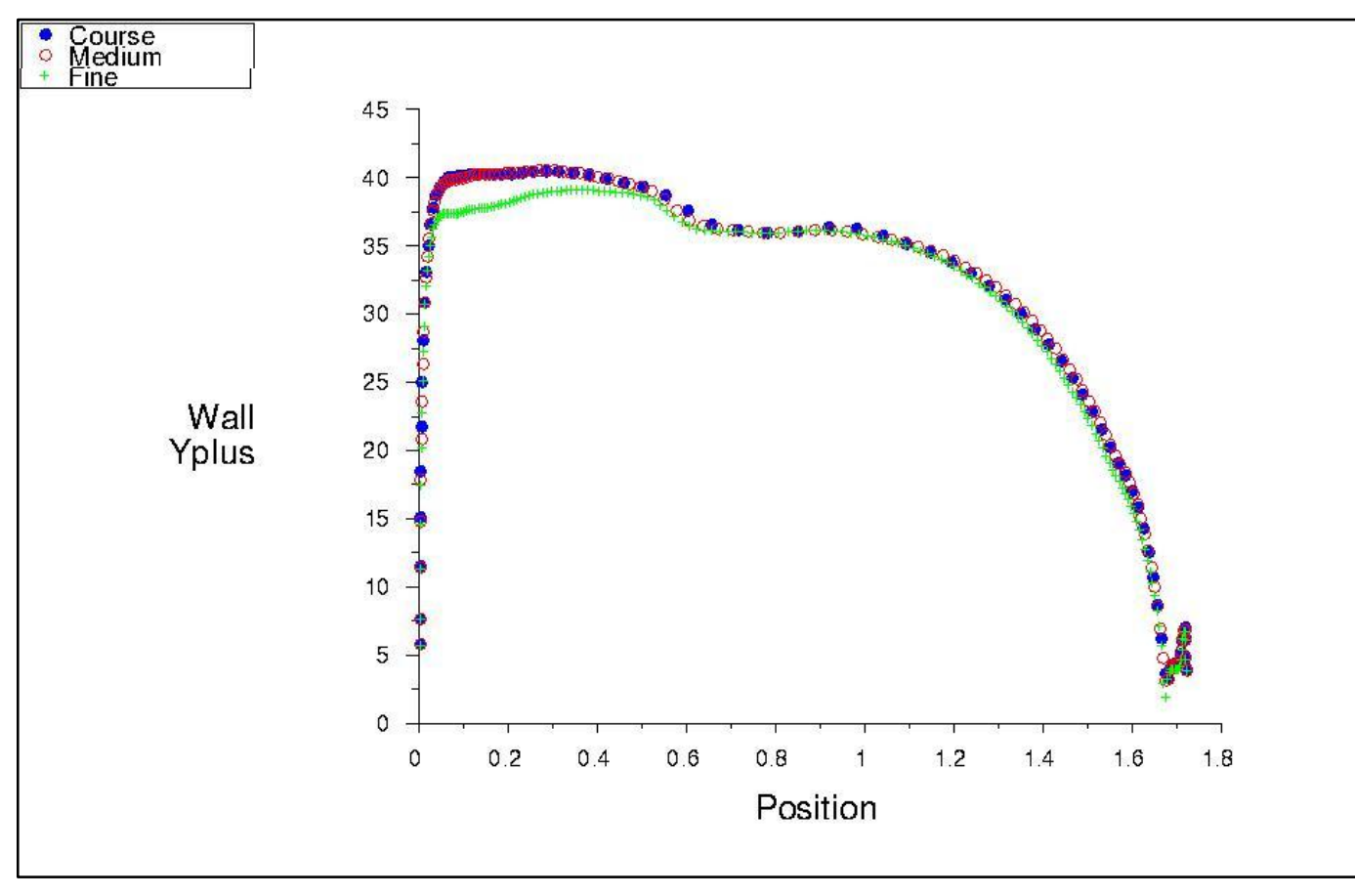

Model Scale

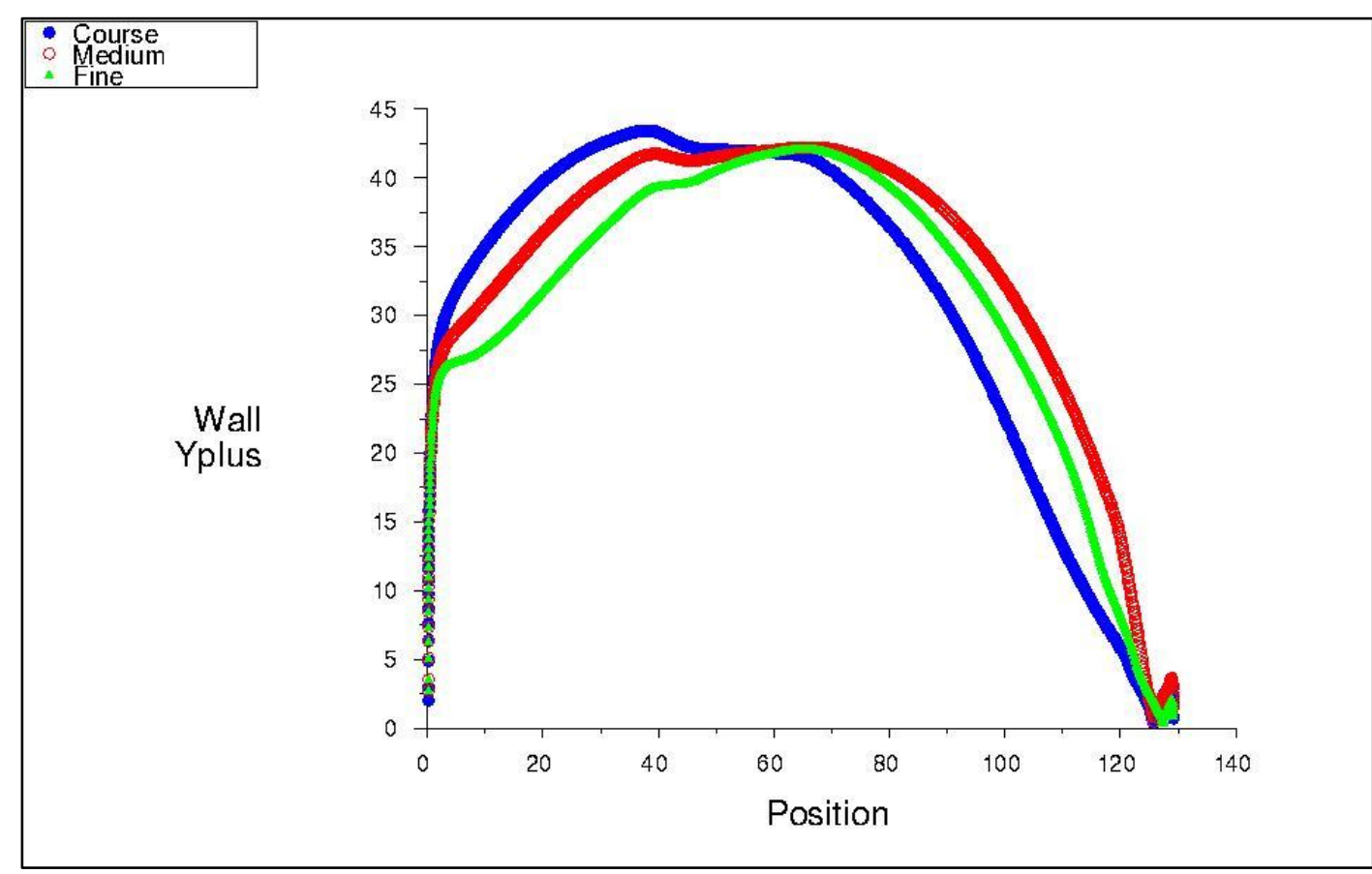

Full Scale

Figure C-1: Wall y+ Values versus Axial Position (m) for Each Model and Grid Resolution 


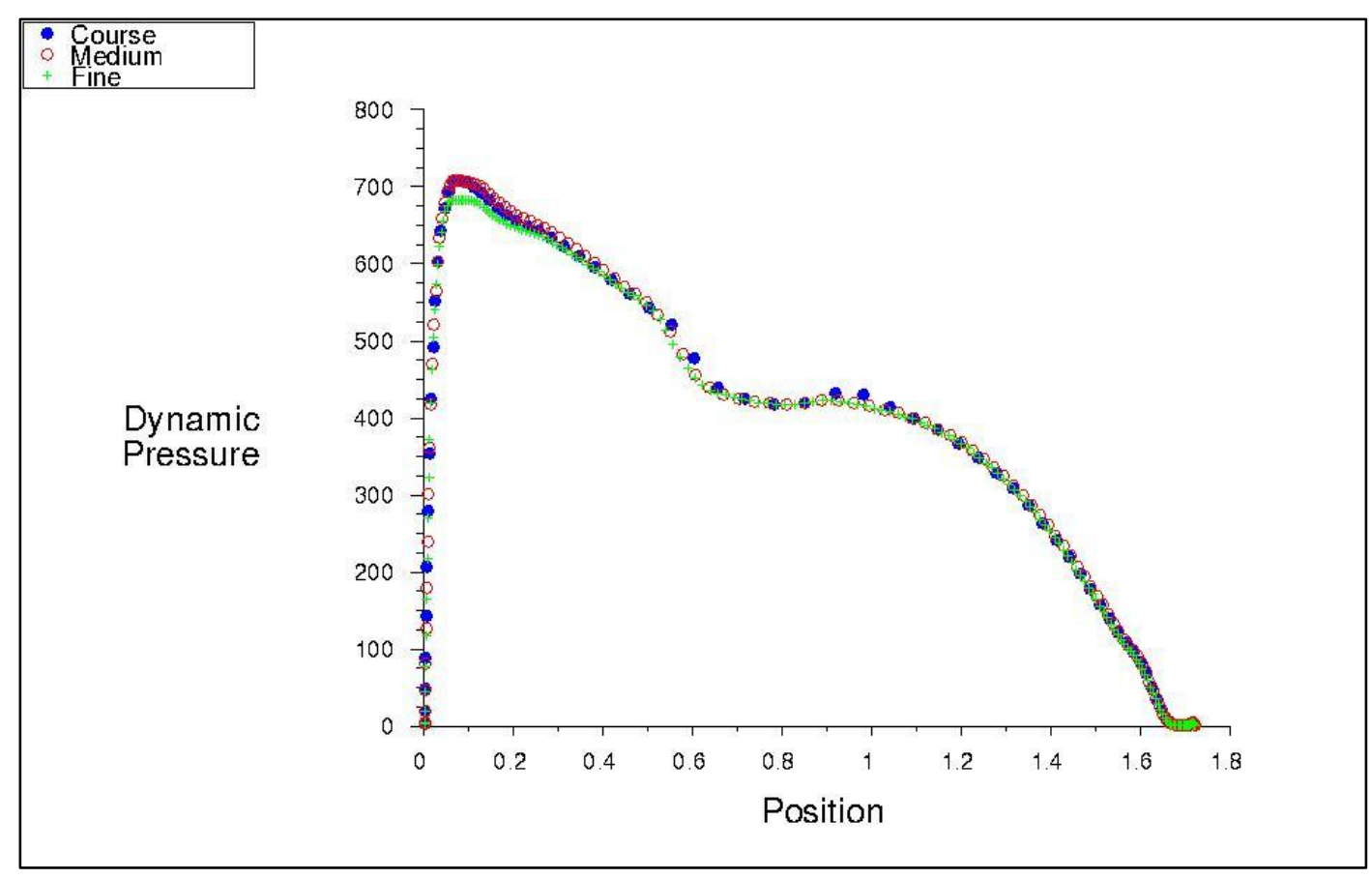

Model Scale

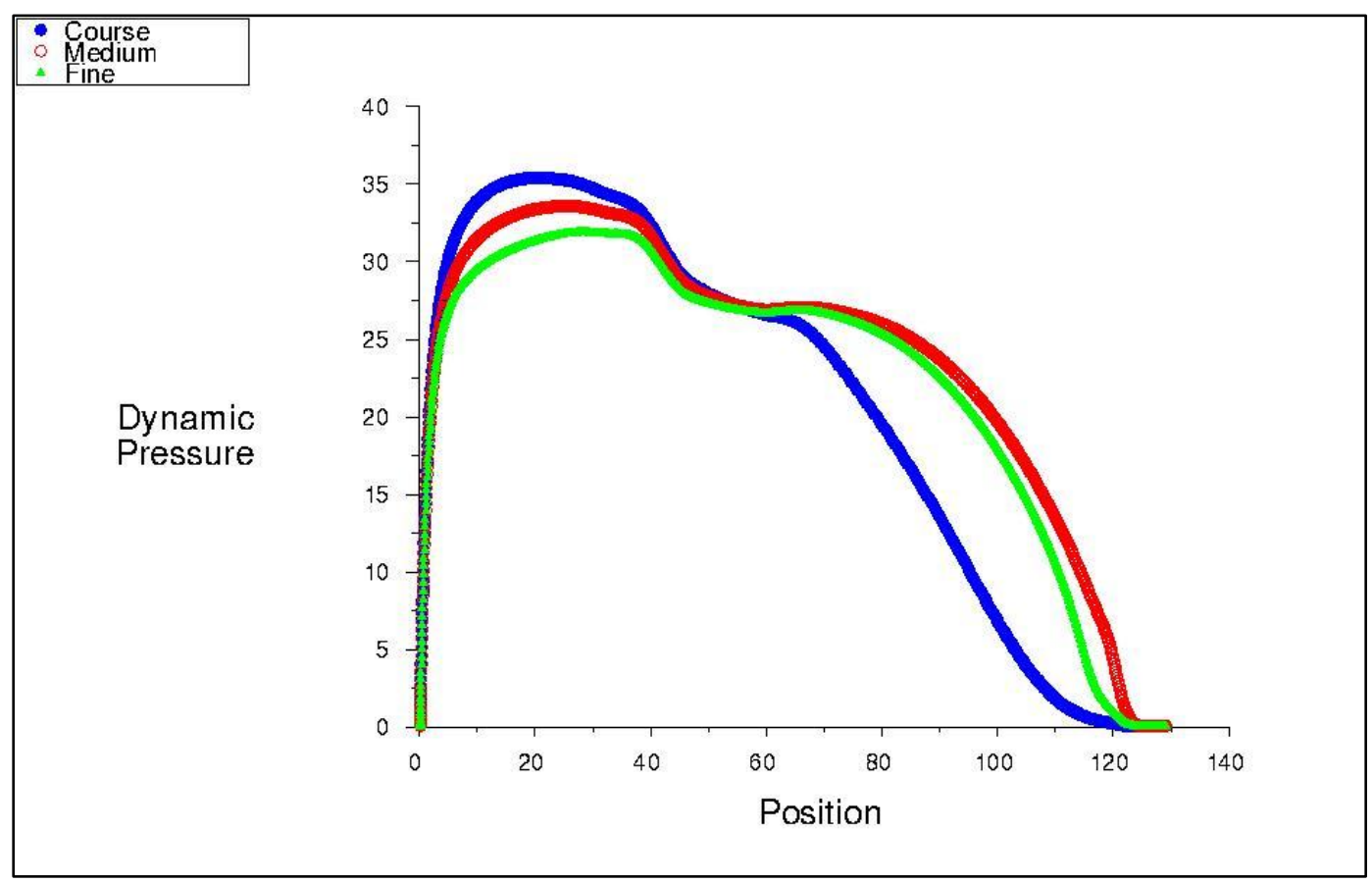

Full Scale

Figure C-2: Dynamic Pressure (pascal) versus Axial Position (m) for Each Model and Grid Resolution 


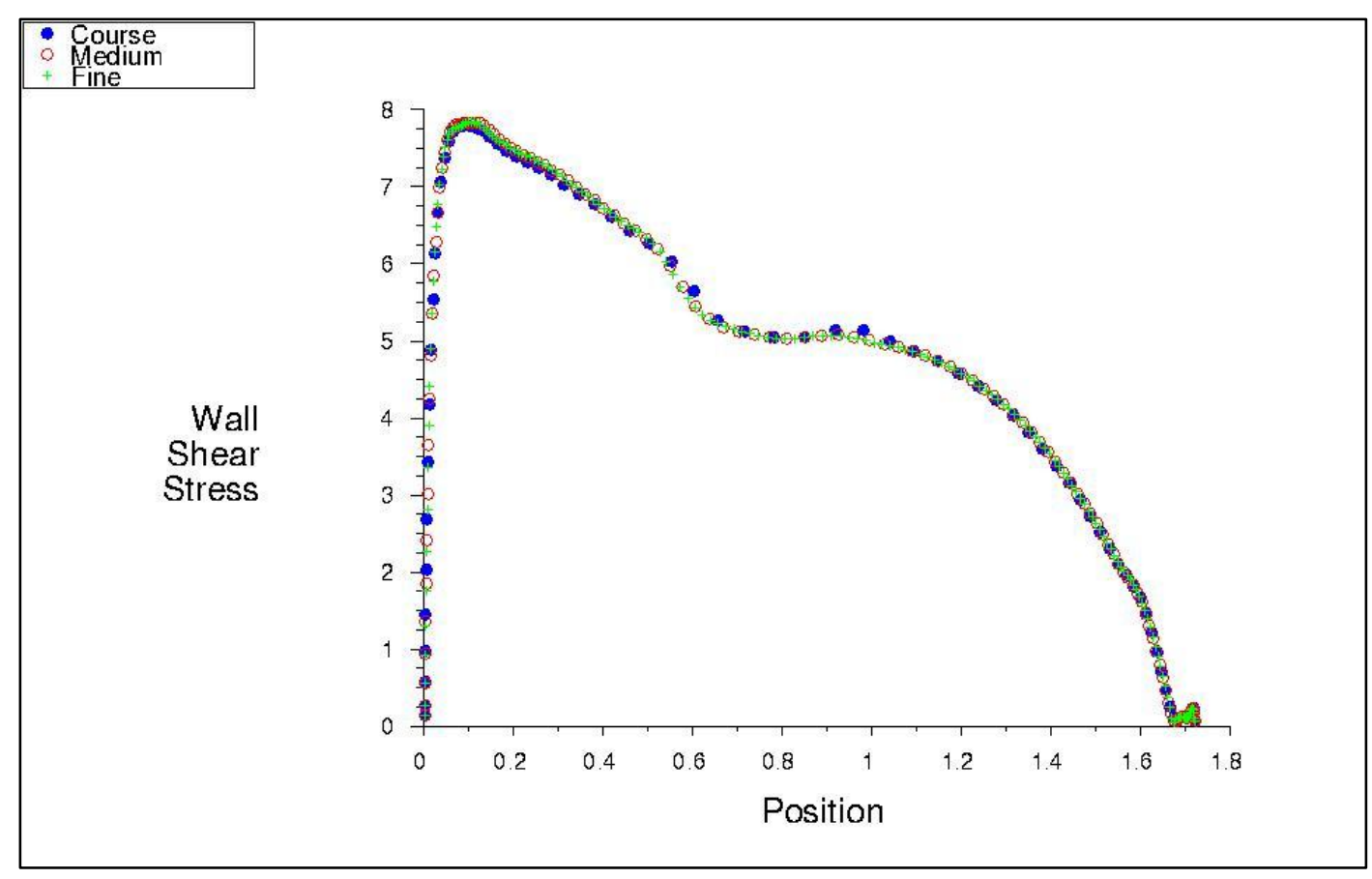

Model Scale

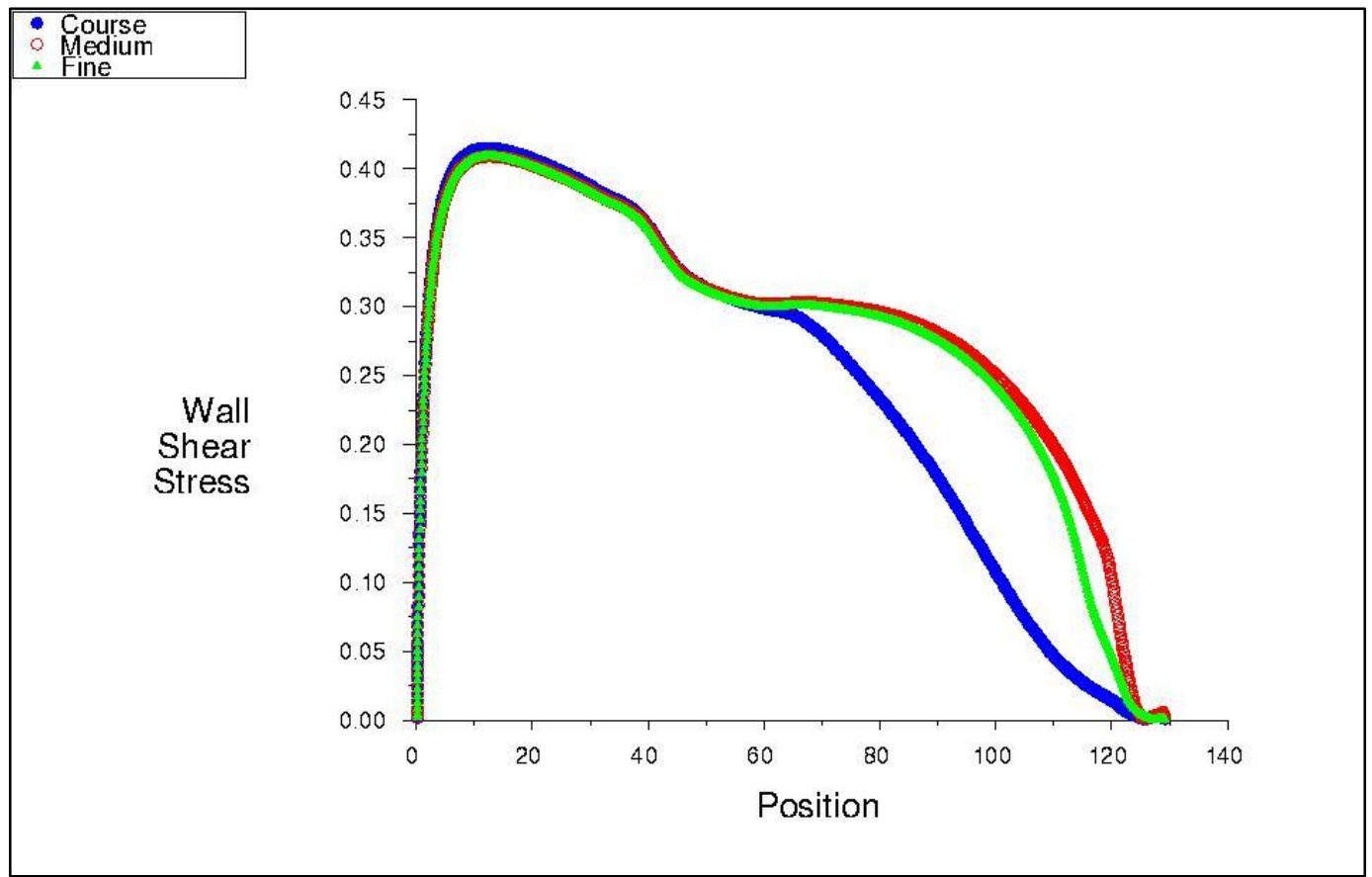

Full Scale

Figure C-3: Wall Shear Stress (pascal) versus Axial Position (m) for Each Model and Grid Resolution 


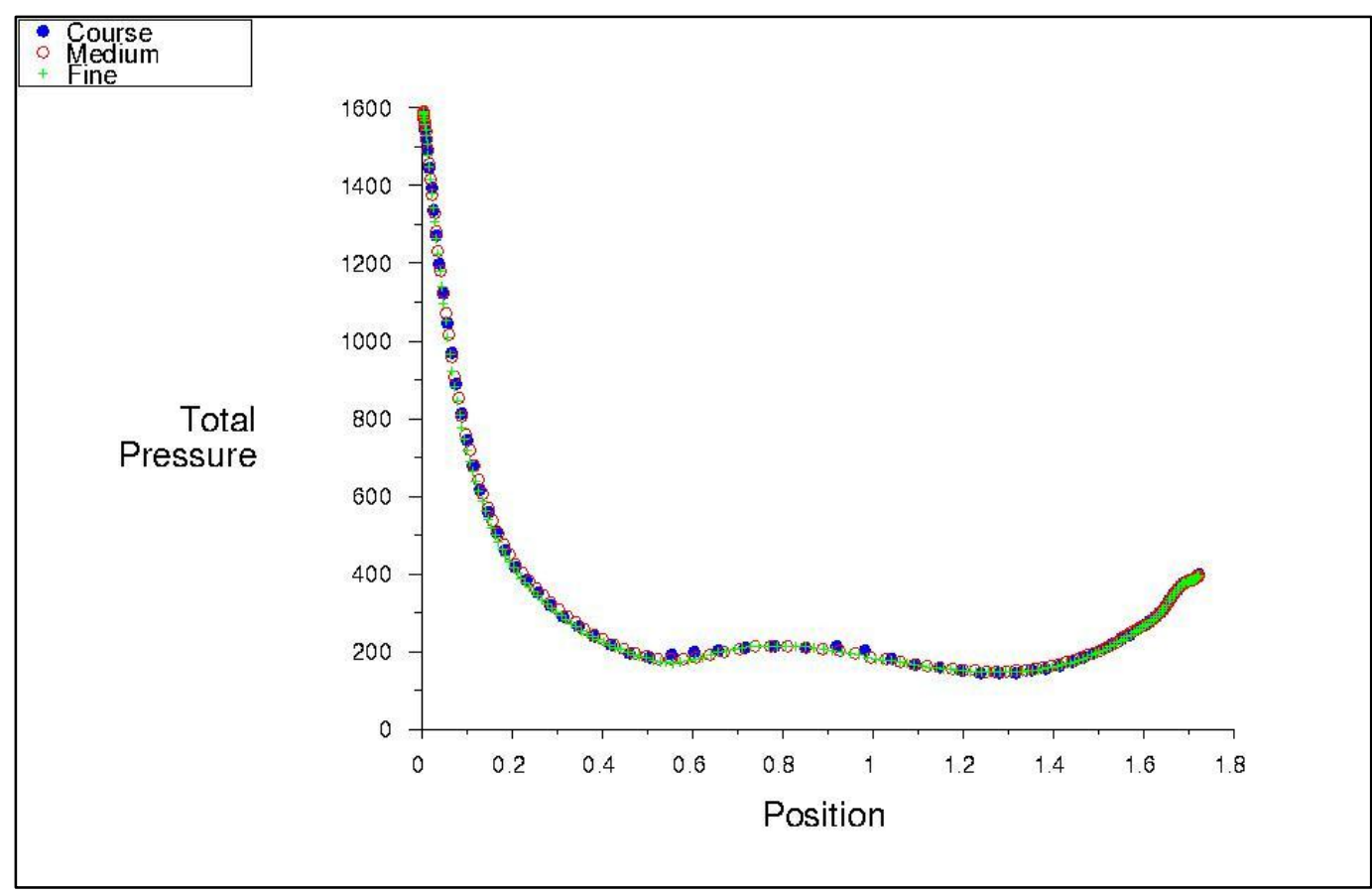

Model Scale

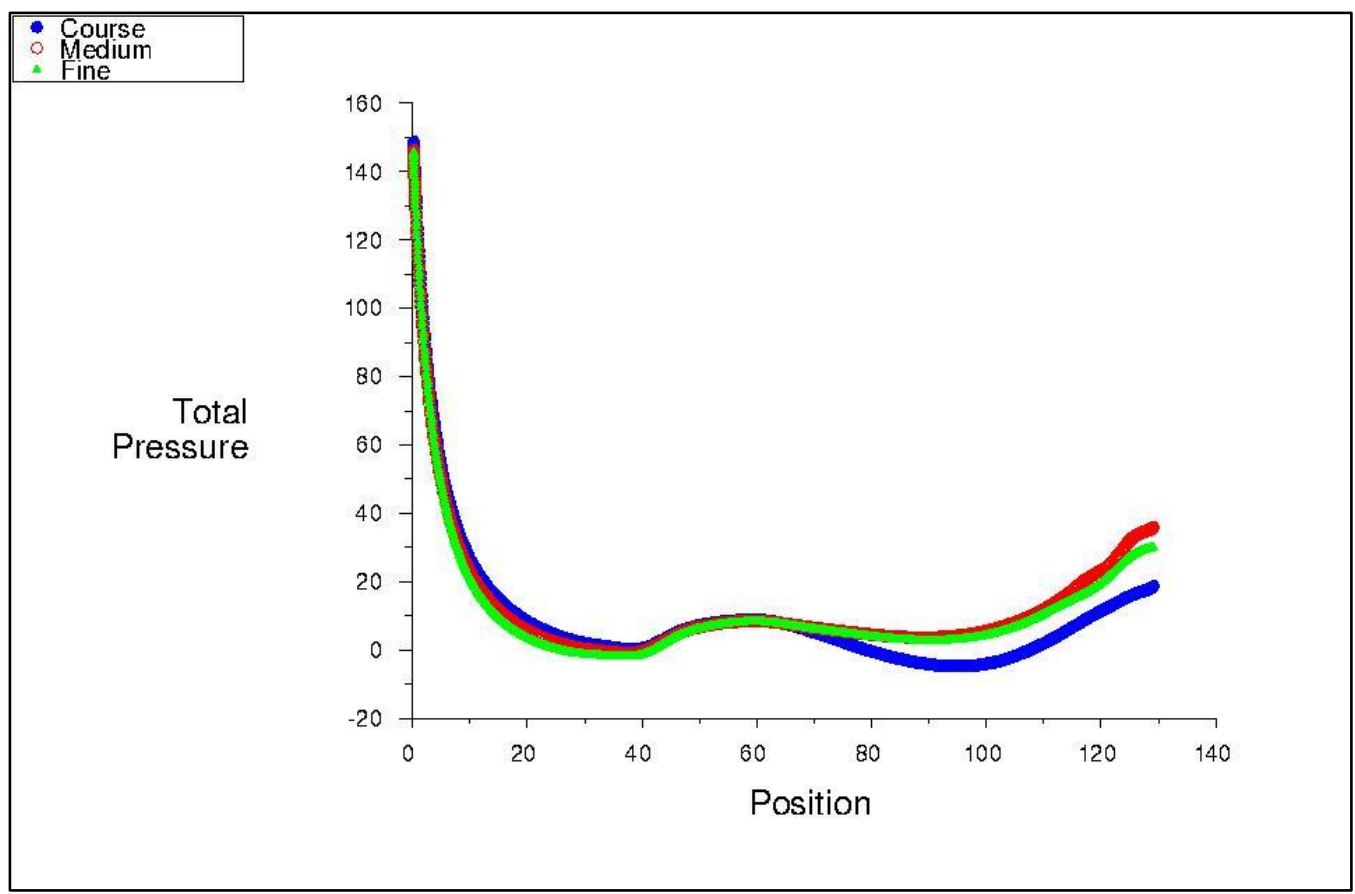

Full Scale

Figure C-4: Total Pressure (pascal) versus Axial Position (m) for Each Model and Grid Resolution 


\section{Transient Setup}

\section{1:75 Scale}

$$
V_{\text {max }}=33.825 \frac{\mathrm{m}}{\mathrm{s}}
$$

$\Delta x=0.01433 m$

$$
\Delta t \approx \frac{\Delta x}{V}=0.000423 \mathrm{~s}
$$

Initialized with 1000 1st order steady state iterations

Original $\Delta t$ set to $0.0004 \mathrm{~s}$

$$
\begin{aligned}
& \frac{\text { iterations }}{\text { time step }}=40 \\
& \text { time steps }=1200 \\
& \text { flow time }=0.48 \mathrm{~s}
\end{aligned}
$$

\section{Full Scale}

$$
\begin{gathered}
V_{\text {max }}=7.689 \frac{\mathrm{m}}{\mathrm{s}} \\
\Delta x=0.10365 \mathrm{~m} \\
\Delta t \approx \frac{\Delta x}{V}=0.01348 \mathrm{~s}
\end{gathered}
$$

Initialized with 3000 1st order steady state iterations, soln controls relaxed $20 \%$

Original $\Delta t$ set to $0.0135 \mathrm{~s}$

$$
\begin{aligned}
& \frac{\text { iterations }}{\text { time step }}=50 \\
& \text { time steps }=2000 \\
& \text { flow time }=27 \mathrm{~s}
\end{aligned}
$$

\begin{tabular}{|r|r|r|r|r|r|r|}
\hline & \multicolumn{3}{|c|}{ Model Scale } & \multicolumn{3}{c|}{ Full Scale } \\
\hline$\Delta \mathrm{t}(\mathrm{s})$ & 0.00004 & 0.0004 & 0.004 & 0.00135 & 0.0135 & 0.135 \\
\hline Flow Time (s) & 0.2 & 0.48 & 0.4 & 10.7 & 27 & 27 \\
\hline Final $C_{D}$ & 0.022834 & 0.022834 & 0.022834 & 0.014208 & 0.014305 & 0.014305 \\
\hline Time Averaged $C_{D}$ & 0.022887 & 0.022856 & 0.022861 & 0.013384 & 0.013935 & 0.013941 \\
\hline
\end{tabular}

Figure C-5: (Top) Calculation of Original Time Step for Transient Analysis and (Below) Drag Coefficient Results for all Time Steps 


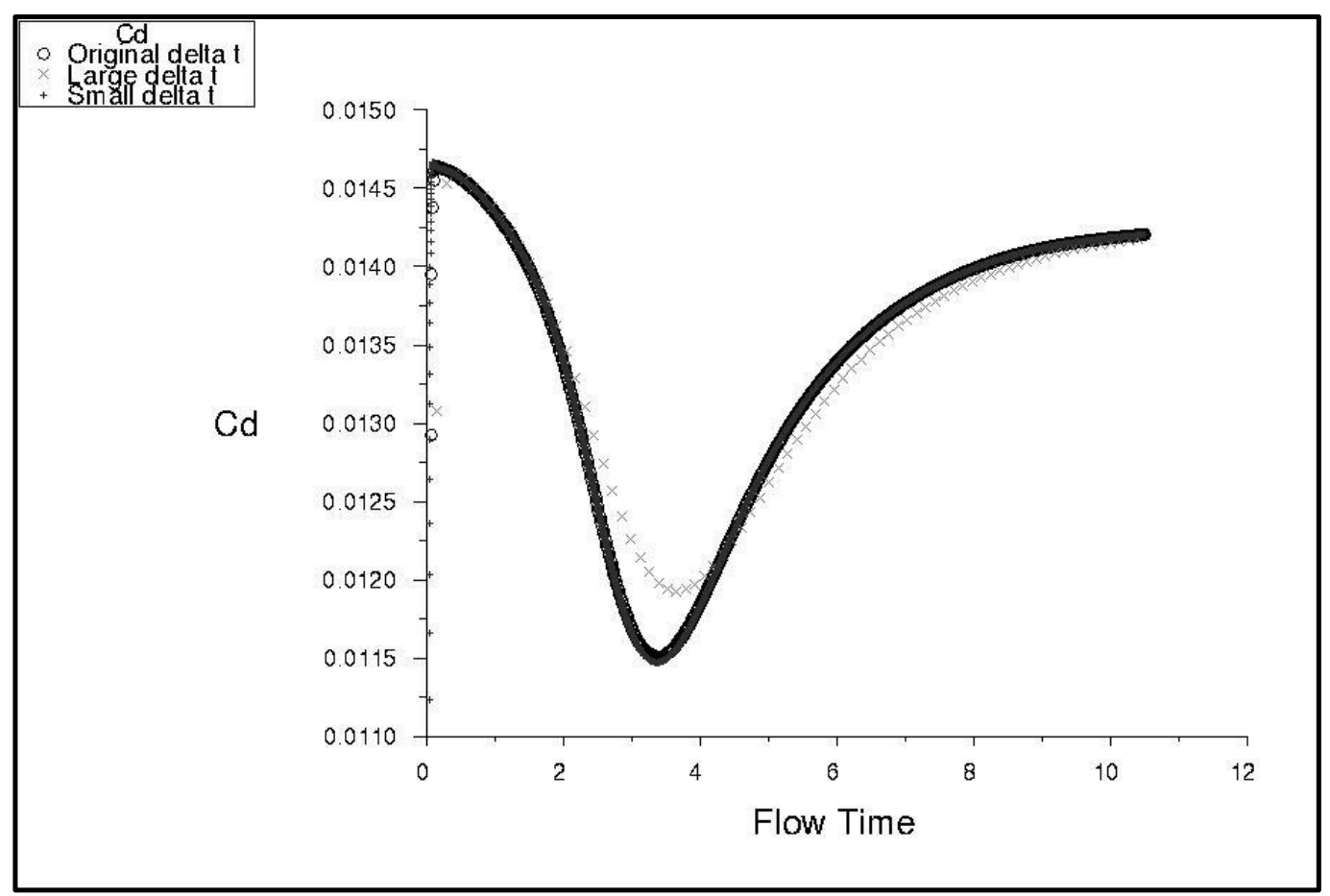

Full Scale: Small $\Delta \mathrm{t}=0.00135 \mathrm{~s}$, Original $\Delta \mathrm{t}=0.0135 \mathrm{~s}$, Large $\Delta \mathrm{t}=0.135 \mathrm{~s}$

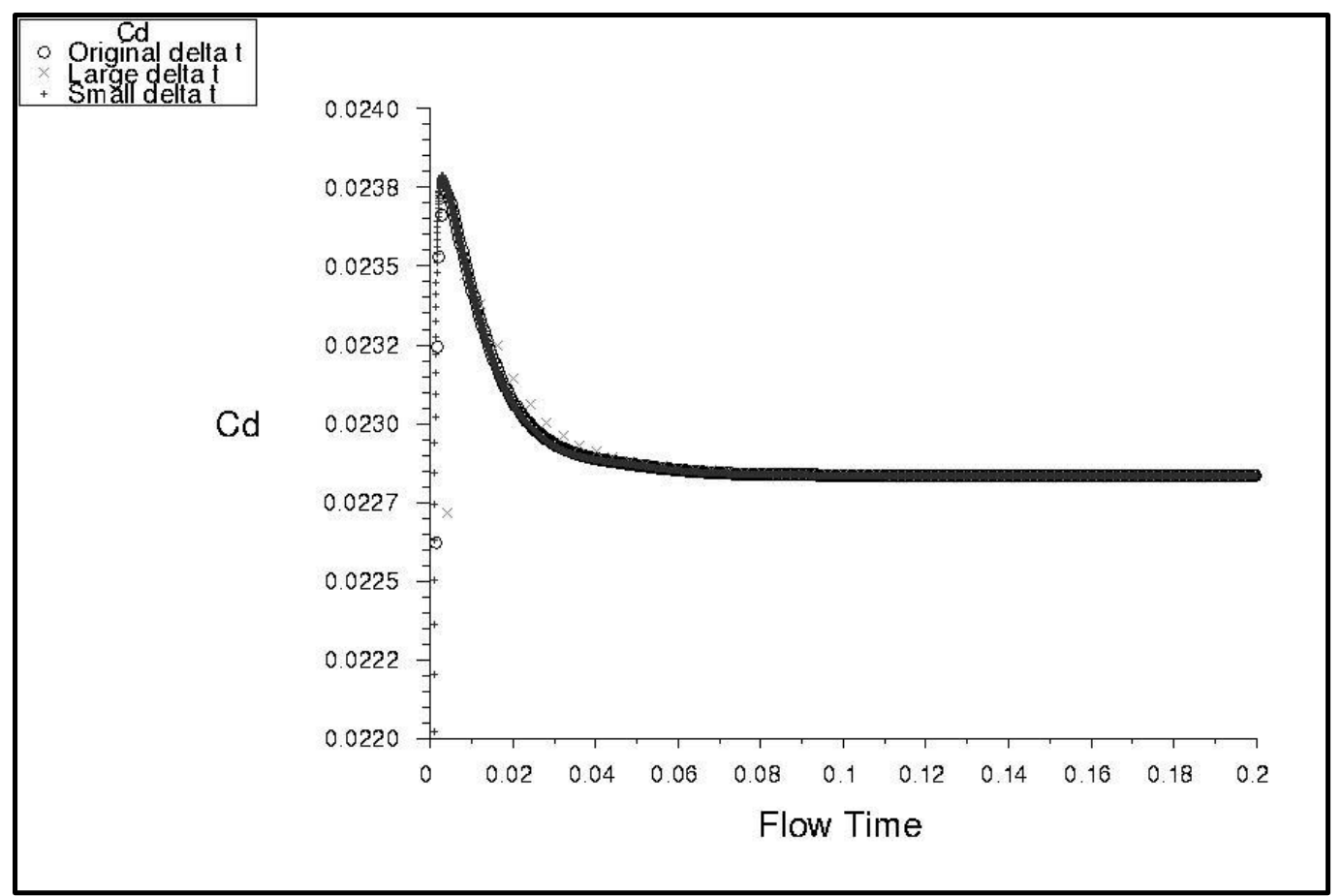

Model Scale, Small $\Delta \mathrm{t}=0.00004 \mathrm{~s}$, Original $\Delta \mathrm{t}=0.0004 \mathrm{~s}$, Large $\Delta \mathrm{t}=0.004 \mathrm{~s}$

Figure C-6: Full Scale and Model Scale Transient Drag Coefficient for Each Time Step

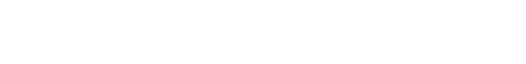

